\title{
The Standard Model quiver in de Sitter string compactifications
}

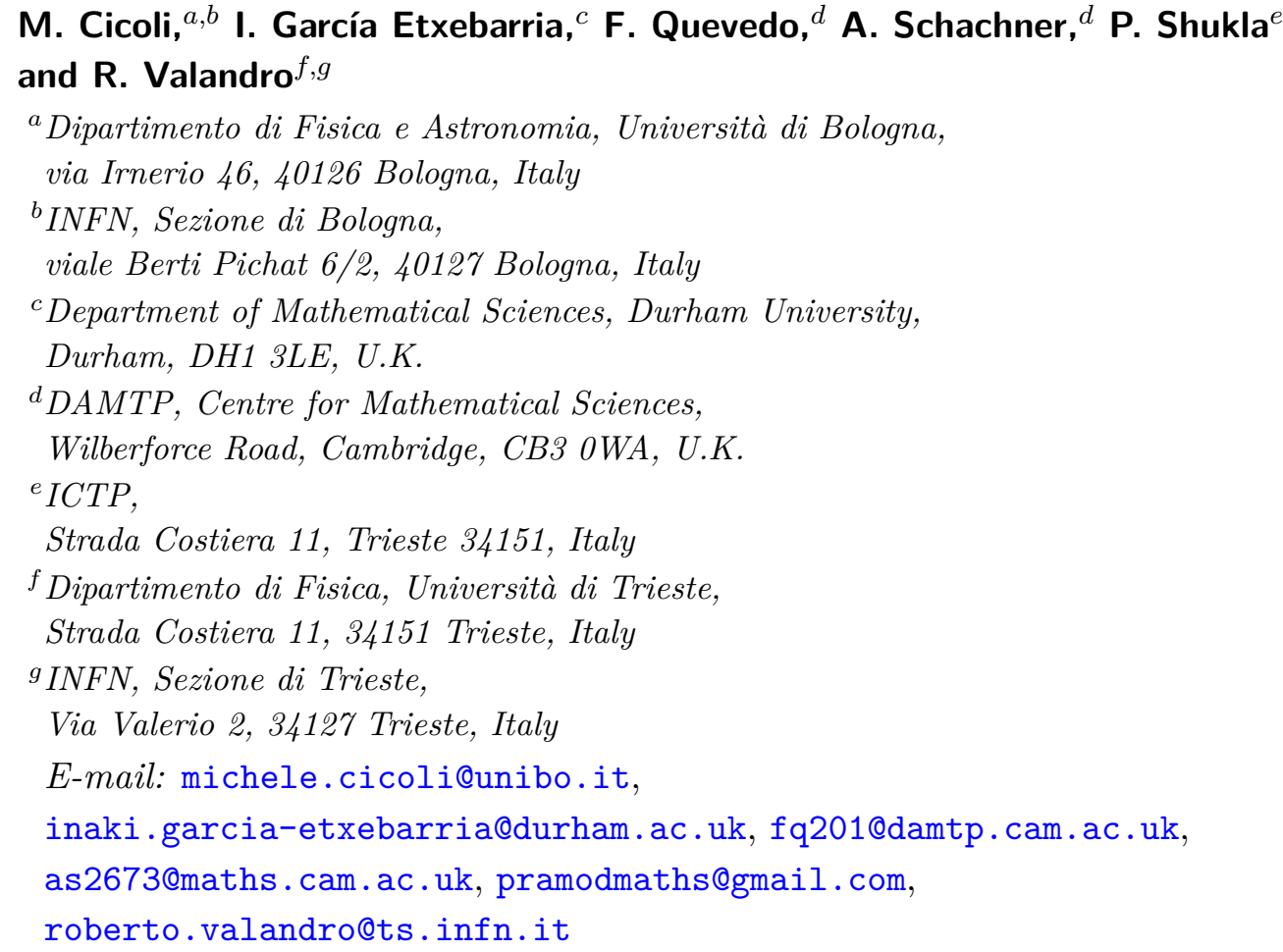

ABstract: We argue that the Standard Model quiver can be embedded into compact Calabi-Yau geometries through orientifolded D3-branes at del Pezzo singularities $\mathrm{dP}_{n}$ with $n \geq 5$ in a framework including moduli stabilisation. To illustrate our approach, we explicitly construct a local $\mathrm{dP}_{5}$ model via a combination of Higgsing and orientifolding. This procedure reduces the original $\mathrm{dP}_{5}$ quiver gauge theory to the Left-Right symmetric model with three families of quarks and leptons as well as a Higgs sector to further break the symmetries to the Standard Model gauge group. We embed this local model in a globally consistent Calabi-Yau flux compactification with tadpole and Freed-Witten anomaly cancellations. The model features closed string moduli stabilisation with a de Sitter minimum from T-branes, supersymmetry broken by the Kähler moduli, and the MSSM as the low energy spectrum. We further discuss phenomenological and cosmological implications of this construction.

Keywords: D-branes, Flux compactifications, Superstring Vacua

ArXiv EPrint: 2106.11964 


\section{Contents}

1 Introduction 1

2 The Standard Model quiver and orientifolded $\mathrm{dP}_{n}$ singularities $\quad 4$

2.1 Calabi-Yau singularities and del Pezzo surfaces 4

2.2 Large volume perspective of D3-branes at singularities 5

2.3 Why branes at singularities? 8

2.4 The $\mathrm{dP}_{5}$-quiver and its involutions 9

2.5 Higgsing the $\mathrm{dP}_{5}$ quiver gauge theory — model I 14

$\begin{array}{lll}2.6 & \text { Matter spectrum for model I } & 19\end{array}$

$2.7 \quad F$ - and $D$-term flatness 22

3 Calabi-Yau threefolds with diagonal $\mathrm{dP}_{5}$ divisors $\quad \mathbf{2 4}$

3.1 Embedding the local model in a compact CY threefold 24

$\begin{array}{lll}3.2 & \text { Calabi-Yau threefolds with diagonal } \mathrm{dP}_{n} & 25\end{array}$

4 Global embedding of $\mathrm{dP}_{5}$ model $\quad 26$

$\begin{array}{lll}4.1 \text { Geometric data } & 26\end{array}$

4.2 Orientifold involution 28

4.3 Embedding of the local model 28

$\begin{array}{lll}4.4 & \text { Non-perturbative effects } & 29\end{array}$

$\begin{array}{lll}4.5 & \text { D-brane setup } & 30\end{array}$

5 Moduli stabilisation $\quad \mathbf{3 5}$

$\begin{array}{ll}\text { 5.1 Background fluxes and D-terms } & 36\end{array}$

$\begin{array}{lll}5.2 & \text { Non-perturbative and } \alpha^{\prime} \text { effects } & 38\end{array}$

5.3 Choices of underlying parameters 41

6 Phenomenological and cosmological implications 43

$\begin{array}{lll}7 & \text { Conclusions } & 46\end{array}$

A Analysing the CY threefolds from the KS database 49

A.1 Diagonal dP divisors in CY hypersurfaces in toric ambient spaces 49

$\begin{array}{ll}\text { A.2 Scanning results for (diagonal) del Pezzo divisors } & 50\end{array}$

B CICY threefolds with diagonal $\mathrm{dP}_{\mathbf{5}}$ divisors $\quad \mathbf{5 3}$

B.1 $\mathrm{dP}_{5}$ embedded in a CICY with $h^{1,1}=2 \quad 53$

B.2 CICY examples with $h^{1,1}=3 \quad 54$

B.3 A CICY example with $h^{1,1}=4 \quad 57$ 
C.1 D-terms for $\mathrm{SU}(N)$ and $\mathrm{USp}(2 N)$ gauge groups 58

$\begin{array}{lll}\text { C.2 Larger model I } & 59\end{array}$

C.3 Higgsing model II 62

D Line orientifold of the complex cone over $\mathrm{dP}_{5} \quad 65$

\section{Introduction}

The main argument for the study of string theory remains its potential to explain all natural phenomena, including gravity, within a consistent quantum framework. In this sense, it is usually stated that string theory provides the UV completion of the Standard Model. However, despite many efforts for the past 30 years, it is fair to say that there is not yet a consistent string construction that includes the Standard Model and does not have some unrealistic features.

The search for realistic string models seems hopeless due to the huge degeneracy of string compactifications. This is usually compared with the proverbial search for a needle in a haystack. Similar to using a magnet to find the needle we can follow a bottom-up modular approach in the search for a realistic string model [1]. We may split the search into at least three independent challenges:

- Search for local string constructions in terms of intersecting branes [1-7] or local Ftheory models [8-11] that includes the chiral matter of the Standard Model in which gravity is decoupled.

- Search for string mechanisms in which global issues such as moduli stabilisation, supersymmetry breaking, inflation or alternatives are addressed, ignoring the potential realisation of the Standard Model particles and interactions beyond gravity.

- Once a successful framework for each of the two challenges above has been found, combine both constructions to incorporate the Standard Model in a fully-fledged string compactification.

Each of these steps is a major challenge by itself but the approach is much simpler and systematic than direct top-down searches for realistic string models.

The last decade has brought enormous advances in our ability to construct semirealistic vacua in the framework of type IIB compactifications. Much of the focus has been on F-theory model building (starting with [8-11]), and has resulted in a rich set of models, with a number of promising features when it comes to model building (see for instance [12] and references therein).

One of the defining characteristics of F-theory model building is the description of sectors where the string coupling constant $g_{s}$ becomes large. It is possible to understand 
such regimes using duality with M-theory. However, since our knowledge of the behaviour of M-theory on highly curved manifolds is rather limited, our understanding of F-theory models is generically limited to features that can be continued in a supersymmetric way to weakly curved backgrounds. Furthermore, moduli stabilisation is not included in these constructions limiting their potential contact with the real world.

In this paper we focus instead on the complementary regime of type IIB models which contain highly curved - singular, in fact - regions. More concretely, we will explore the case of D3-branes at del Pezzo singularities in global type IIB Calabi-Yau (CY) compactifications. Branes probing singularities lead to interesting low energy dynamics, which can be understood at sufficiently small string coupling $g_{s}$. The visible sector arising from the modes at the singularity is described by a collection of fractional branes which are conveniently represented as nodes in 2-dimensional graphs referred to as quiver diagrams. Open strings stretched between stacks of fractional branes give rise to a massless spectrum of matter fields in bi-fundamental representations joining the various quiver nodes via directed lines.

Many of the required tools for studying the singular regions are familiar from various previous analyses of branes at IIB singularities with orientifolds; our contribution is the construction of explicit global models that include singularities relevant to realistic model building, and the detailed analysis of their features. The constructions provided in this paper reproduce the MSSM exactly in stark contrast to previous local [13-17] and global [18-22] investigations. We will find that reproducing the SM spectrum does not require flavour D7-branes. In spite of the local nature of the model, it does not imply that one should expect to be able to simply "glue" the local physics to any arbitrary compact manifold since satisfying the multiple phenomenological constraints on the model proves to be very stringent. In fact, after extensive searches for candidates, we only found a few models which possess realistic features both at the local and global level.

In order to find a suitable local realisation of the Standard Model, we have been guided by the seminal work by Wijnholt [23], who provided two scenarios to obtaining the Standard Model quiver from a single D3-brane at a $\mathrm{dP}_{5}$ singularity. Essential features of both of these two scenarios are the presence of appropriate orientifold involutions ${ }^{1}$ and intricate Higgsing operations. The resulting quiver in these scenarios is of form given in figure 1. It consists of the Standard Model degrees of freedom together with right-handed neutrinos and an extra $\mathrm{U}(1)_{B}$. The $\mathrm{U}(1)$ on the bottom right is obtained either from identifying two $\mathrm{U}(1)$ 's in a covering quiver or from a larger quiver via Higgsing $\mathrm{SU}(2) \times \mathrm{U}(1) \rightarrow \mathrm{U}(1)$. In fact, the latter scenario corresponds to having a supersymmetric version of the Minimal Left-Right Symmetric Model [27-29] as an intermediate step. The local models studied in this paper will be of this form. It should be noted at this point, though, that there remains a rich structure of largely unexplored but phenomenologically promising models from fractional D3-branes at orientifolded $\mathrm{dP}_{n}$ singularities with $n \geq 5$. In this paper we will restrict ourselves to the $\mathrm{dP}_{5}$ case, but the analysis of cases with higher $n$ would certainly be interesting.

\footnotetext{
${ }^{1}$ This seems to be a general theme: minimal quiver extensions of the supersymmetric Standard Model themselves are often unoriented [24-26].
} 


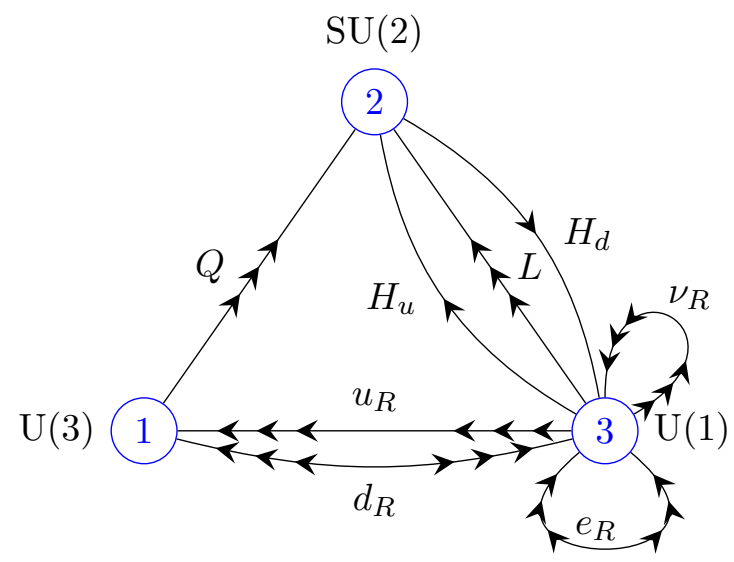

Figure 1. Standard Model quiver containing an additional U(1) $B$. The right-handed neutrinos transform in the (trivial) adjoint of $\mathrm{U}(1)$ and are thus uncharged under all gauge group factors.

In this paper we will go further than a purely local analysis, and provide a seemingly phenomenologically viable global embedding of the orientifolded quiver gauge theory, so that our gauge dynamics is coupled to gravity. The presence of a $\mathrm{dP}_{5}$ singularity is ensured by having a diagonal divisor of $\mathrm{dP}_{5}$ topology inside the CY. The diagonality condition allows to take the singular limit for the $\mathrm{dP}_{5}$ divisor by taking a single linear combination of 2-cycle volumes to zero without shrinking any additional divisors, see for instance [30].

A noteworthy complication in carrying out this program is the absence of diagonal $\mathrm{dP}_{n}$ divisors with $1 \leq n \leq 5$ at Hodge numbers $h^{1,1} \leq 40$ in the Kreuzer-Skarke (KS) database [31]. ${ }^{2}$ We proceed by instead constructing CY threefolds $X$ as complete intersections of two equations in 5-dimensional toric spaces. We show that the global orientifold action on $X$ can be made consistent with the orientifold involution of the local model.

Once we have achieved this global embedding of the local physics, we will devote the rest of the paper to checking that our background is workable and phenomenologically promising. These checks will be developed in detail in the bulk of the paper, but we give a brief summary here. First, since O7/O3-planes carry non-trivial RR-charges, tadpole cancellation requires the presence of further ingredients in the compact space. FreedWitten anomaly cancellation [32] then demands suitable flux backgrounds for various antisymmetric tensors. These fluxes, in turn, affect the $4 \mathrm{D}$ effective theory through non-trivial $F$ - and $D$-terms which stabilise a subset of geometric moduli. Moreover, they can be chosen to give rise to a T-brane background $[33,34]$ which leads to a controlled uplift to $4 \mathrm{D}$ de Sitter minima [35].

Kähler moduli stabilisation necessitates additional non-perturbative effects which arise from wrapping Euclidean D3-branes on internal 4-cycles [36]. At the minimum, supersymmetry is broken spontaneously in the hidden sector by non-vanishing F-terms for bulk Kähler moduli [37]. This breaking is mediated to the visible sector through gravitational interactions. In the absence of sequestering effects for orientifolded quivers [38], soft terms

\footnotetext{
${ }^{2}$ We observed this empirically, but we do not know why it is so.
} 
are of order the gravitino mass which can be either around $10^{10} \mathrm{GeV}$ or at the $\mathrm{TeV}$-scale, depending on the tuning allowed on the flux superpotential. Interestingly, if the gravitino mass is at intermediate scales, our models have all the required features to provide a viable description of the cosmological evolution of our universe, from inflation [39] to the post-reheating epoch [40, 41] involving non-thermal dark matter [42], Affleck-Dine baryogenesis [43] and axionic dark radiation [44-46].

This paper is organised as follows. Section 2 concerns the local model construction from a D3-brane at a $\mathrm{dP}_{5}$ singularity. Subsequently, we highlight several obstacles that appear in obtaining suitable CY threefolds from polytope triangulations in the KS database in section 3. Afterwards, we specialise to a specific complete intersection CY threefold exhibiting the required $\mathrm{dP}_{5}$ singularity. We show that the local model is consistently embedded into the compact CY orientifold background. Further, we provide a fully explicit construction of the D-brane configuration featuring a T-brane background. In section 5, we confirm that closed string moduli can indeed be stabilised in Minkowski or slightly de Sitter minima. We discuss phenomenological implications in section 6 and summarise our conclusions in section 7 .

\section{The Standard Model quiver and orientifolded $\mathrm{dP}_{n}$ singularities}

In this section we will describe in detail the local model giving rise to the Standard Model sector.

\subsection{Calabi-Yau singularities and del Pezzo surfaces}

Let $X$ be a CY threefold. The moduli space $\mathcal{M}_{\mathrm{K}}(X)$ of Kähler classes is characterised by the Kähler cone. Upon approaching the boundary wall of this cone, some parts of $X$ shrink to zero size typically giving rise to singular geometries. This shrinking can happen in various distinct ways as outlined in [47], see also [48-51].

Here, we are particularly interested in the scenario where a single 4-cycle shrinks to a point which was first extensively studied from the string theory point of view in [52,53]. The associated singularities are referred to as "isolated canonical singularities with a crepant blow up". We are interested in the case when the 4-cycle to be shrunk to a point is a special type of complex algebraic surface known as (generalised $\left.{ }^{3}\right)$ del Pezzo surface ${ }^{4} \mathrm{dP}_{n}[55,56]$. They are obtained by blowing up $\mathbb{P}^{2}$ at $0 \leq n \leq 8$ points. The non-vanishing Hodge numbers of these surfaces are

$$
h^{(0,0)}=h^{(2,2)}=1, \quad h^{(1,1)}=n+1 .
$$

The generators of $H^{(1,1)}\left(\mathrm{dP}_{n}\right)$ are given by a hyperplane class $H$ from $\mathbb{P}^{2}$ and exceptional divisors $E_{i}(i=1, \ldots, n)$ of the individual blow ups of $n$ points.

\footnotetext{
${ }^{3}$ Generalised refers to the fact that, in principle, these surfaces can have singularities themselves, see, e.g., [54] for definitions.

${ }^{4}$ More generally, Del Pezzo surfaces are complex 2-dimensional Fano surfaces, i.e., projective algebraic surfaces with ample anti-canonical divisor class $-K$ so that $-K \cdot C>0$ for each curve $C$. Among the Fano surfaces we find also $\mathbb{P}^{1} \times \mathbb{P}^{1}$ (sometimes also known as the Hirzebruch surface $\mathbb{F}_{0}$ ), with $h^{(1,1)}=2$.
} 


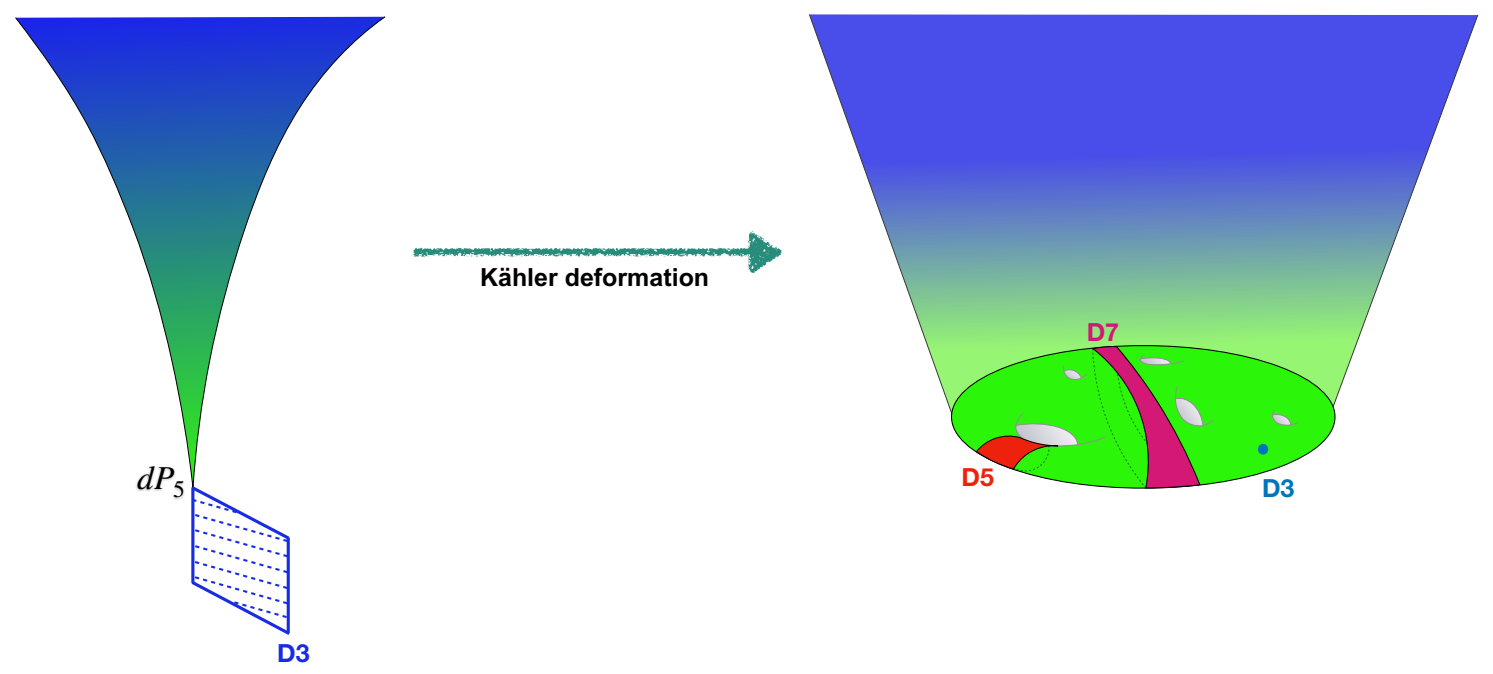

Figure 2. The splitting of a D3-brane at a del Pezzo singularity into fractional branes by considering the large volume limit of the corresponding 4-cycle.

\subsection{Large volume perspective of D3-branes at singularities}

In the following, we will denote the del Pezzo surface as $Y$, and consider the case in which it is embedded within a compact CY threefold $X$. When $Y$ collapses to zero size, this leads to a singular point in $X$. We now want to understand what happens when D-branes in IIB string theory probe this singular point.

In fact, we can obtain quite a bit of information by going to the B-model: the result of computing quantities such as the chiral spectrum or the superpotential in the B-model agree with the results in the full string theory [57, 58] (see [59] for a review). The computations in the B-model are insensitive to Kähler moduli deformations, so we can compute these protected quantities using classical geometry: we can resolve the singularity by deforming the Kähler structure to large cycle volumes and thereby small curvature. This gives rise to the large volume perspective [59-67] of a the D3-brane at the singularity as depicted in figure 2.

In doing this, it is important that the D3-brane can be understood as a bound state of so-called fractional branes $F_{i}$ supported on blow-up cycles (whether the D3 or the fractional branes are realised in any specific physical configuration depends on the Kähler data). These fractional branes are described by complexes of sheaves, which are intuitively vector bundles supported only on submanifolds. ${ }^{5}$ For instance, a D3-brane on a smooth point $p$ corresponds to a sky-scraper sheaf $\mathcal{O}_{p}$ : heuristically a "vector bundle" which is non-trivial at $p$ and trivial everywhere else.

It is convenient to represent the world-volume gauge theory as a 2-dimensional graph known as a quiver diagram. The total gauge group receives an additional factor $\mathrm{U}\left(N_{i}\right)$ for each stack of $N_{i}$ fractional brane $F_{i}$. In the quiver, we then draw a node with an assigned

\footnotetext{
${ }^{5}$ More precisely, the B-model branes should be understood as elements of the derived category of coherent sheaves of $X$. We refer the reader to [59] for an introduction to this formalism.
} 
gauge multiplet of $\mathrm{U}\left(N_{i}\right)$. Open strings stretched between two fractional branes $F_{i}$ and $F_{j}$ give rise to a massless spectrum of chiral multiplets in bi-fundamental representations of $\mathrm{U}\left(N_{i}\right) \times \mathrm{U}\left(N_{j}\right)$ which join pairs of nodes via directed lines. In the large volume description, these modes arise from elements of the groups $\operatorname{Ext}^{k}\left(F_{i}, F_{j}\right)$, which if $F_{i}$ and $F_{j}$ are ordinary vector bundles reduces to Dolbeault cohomology of the tensor bundle. See [59] for details of the general case.

We need to describe how to choose an appropriate basis of fractional branes. ${ }^{6}$ We will do so by focusing on exceptional collections, constructed as follows. A sheaf $F$ is called exceptional if $\operatorname{dim}(\operatorname{Hom}(F, F))=1$ as well as $\operatorname{Ext}^{k}(F, F)=0$ for all $k>0$. Similarly, a collection $\left\{F_{i}\right\}$ of sheaves is called exceptional if all $F_{i}$ are exceptional and, in addition, there exists an ordering such that $\operatorname{Ext}^{k}\left(F_{i}, F_{j}\right)=0$ for $i>j$ for any $k$, while $\operatorname{Ext}^{k}\left(F_{i}, F_{j}\right) \neq 0$ for one $k$ if $j>i$. There are systematic techniques to construct exceptional collections in the case of del Pezzo singularities (and more generally), we refer the reader to [63, 67-69] for the details.

The sheaves within an exceptional collection have the right property to describe the fractional branes having support on the internal cycles in the large volume perspective [63, 69, 70]. The property $\operatorname{Ext}^{k}\left(F_{i}, F_{i}\right)=0$ for all $k>0$ ensures that besides the gauge multiplet there is no adjoint matter in the world-volume gauge theory, i.e., there are no arrows beginning and ending at the same node. Moreover, the matter fields between two nodes have only one chirality due to the imposed ordering, that is, there is only a single direction for each arrow between any pairs of nodes.

In terms of string theory, any given fractional brane $F_{i}$ itself corresponds to a bound state of D7-, D5- and D3-brane states, cf. figure 2. In general some of these branes will be anti-branes, and at large volume the configuration badly breaks supersymmetry, but the fractional branes become mutually supersymmetric at the singular point (for appropriate choices of $B$-fields) due to $\alpha^{\prime}$ corrections $[58,59]$. Its RR-charges are combined into a charge vector $[71,72]$

$$
\operatorname{ch}\left(F_{i}\right)=\left(\operatorname{rk}\left(F_{i}\right), c_{1}\left(F_{i}\right), \operatorname{ch}_{2}\left(F_{i}\right)\right),
$$

corresponding to the D7-, D5- and D3-charge of $F_{i}$ respectively. The individual components are interpreted as follows:

- the D7-charge corresponding to the wrapping number of a D7-brane around the del Pezzo surface $Y$ is just given by the rank of $F_{i}$;

- the D5-charge is specified by the first Chern class $c_{1}\left(F_{i}\right)$ of $F_{i}$. That is, the D5 wrapping number $p_{i}^{A}$ around an integral basis of 2-cycles $\alpha_{A} \in H_{2}(Y)$ is given by

$$
p_{i}^{A}=\int_{\alpha_{A}} c_{1}\left(F_{i}\right)
$$

- the D3-brane charge represents a non-trivial instanton number which is obtained from the 2nd Chern character $\operatorname{ch}_{2}\left(F_{i}\right)$.

\footnotetext{
${ }^{6}$ The choice is not unique [68]. Different choices leading to the same IR physics are related Seiberg dualities [63, 69].
} 
Matter spectra and exceptional collections. As mentioned above, the massless matter spectrum of the gauge theory is encoded in $\operatorname{Ext}^{k}\left(F_{i}, F_{j}\right)$. For our purposes, it is sufficient to know the number of arrows between any two nodes. It is obtained from the relative Euler character

$$
\chi\left(F_{i}, F_{j}\right)=\sum_{k}(-1)^{k} \operatorname{dim}\left(\operatorname{Ext}^{k}\left(F_{i}, F_{j}\right)\right),
$$

which, after using the Riemann-Roch formula [71], becomes

$$
\chi\left(F_{i}, F_{j}\right)=\int_{Y} \operatorname{ch}\left(F_{i}^{*}\right) \operatorname{ch}\left(F_{j}\right) \operatorname{Td}(Y) .
$$

Here, $\operatorname{ch}\left(F_{i}\right)=\operatorname{rk}\left(F_{i}\right)+c_{1}\left(F_{i}\right)+\operatorname{ch}_{2}\left(F_{i}\right)$ and $\operatorname{Td}(Y)=1-\frac{1}{2} K+H^{2}$ are the Chern character of $F_{i}$ and the Todd class of $Y$ respectively $(-K$ is the anti-canonical divisor class). The equation (2.5) can then be expressed as

$$
\begin{aligned}
\chi\left(F_{i}, F_{j}\right)= & \operatorname{rk}\left(F_{i}\right) \operatorname{rk}\left(F_{j}\right)+\operatorname{rk}\left(F_{i}\right) \operatorname{ch}_{2}\left(F_{j}\right)+\operatorname{ch}_{2}\left(F_{i}\right) \operatorname{rk}\left(F_{j}\right) \\
& -c_{1}\left(F_{i}\right) \cdot c_{1}\left(F_{j}\right)+\frac{1}{2}\left(\operatorname{rk}\left(F_{j}\right) \operatorname{deg}\left(F_{i}\right)-\operatorname{rk}\left(F_{i}\right) \operatorname{deg}\left(F_{j}\right)\right)
\end{aligned}
$$

with $\operatorname{deg}\left(F_{i}\right)$ being the degree of $F_{i}$ defined as

$$
\operatorname{deg}\left(F_{i}\right)=-c_{1}\left(F_{i}\right) \cdot K
$$

in terms of the canonical class $K$ of $Y$. Note that by $(2.3) \operatorname{deg}\left(F_{i}\right)$ corresponds to the intersection number between the del Pezzo surface $Y$ and the D5-component of $F_{i}$. Furthermore, $\chi\left(F_{i}, F_{i}\right)=1$ corresponds to the presence of the gauge multiplet of the corresponding node. If this is true for all $F_{i}$ such as in exceptional collections, then this implies the absence of any adjoint matter.

One can show that $[63]$

$$
\sum_{j} \chi\left(F_{j}, F_{i}\right) N_{j}=0
$$

For the gauge theory, this implies that the total number of ingoing and outgoing lines are equal and hence the quiver gauge theory is free of non-abelian gauge anomalies. There can still be gauge anomalies associated to the U(1)-factors since there is mixing with closed string modes [73]. For an exceptional collection, (2.6) results in an upper triangular matrix with only 1's on the diagonal. The spectrum of chiral fields is completely contained in the anti-symmetrised expression of (2.6):

$$
\chi_{-}\left(F_{i}, F_{j}\right)=\chi\left(F_{i}, F_{j}\right)-\chi\left(F_{j}, F_{i}\right)=\operatorname{rk}\left(F_{j}\right) \operatorname{deg}\left(F_{i}\right)-\operatorname{rk}\left(F_{i}\right) \operatorname{deg}\left(F_{j}\right) .
$$

The direction of arrows is determined by the sign of $\chi_{-}\left(F_{i}, F_{j}\right)$. We can rewrite (2.8) for an exceptional collection as [71]

$$
\sum_{i} N_{i} \chi_{-}\left(F_{i}, F_{j}\right)=0
$$

This is again equivalent to the absence of non-abelian gauge anomalies. 


\subsection{Why branes at singularities?}

Del Pezzo surfaces are ubiquitous in CY manifolds. Their purpose for string model building is twofold. On the one hand, the possible rigidity of these 4-cycles makes them prime candidates to support non-perturbative effects contributing to the $4 \mathrm{~d}$ superpotential. On the other hand, the worldvolume theories of D3-branes at del Pezzo singularities obtained in the limit of vanishing 4-cycle volumes host interesting particle phenomenology. In particular:

1. Chiral matter fields in the worldvolume gauge theory of branes at singularities arise from intersections of 2- and 4-cycles. Every CY threefold singularity will have some 2- or 4-cycle volumes shrinking to zero size. The presence of chiral states necessitates the existence of 4-cycles collapsing to zero size. By our previous reasoning, $\mathrm{dP}_{n^{-}}$ singularities are the simplest examples associated with the vanishing volume limit of a single 4-cycle.

2. D-brane constructions of the SM from unoriented quivers come with one local anomalous $\mathrm{U}(1)$ corresponding to baryon number $\mathrm{U}(1)_{B}$. In the oriented covering quiver, we therefore must find two anomalous U(1)'s. Oriented del Pezzo quivers naturally come with precisely two anomalous U(1)'s. Geometrically speaking, this is due to two compact cycles in the non-compact CY geometry of a complex cone over del Pezzos. They can be identified with the canonical class and the del Pezzo surface itself $[72,74] .{ }^{7}$ From a field theory perspective, we would expect that for the $n+3$ fractional branes of a $\mathrm{dP}_{n}$-singularity, there exist $n+3$ gauge couplings and $n+3$ Fayet-Iliopoulos (FI) parameters. However, two of the latter are not freely tunable and associated with the two anomalous U(1)'s [76]. Although this is not specific to del Pezzo singularities per se, it is a favourable criterion for the models in our construction.

3. Fractional branes at the singularity are sufficient to generate a large variety of gauge groups together with the required matter spectrum. In particular, there is no necessity for the existence of flavour D7-branes, cf. Section 5 in [18]. Furthermore, turning on VEVs for bi-fundamental fields not only relates various $\mathrm{dP}_{n}$ models [63], but also has the potential of generating additional matter fields $[23,71]$.

Early local constructions based on oriented quivers at $\mathrm{dP}_{8}$-singularities [71] generated a gauge group containing the SM group. ${ }^{8}$ For toric $\mathrm{dP}_{n}$-singularities (i.e. $n \leq 3$ ), the authors of [77] showed that there are at most three families. ${ }^{9}$ Further, they argued that hierarchies

\footnotetext{
${ }^{7}$ In fact, a combination of U(1)'s is non-anomalous precisely when the associated collection of fractional brane has zero D7-charge (i.e. vanishing rank) as well as no intersection of the D5-component with the canonical class of the del Pezzo [75]. According to (2.9), this ensures the absence of chiral matter at the intersection of fractional branes and thus the absence of mixed anomalies. Branes wrapping the two compact Poincaré dual cycles, however, have chiral states inducing mixed anomalies in the U(1) worldvolume gauge theory.

${ }^{8}$ However, an embedding into a CY threefold retaining only the SM subgroup would require $h^{(1,1)} \geq 9$. This is because at least five 2-cycles of the local $\mathrm{dP}_{8}$ need to be non-trivial in the full $\mathrm{CY}$ to guarantee that the associated U(1) gauge fields become massive, see Section 5 in [18] for a discussion.

${ }^{9}$ We refer to [1] for a related result for orbifold singularities.
} 
in the quark masses can indeed be realised for $n \geq 1$. As discussed in [78], D3-branes at $\mathrm{dP}_{n}$-singularities with $n>2$ are favourable to realise the hierarchical mixing angles in the CKM matrix. A brief discussion of higher $\mathrm{dP}_{n}$-singularities together with constraints for global embeddings can be found in [18]. Further works found realistic extensions of the $\mathrm{SM}$ in global orientifolded models [19-21] together with inflation [22].

\subsection{The $\mathrm{dP}_{5}$-quiver and its involutions}

In the rest of this section, we will use the ideas reviewed above to construct the (MS)SM as a local model on a system of fractional branes at a $\mathrm{dP}_{n}$-singularity. Let us start by identifying the minimal value of $n$ to realise the Minimal Quiver Standard Model (MQSM) [25, 26]. The MQSM requires at least 3 nodes for both oriented and unoriented quivers [79]. Nonsupersymmetric unoriented versions of the MQSM were proposed in [25, 26] with the minimally required number of 3 nodes. The seminal work [23] found analogous supersymmetric constructions of the MQSM from D3-branes at $\mathrm{dP}_{5}$-singularities. In fact, the $\mathrm{dP}_{5}$ quiver is the minimal del Pezzo quiver to contain the MQSM. For this reason, we are particularly interested in the two unoriented models based on $\mathrm{dP}_{5}$ implemented in [23].

We begin by analysing the quiver gauge theory for a D3-brane at a $\mathrm{dP}_{5}$-singularity [23, 63]. The singularity itself has a well-known toric limit corresponding to the $\mathbb{Z}_{2} \times \mathbb{Z}_{2}$ orbifold of the conifold. $\mathbb{P}^{2}$ itself has three independent holomorphic cycles, i.e., the class of points, the class of the hyperplane and the class of the 4-cycle. With the additional 5 blown up points, we expect to find 8 nodes in the $\mathrm{dP}_{5}$ quiver diagram. It is easily constructed from intersection numbers of fractional branes.

For del Pezzo surfaces, we choose a basis of generators of $H_{2}\left(\mathrm{dP}_{n}, \mathbb{Z}\right)$ such that

$$
H \cdot H=1, \quad E_{i} \cdot E_{j}=-\delta_{i j}, \quad H \cdot E_{i}=0 .
$$

Here, $H$ is the hyperplane class, while the $E_{i}$ are $n$ exceptional curves from blowing up points in $\mathbb{P}^{2}$. The canonical class of a del Pezzo surface is given by

$$
K\left(\mathrm{dP}_{n}\right)=-3 H+\sum_{i=1}^{n} E_{i} .
$$

The exceptional collection in [23] is characterised by the charge vectors

$$
\begin{array}{ll}
\operatorname{ch}\left(F_{1}\right)=\left(1, H-E_{1}, 0\right) & \operatorname{deg}\left(F_{1}\right)=2, \\
\operatorname{ch}\left(F_{2}\right)=\left(1, H-E_{2}, 0\right) & \operatorname{deg}\left(F_{2}\right)=2, \\
\operatorname{ch}\left(F_{3}\right)=-\left(1,2 H-E_{1}-E_{2}-E_{4}, \frac{1}{2}\right) & \operatorname{deg}\left(F_{3}\right)=-3, \\
\operatorname{ch}\left(F_{4}\right)=-\left(1,2 H-E_{1}-E_{2}-E_{5}, \frac{1}{2}\right) & \operatorname{deg}\left(F_{4}\right)=-3, \\
\operatorname{ch}\left(F_{5}\right)=-\left(1, H-E_{3}, 0\right), & \operatorname{deg}\left(F_{5}\right)=-2, \\
\operatorname{ch}\left(F_{6}\right)=-\left(1, E_{4}+E_{5},-1\right) & \operatorname{deg}\left(F_{6}\right)=-2, \\
\operatorname{ch}\left(F_{7}\right)=\left(1, H, \frac{1}{2}\right) & \operatorname{deg}\left(F_{7}\right)=3, \\
\operatorname{ch}\left(F_{8}\right)=\left(1,2 H-E_{1}-E_{2}-E_{3}, \frac{1}{2}\right) & \operatorname{deg}\left(F_{8}\right)=3 .
\end{array}
$$




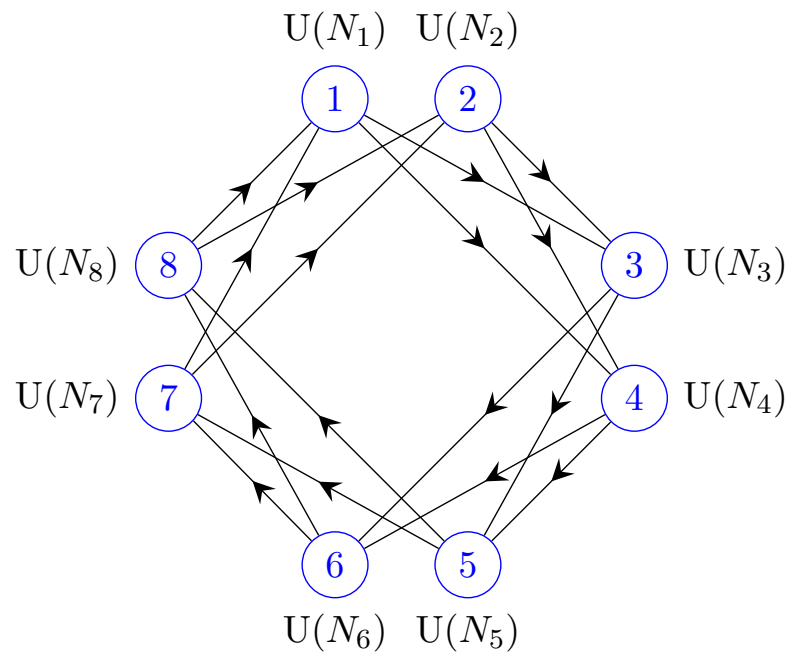

Figure 3. Quiver diagram for a D3-brane at a $\mathrm{dP}_{5}$-singularity.

Since this is an exceptional collection, we use (2.9) to compute the number of fields between each node. We find

$$
\chi_{-}\left(F_{i}, F_{j}\right)=\left(\begin{array}{cccccccc}
0 & 0 & 1 & 1 & 0 & 0 & -1 & -1 \\
0 & 0 & 1 & 1 & 0 & 0 & -1 & -1 \\
-1 & -1 & 0 & 0 & 1 & 1 & 0 & 0 \\
-1 & -1 & 0 & 0 & 1 & 1 & 0 & 0 \\
0 & 0 & -1 & -1 & 0 & 0 & 1 & 1 \\
0 & 0 & -1 & -1 & 0 & 0 & 1 & 1 \\
1 & 1 & 0 & 0 & -1 & -1 & 0 & 0 \\
1 & 1 & 0 & 0 & -1 & -1 & 0 & 0
\end{array}\right) .
$$

The corresponding quiver diagram is shown in figure 3. We observe that there is at most one line between each node. The direction of the arrow between node $i$ and $j$ is determined by the sign of $\chi_{-}\left(F_{i}, F_{j}\right)$.

Several key aspects of the worldvolume theory deserve further scrutiny, namely gauge anomaly cancellation, anomalous U(1)'s and orientifold involutions.

Gauge anomalies. Gauge anomalies in quiver gauge theories are easily computed through counting incoming and outgoing arrows at the various nodes, see e.g. section 2 in [80]. Let us denote with $I_{i j}$ an edge between nodes $i$ and $j$. Then we define

$$
\sigma\left(I_{i j}, i\right)= \begin{cases}+1 & i \text { endpoint } \\ -1 & i \text { startingpoint } \\ 0 & \nexists I_{i j}=\text { no edge } .\end{cases}
$$

For each node $i$, anomaly cancellation is ensured if

$$
\sum_{j} \sigma\left(I_{i j}, i\right) N_{j}=0
$$


This is equivalent to the statement in eq. (2.10). For the $\mathrm{dP}_{5}$-quiver in figure 3 , we obtain the two conditions

$$
\begin{aligned}
& N_{1}+N_{2}=N_{5}+N_{6}, \\
& N_{3}+N_{4}=N_{7}+N_{8} .
\end{aligned}
$$

The models to be discussed below fall into two classes satisfying the above constraints through the choices

- model I:

$$
N_{3}=N_{8}, \quad N_{4}=N_{7}, \quad N_{1}=N_{5}, \quad N_{2}=N_{6} ;
$$

- model II:

$$
N_{3}=N_{8}, \quad N_{4}=N_{7}, \quad N_{5}=N_{6}, \quad N_{1}+N_{2}=2 N_{5} .
$$

Below, we argue that the individual choices require distinct types of orientifold involutions.

U(1) charges. Each of the 8 nodes of the quiver in figure 3 comes with an $\mathrm{U}(1)$ factor. The U(1) charges $q_{i}$ of the corresponding fields can be determined by associating to each ingoing arrow +1 , to each outgoing arrow -1 and to no arrow 0 . Thus, we find for each field $X_{a b}$ the charge vectors $\mathbf{q}^{(a b)}$ with entries $q_{i}^{(a b)}, i=1, \ldots, 8$, and

$$
q_{i}^{(a b)}=\delta_{i}^{a}-\delta_{i}^{b}
$$

so that for instance

$$
\mathbf{q}^{(13)}=(1,0,-1,0,0,0,0,0)
$$

Out of these $8 \mathrm{U}(1)$ 's two have to be anomalous according to our previous reasoning.

In order to find the anomaly-free and anomalous U(1)'s we can look for combinations of the type

$$
Q_{i}=\sum_{j=1}^{8} C_{i j} \frac{q_{j}}{N_{j}} .
$$

Normalising $q_{i} \cdot q_{j}=N_{i} N_{j} \delta_{i j}$ we find an orthogonal basis for the 6 non-anomalous U(1)'s as follows:

$$
\begin{aligned}
Q_{1} & =\sum_{j=1}^{8} \frac{q_{j}}{N_{j}} & Q_{2} & =\frac{q_{1}}{N_{1}}-\frac{q_{2}}{N_{2}} \\
Q_{3} & =\frac{q_{3}}{N_{3}}-\frac{q_{4}}{N_{4}} & Q_{4} & =\frac{q_{5}}{N_{5}}-\frac{q_{6}}{N_{6}} \\
Q_{5} & =\frac{q_{7}}{N_{7}}-\frac{q_{8}}{N_{8}} & Q_{6} & =\frac{q_{1}}{N_{1}}+\frac{q_{2}}{N_{2}}-\frac{q_{3}}{N_{3}}-\frac{q_{4}}{N_{4}}+\frac{q_{5}}{N_{5}}+\frac{q_{6}}{N_{6}}-\frac{q_{7}}{N_{7}}-\frac{q_{8}}{N_{8}}
\end{aligned}
$$




\begin{tabular}{|c|c|c|c|c|c|c|c|c|}
\hline Field & $Q_{1}$ & $Q_{2}$ & $Q_{3}$ & $Q_{4}$ & $Q_{5}$ & $Q_{6}$ & $Q_{7}$ & $Q_{8}$ \\
\hline$X_{13}$ & $\frac{1}{N_{1}}-\frac{1}{N_{3}}$ & $\frac{1}{N_{1}}$ & $-\frac{1}{N_{3}}$ & 0 & 0 & $\frac{1}{N_{1}}+\frac{1}{N_{3}}$ & $\frac{1}{N_{1}}$ & $-\frac{1}{N_{3}}$ \\
\hline$X_{14}$ & $\frac{1}{N_{1}}-\frac{1}{N_{4}}$ & $\frac{1}{N_{1}}$ & $\frac{1}{N_{4}}$ & 0 & 0 & $\frac{1}{N_{1}}+\frac{1}{N_{4}}$ & $\frac{1}{N_{1}}$ & $-\frac{1}{N_{4}}$ \\
\hline$X_{23}$ & $\frac{1}{N_{2}}-\frac{1}{N_{3}}$ & $-\frac{1}{N_{2}}$ & $-\frac{1}{N_{3}}$ & 0 & 0 & $\frac{1}{N_{2}}+\frac{1}{N_{3}}$ & $\frac{1}{N_{2}}$ & $-\frac{1}{N_{3}}$ \\
\hline$X_{24}$ & $\frac{1}{N_{2}}-\frac{1}{N_{4}}$ & $-\frac{1}{N_{2}}$ & $\frac{1}{N_{4}}$ & 0 & 0 & $\frac{1}{N_{2}}+\frac{1}{N_{4}}$ & $\frac{1}{N_{2}}$ & $-\frac{1}{N_{4}}$ \\
\hline$X_{35}$ & $\frac{1}{N_{3}}-\frac{1}{N_{5}}$ & 0 & $\frac{1}{N_{3}}$ & $-\frac{1}{N_{5}}$ & 0 & $-\frac{1}{N_{3}}-\frac{1}{N_{5}}$ & $\frac{1}{N_{5}}$ & $\frac{1}{N_{3}}$ \\
\hline$X_{36}$ & $\frac{1}{N_{3}}-\frac{1}{N_{6}}$ & 0 & $\frac{1}{N_{3}}$ & $\frac{1}{N_{6}}$ & 0 & $-\frac{1}{N_{3}}-\frac{1}{N_{6}}$ & $\frac{1}{N_{6}}$ & $\frac{1}{N_{3}}$ \\
\hline$X_{45}$ & $\frac{1}{N_{4}}-\frac{1}{N_{5}}$ & 0 & $-\frac{1}{N_{4}}$ & $-\frac{1}{N_{5}}$ & 0 & $-\frac{1}{N_{4}}-\frac{1}{N_{5}}$ & $\frac{1}{N_{5}}$ & $\frac{1}{N_{4}}$ \\
\hline$X_{46}$ & $\frac{1}{N_{4}}-\frac{1}{N_{6}}$ & 0 & $-\frac{1}{N_{4}}$ & $\frac{1}{N_{6}}$ & 0 & $-\frac{1}{N_{4}}-\frac{1}{N_{6}}$ & $\frac{1}{N_{6}}$ & $\frac{1}{N_{4}}$ \\
\hline$X_{57}$ & $\frac{1}{N_{5}}-\frac{1}{N_{7}}$ & 0 & 0 & $\frac{1}{N_{5}}$ & $-\frac{1}{N_{7}}$ & $\frac{1}{N_{5}}+\frac{1}{N_{7}}$ & $-\frac{1}{N_{5}}$ & $\frac{1}{N_{7}}$ \\
\hline$X_{58}$ & $\frac{1}{N_{5}}-\frac{1}{N_{8}}$ & 0 & 0 & $\frac{1}{N_{5}}$ & $\frac{1}{N_{8}}$ & $\frac{1}{N_{5}}+\frac{1}{N_{8}}$ & $-\frac{1}{N_{5}}$ & $\frac{1}{N_{8}}$ \\
\hline$X_{67}$ & $\frac{1}{N_{6}}-\frac{1}{N_{7}}$ & 0 & 0 & $-\frac{1}{N_{6}}$ & $-\frac{1}{N_{7}}$ & $\frac{1}{N_{6}}+\frac{1}{N_{7}}$ & $-\frac{1}{N_{6}}$ & $\frac{1}{N_{7}}$ \\
\hline$X_{68}$ & $\frac{1}{N_{6}}-\frac{1}{N_{8}}$ & 0 & 0 & $-\frac{1}{N_{6}}$ & $\frac{1}{N_{8}}$ & $\frac{1}{N_{6}}+\frac{1}{N_{8}}$ & $-\frac{1}{N_{6}}$ & $\frac{1}{N_{8}}$ \\
\hline$X_{71}$ & $\frac{1}{N_{7}}-\frac{1}{N_{1}}$ & $-\frac{1}{N_{1}}$ & 0 & 0 & $\frac{1}{N_{7}}$ & $-\frac{1}{N_{7}}-\frac{1}{N_{1}}$ & $-\frac{1}{N_{1}}$ & $-\frac{1}{N_{7}}$ \\
\hline$X_{72}$ & $\frac{1}{N_{7}}-\frac{1}{N_{2}}$ & $\frac{1}{N_{2}}$ & 0 & 0 & $\frac{1}{N_{7}}$ & $-\frac{1}{N_{7}}-\frac{1}{N_{2}}$ & $-\frac{1}{N_{2}}$ & $-\frac{1}{N_{7}}$ \\
\hline$X_{81}$ & $\frac{1}{N_{8}}-\frac{1}{N_{1}}$ & $-\frac{1}{N_{1}}$ & 0 & 0 & $-\frac{1}{N_{8}}$ & $-\frac{1}{N_{8}}-\frac{1}{N_{1}}$ & $-\frac{1}{N_{1}}$ & $-\frac{1}{N_{8}}$ \\
\hline$X_{82}$ & $\frac{1}{N_{8}}-\frac{1}{N_{2}}$ & $\frac{1}{N_{2}}$ & 0 & 0 & $-\frac{1}{N_{8}}$ & $-\frac{1}{N_{8}}-\frac{1}{N_{2}}$ & $-\frac{1}{N_{2}}$ & $-\frac{1}{N_{8}}$ \\
\hline
\end{tabular}

Table 1. List of U(1) charges for the bi-fundamentals appearing in the quiver diagram 3.

The 2 anomalous ${ }^{10} \mathrm{U}(1)$ 's are:

$$
Q_{7}=\frac{q_{1}}{N_{1}}+\frac{q_{2}}{N_{2}}-\frac{q_{5}}{N_{5}}-\frac{q_{6}}{N_{6}}, \quad Q_{8}=\frac{q_{3}}{N_{3}}+\frac{q_{4}}{N_{4}}-\frac{q_{7}}{N_{7}}-\frac{q_{8}}{N_{8}} .
$$

One can check that these $\mathrm{U}(1)$ charges are othorgonal so that

$$
Q_{i} Q_{j}=\operatorname{diag}(8,2,2,2,2,8,4,4) .
$$

We summarised the $\mathrm{U}(1)$ charges of the bi-fundamentals $X_{i j}$ in table 1 .

Orientifold involutions - models I and II. In this section, we define two unoriented models based on different orientifold actions. In general, we require that the orientifold involution leaves the superpotential invariant. For simplicity, we restrict to USp-projections.

\footnotetext{
${ }^{10}$ It turns out that all rank zero and degree zero combinations of fractional branes are free of anomalies because the chiral spectrum from (2.9) vanishes [72]. Indeed, we find that only $Q_{7}$ and $Q_{8}$ have nonvanishing ranks and degrees corresponding to the expected two anomalous U(1)'s.
} 
Model I

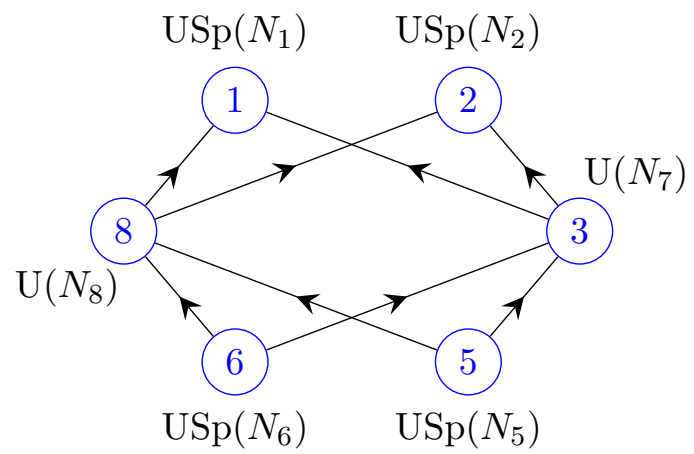

Model II

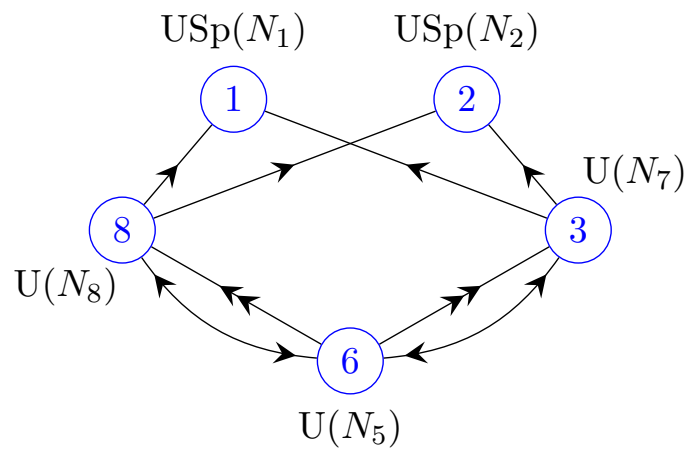

Figure 4. Unoriented quiver diagrams for model I (left) and model II (right).

In the toric limit, the superpotential is obtained from a dimer diagram and given by [23]

$$
\begin{aligned}
W_{Q}^{\text {toric }}= & X_{13} X_{35} X_{58} X_{81}-X_{14} X_{46} X_{68} X_{81}+X_{14} X_{45} X_{57} X_{71}-X_{13} X_{36} X_{67} X_{71} \\
& +X_{24} X_{46} X_{67} X_{72}-X_{23} X_{35} X_{57} X_{72}+X_{23} X_{36} X_{68} X_{82}-X_{24} X_{45} X_{58} X_{82}
\end{aligned}
$$

The first model referred to as model $I$ in the following in analogy to [23] corresponds to the orientifold action

$$
1 \leftrightarrow 1^{*}, \quad 2 \leftrightarrow 2^{*}, \quad 3 \leftrightarrow 8^{*}, \quad 4 \leftrightarrow 7^{*}, \quad 5 \leftrightarrow 5^{*}, \quad 6 \leftrightarrow 6^{*} .
$$

This is consistent with (2.19) and implies that

$$
\begin{array}{llll}
X_{81}=X_{13}^{T} \gamma_{1}, & X_{71}=a^{-1} X_{14}^{T} \gamma_{1}, & X_{82}=b^{-1} X_{23}^{T} \gamma_{2}, & X_{72}=X_{24}^{T} \gamma_{2}, \\
X_{68}=\gamma_{6}^{-1} X_{36}^{T}, & X_{58}=\gamma_{5}^{-1} X_{35}^{T}, & X_{67}=\gamma_{6}^{-1} X_{46}^{T}, & X_{57}=\gamma_{5}^{-1} X_{45}^{T},
\end{array}
$$

in terms of some phases $a$ and $b$ and $\gamma_{i}^{T}=-\gamma_{i}$ for USp-projections. The resulting quiver diagram is depicted on the left side of figure 4. For the toric superpotential (2.27), the projection on node 1 and 5 as well as 2 and 6 must always be identical.

There is a second class of models called model II in [23] satisfying (2.19). The orientifold action reads

$$
1 \leftrightarrow 1^{*}, \quad 2 \leftrightarrow 2^{*}, \quad 3 \leftrightarrow 8^{*}, \quad 4 \leftrightarrow 7^{*}, \quad 5 \leftrightarrow 6^{*} .
$$

This implies that the fields are fixed as

$$
\begin{array}{llll}
X_{81}=X_{13}^{T} \gamma_{1}, & X_{71}=X_{14}^{T} \gamma_{2}, & X_{82}=X_{23}^{T} \gamma_{2}, & X_{72}=X_{24}^{T} \gamma_{2}, \\
X_{68}=X_{35}^{T}, & X_{58}=X_{36}^{T}, & X_{67}=X_{45}^{T}, & X_{57}=X_{46}^{T} .
\end{array}
$$

The quiver diagram is shown on the right of figure 4 .

An important question concerns gauge anomalies in the orientifolded quivers. This becomes an issue whenever the projection acts differently on the positive and negative 
contributions to an anomaly $[23,80]$. It is typically the case for orientifolded gauge theories containing (anti-)symmetric tensor representations which arise for fixed points on edges. These representations contribute $N \pm 4$ to the anomaly coefficient rather than $N$ as for the bi-fundamentals in the parent theory, see e.g. footnote 2 of [79]. In both of our models, there are no fixed points on edges and consequently orientifolding does not change the anomaly cancellation conditions which is consistent with the findings of [81].

\subsection{Higgsing the $\mathrm{dP}_{5}$ quiver gauge theory - model $\mathrm{I}$}

The two unoriented quivers in figure 4 contain already the chiral spectrum of the MSSM, albeit only one family of each quarks and leptons. The necessary number of chiral families together with the right amount of non-chiral matter are obtained from a higher rank gauge theory by turning on suitable Vacuum Expectation Values (VEVs) for bi-fundamental matter fields. This section is concerned with this Higgsing procedure for model I of the $\mathrm{dP}_{5}$ quiver. A similar analysis for model II is summarised in appendix C.3. The Higgsing works essentially in a two step procedure by first obtaining a version of a left-right symmetric model $^{11} \mathrm{U}(3) \times \mathrm{USp}(2)_{L} \times \mathrm{USp}(2)_{R} \times \mathrm{U}(1)$ which subsequently needs to be reduced to the (MS)SM gauge group via conventional Higgsing $\mathrm{USp}(2)_{R} \times \mathrm{U}(1) \rightarrow \mathrm{U}(1)[23,79]$.

Breaking patterns for bi-fundamental VEVs. We begin our analysis with outlining the breaking pattern for fields in bi-fundamental representations of $\mathrm{U}\left(N_{1}\right) \times \mathrm{U}\left(N_{2}\right)$. We choose a basis of generators $T_{j} \in\left\{h_{0}, h_{i}, E_{p q}^{+}, E_{p q}^{-}\right\}, j=1, \ldots, N^{2}$, for the Lie algebra $\mathfrak{u}(N)$ by defining

$$
\begin{array}{rlrlrl}
h_{i} & =\mathrm{i}\left(e_{i, i}-e_{i+1, i+1}\right), & i & =1,2, \ldots, N-1 & & \\
E_{p q}^{-} & =e_{p q}-e_{q p}, & & p, q=1,2, \ldots, N, & & p<q \\
E_{p q}^{+} & =\mathrm{i}\left(e_{p q}+e_{q p}\right), & & p, q=1,2, \ldots, N, & & p<q \\
h_{0} & =\mathrm{i} \mathbb{1}_{N} & &
\end{array}
$$

where $e_{p q}$ are the $N \times N$-matrices $\left(e_{p q}\right)_{i k}=\delta_{i p} \delta_{k q}$. In the remainder of this section, we look at the gauge-invariant kinetic terms for different bi-fundamental fields $\left(\mathbf{N}_{1}, \overline{\mathbf{N}}_{2}\right)$ analysing the breaking patterns for distinct choices of VEVs through the resulting mass matrices for gauge bosons.

As a warm-up, we consider a bi-fundamental field $X$ in $(\mathbf{N}, \overline{\mathbf{N}})$ of $\mathrm{U}(N)^{2}$. This is a simple setup for a matter field between two nodes of $N$ fractional branes. We may choose VEVs of the form

$$
\langle X\rangle=\operatorname{diag}\left(a_{1}, \ldots, a_{N}\right) .
$$

This choice is in unitary gauge where all Goldstone modes are being absorbed by massive gauge bosons to become the longitudinal degree of freedom. The group $\mathrm{U}(N)^{2}$ is broken to a subgroup $H$ depending on the choice of constants $a_{i}$. Here, we distinguish three scenarios. First, if all the $a_{i}$ are inequivalent, then $H=\mathrm{U}(1)^{N}$ so that the number of Goldstone modes is $2 N^{2}-N$. The original field $X$ encodes $2 N^{2}$ real degrees of freedom

\footnotetext{
${ }^{11}$ Recall that there exists an accidental isomorphism $\mathrm{USp}(2) \cong \mathrm{SU}(2)$ with both being used interchangeably in what follows.
} 


\begin{tabular}{|cc|c|c|}
\hline$\langle X\rangle$ & groups & choices \\
\hline \hline$\left(\begin{array}{ccc}a_{1} \mathbb{1}_{N_{1} \times N_{1}} & 0_{N_{1} \times N_{2}} & 0_{N_{1} \times M} \\
0_{N_{2} \times N_{1}} & a_{2} \mathbb{1}_{N_{2} \times N_{2}} & 0_{N_{2} \times M} \\
0_{N_{2} \times N_{1}} & a_{3} \mathbb{1}_{N_{2} \times N_{2}} & 0_{N_{2} \times M}\end{array}\right)$ & $G=\mathrm{U}\left(N_{1}+2 N_{2}\right) \times \mathrm{U}\left(N_{1}+N_{2}+M\right)$ & $a_{2}, a_{3} \neq 0$ \\
$a_{2}=0$ \\
$a_{3}=0$ \\
\hline$\left(\begin{array}{ccc}a_{1} \mathbb{1}_{N_{1} \times N_{1}} & a_{2} \mathbb{1}_{N_{1} \times N_{1}} & 0_{N_{1} \times M} \\
0_{N_{2} \times N_{1}} & 0_{N_{2} \times N_{1}} & 0_{N_{2} \times M}\end{array}\right)$ & $G=\mathrm{U}\left(N_{1}\right) \times \mathrm{U}\left(N_{2}\right)^{2} \times \mathrm{U}(M)$ & $a_{2}=0$ \\
\hline$\left(\begin{array}{ccc}a_{1} \mathbb{1}_{N_{1} \times N_{1}} & 0_{N_{1} \times N_{2}} & 0_{N_{1} \times M} \\
a_{2} \mathbb{1}_{N_{1} \times N_{1}} & 0_{N_{1} \times N_{2}} & 0_{N_{1} \times M} \\
0_{N_{2} \times N_{1}} & a_{3} \mathbb{1}_{N_{2} \times N_{2}} & 0_{N_{2} \times M}\end{array}\right)$ & $G=\mathrm{U}\left(2 N_{1}+N_{2}\right) \times \mathrm{U}\left(2 N_{1}+M\right)$ & $a_{2} \neq 0$ \\
\hline
\end{tabular}

Table 2. Breaking patterns of bi-fundamental matter fields $X$ for convenient block-like structures.

so that after Higgsing $N$ potentially massive fields (the Higgs bosons) remain. The second scenario considers $a_{i}=a$ for all $i$ which implies $H=\mathrm{U}(N)$. The number of Goldstone modes is $2 N^{2}-N^{2}=N^{2}$ so that $N^{2}$ real degrees of freedom remain as Higgs fields in the adjoint of $\mathrm{U}(N)$. Finally, the third configuration consists of block-like structures

$$
\langle X\rangle=\operatorname{diag}(\underbrace{a_{1}, \ldots, a_{1}}_{n_{1}}, \underbrace{a_{2}, \ldots, a_{2}}_{n_{2}}, \ldots, \underbrace{a_{M}, \ldots, a_{M}}_{n_{M}}), \quad \sum_{k=1}^{M} n_{k}=N
$$

so that $H=\mathrm{U}\left(n_{1}\right) \times \ldots \times \mathrm{U}\left(n_{M}\right)$. In this case, we find $2 N^{2}-\sum_{i=1}^{M} n_{i}^{2}$ Goldstone modes and $n_{i}^{2}$ Higgses survive in the adjoint of $\mathrm{U}\left(n_{i}\right)$ for all $i=1, \ldots, M$.

The next stage is a matter field $X$ in the bi-fundamental $(\mathbf{N}+\mathbf{M}, \overline{\mathbf{N}})$ of $\mathrm{U}(N+M) \times$ $\mathrm{U}(N)$. A VEV of the form

$$
\langle X\rangle=\left(\begin{array}{c}
\operatorname{diag}\left(a_{1}, \ldots, a_{N}\right) \\
0_{M \times N}
\end{array}\right)
$$

breaks the group to $H=\mathrm{U}(M) \times \mathrm{U}(N)$ for $a_{i}=a$ for all $i$. The number of Goldstone modes is given by $(N+M)^{2}+N^{2}-M^{2}-N^{2}=2 M N+N^{2}$, while the number of surviving matter fields is $2(N+M) N-\left(2 M N+N^{2}\right)=N^{2}$ in the adjoint of $\mathrm{U}(N)$.

More generally, we also make heavy use of the breaking patterns summarised in table 2 . We indicate various possible choices of constants in the third column that do not change the breaking pattern, but modify superpotential couplings. Below, we require several different versions of these VEVs to break the original $\mathrm{dP}_{5}$ quiver gauge theory in figure 3 to a covering quiver of the (MS)SM.

Higgsing - building bound states of fractional branes. The quiver gauge theory obtained at the $\mathrm{dP}_{5}$ singularity contains 8 factors of unitary groups together with 16 bifundamentals. The breaking pattern is thus significantly more involved. To gain some intuition, we begin by outlining the process of condensing several nodes in the original 
quiver to a single node. We follow the ideas of [23] by building bound states of fractional branes. In the Higgsed quiver, the new node is associated with $n$ copies of a new (bound state) fractional brane with associated gauge group $\mathrm{U}(n)$.

In the remainder of this section, we focus on model I. Here, the basic working plan is to collapse nodes $1,2,5,6$ in figure 3 (together with a sub-sector at nodes $3,4,7,8$ ) to two individual U(2)'s. Having achieved this, we only need to ensure that the unbroken symmetry groups at nodes $3,4,7,8$ are $\mathrm{U}(3)$ and $\mathrm{U}(1)$. Thus, after orientifolding, we end up with the left-right symmetric model.

All in all, these arguments necessitate the construction of two bound states associated with the two U(2)'s. Practically, bound states are built by combining the charge vectors of fractional branes in the exceptional collection $\left\{F_{1}, \ldots, F_{8}\right\}$. To find suitable linear combinations, we recall that the intersection numbers $\chi_{-}\left(F_{i}, F_{j}\right)$ of the new basis of 6 fractional branes count additional chiral families. Looking at (2.9), we obtain exactly three chiral families if we replace four of our original fractional branes $\left\{F_{1}, F_{2}, F_{5}, F_{6}\right\}$ by two rank \pm 3 and degree $\mp 6$ objects $\left\{F_{a}, F_{b}\right\}$. More explicitly, this implies that for instance

$$
\chi_{-}\left(F_{1}, F_{3}\right)=1 \quad \rightarrow \quad \chi_{-}\left(F_{a}, F_{3}\right)=3, \quad \chi_{-}\left(F_{b}, F_{3}\right)=-3,
$$

and similarly for all other nodes. We stress that the chiral intersection matrix (2.9) only depends on the rank and degree of fractional branes. At this level of discussion, the nonchiral spectrum remains undetermined and must be analysed separately below.

The first linear combination to satisfy our constraints is defined as

$$
\operatorname{ch}\left(F_{b}\right)=\sum_{i=1}^{8} n_{i}^{(b)} \operatorname{ch}\left(F_{i}\right)=-\left(3,2 H-E_{2}-E_{3}+E_{4}+E_{5},-2\right), \quad \operatorname{deg}\left(F_{b}\right)=-6
$$

in terms of the 8-vector

$$
\mathbf{n}^{(b)}=(1,0,1,1,2,2,1,1) .
$$

The bound state $2 F_{b}$ is realised by turning on VEVs

$$
\begin{array}{lll}
\left\langle X_{13}\right\rangle=\left(a_{1}\right), & \left\langle X_{14}\right\rangle=\left(b_{1}\right), & \left\langle X_{36}\right\rangle=\left(\begin{array}{ll}
y_{1} & 0
\end{array}\right), \\
\left\langle X_{46}\right\rangle=\left(\begin{array}{ll}
0 w_{2}
\end{array}\right), & \left\langle X_{35}\right\rangle=\left(\begin{array}{ll}
x_{1} 0
\end{array}\right), & \left\langle X_{45}\right\rangle=\left(\begin{array}{ll}
0 & z_{2}
\end{array}\right)
\end{array}
$$

where each entry corresponds to a $2 \times 2$-matrix. We also fix the $\gamma$-matrices

$$
\gamma_{1}=\mathrm{i} \sigma_{2}=\left(\begin{array}{cc}
0 & 1 \\
-1 & 0
\end{array}\right), \quad \gamma_{5}=\gamma_{6}=\left(\begin{array}{cc}
\mathrm{i} \sigma_{2} & 0 \\
0 & \mathrm{i} \sigma_{2}
\end{array}\right)
$$

so that under orientifold projection the remaining VEVs are fixed through (2.29) with $a=1$. Notice that $\gamma_{i}^{T}=-\gamma_{i}$ as required for a $U S p$-projection. We consider the partial quiver diagram for this bound state on the left hand side in figure 5 and find that indeed the above VEVs imply the breaking pattern

$$
\mathrm{U}(2)^{5} \times \mathrm{U}(4)^{2} \rightarrow \mathrm{U}(2) \stackrel{\text { Orientifolding }}{\longrightarrow} \mathrm{USp}(2) .
$$


Bound state $2 F_{b}$

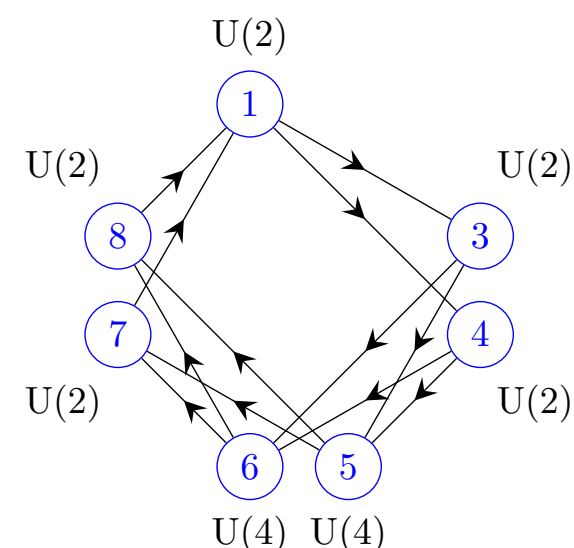

Bound state $2 F_{a}$

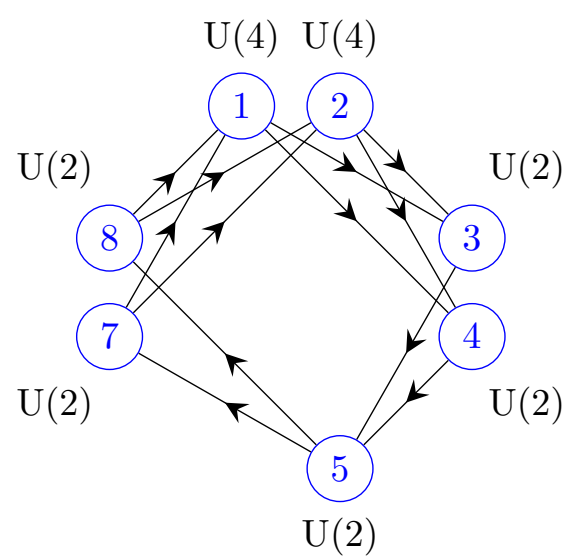

Figure 5. Partial quiver for the bound states $2 F_{b}$ (left) and $2 F_{a}$ (right) of model I.

In the oriented model, we have 48 Goldstone modes which leaves us with 64 real scalars in adjoints of $\mathrm{U}(2)$.

The second bound state can be written as

$$
\operatorname{ch}\left(F_{a}\right)=\sum_{i=1}^{8} n_{i}^{(a)} \operatorname{ch}\left(F_{i}\right)=\left(3,2 H-E_{1}-E_{2}+E_{4}+E_{5}, 0\right), \quad \operatorname{deg}\left(F_{a}\right)=6
$$

where

$$
\mathbf{n}^{(a)}=(2,2,1,1,1,0,1,1) .
$$

We claim that the bound state $2 F_{a}$ can be achieved by turning on VEVs

$$
\begin{aligned}
& \left\langle X_{13}\right\rangle=\left(\begin{array}{c}
a_{2} \\
0
\end{array}\right), \quad\left\langle X_{14}\right\rangle=\left(\begin{array}{c}
0 \\
b_{3}
\end{array}\right), \quad\left\langle X_{23}\right\rangle=\left(\begin{array}{c}
c_{1} \\
0
\end{array}\right), \\
& \left\langle X_{24}\right\rangle=\left(\begin{array}{c}
0 \\
d_{2}
\end{array}\right), \quad\left\langle X_{35}\right\rangle=\left(x_{3}\right), \quad\left\langle X_{45}\right\rangle=\left(z_{3}\right)
\end{aligned}
$$

where each entry corresponds to a $2 \times 2$-matrix. We also fix the $\gamma$-matrices in (2.29) through

$$
\gamma_{5}=\mathrm{i} \sigma_{2}=\left(\begin{array}{cc}
0 & 1 \\
-1 & 0
\end{array}\right), \quad \gamma_{1}=\gamma_{2}=\left(\begin{array}{cc}
\mathrm{i} \sigma_{2} & 0 \\
0 & \mathrm{i} \sigma_{2}
\end{array}\right) \text {. }
$$

Analogously to the previous bound state, we checked that the VEVs indeed give rise to the breaking pattern

$$
\mathrm{U}(2)^{5} \times \mathrm{U}(4)^{2} \rightarrow \mathrm{U}(2) \stackrel{\text { Orientifolding }}{\longrightarrow} \mathrm{USp}(2) .
$$

As before, the oriented model contains 64 real scalars in adjoints of $\mathrm{U}(2)$.

Higgsing — the full quiver. Having built suitable bound states, we are now ready to construct the breaking pattern of the full $\mathrm{dP}_{5}$-quiver. We require two copies of each bound state $F_{a}, F_{b}$ to ensure the existence of two new $\mathrm{U}(2)$ factors. With these guidelines, we 


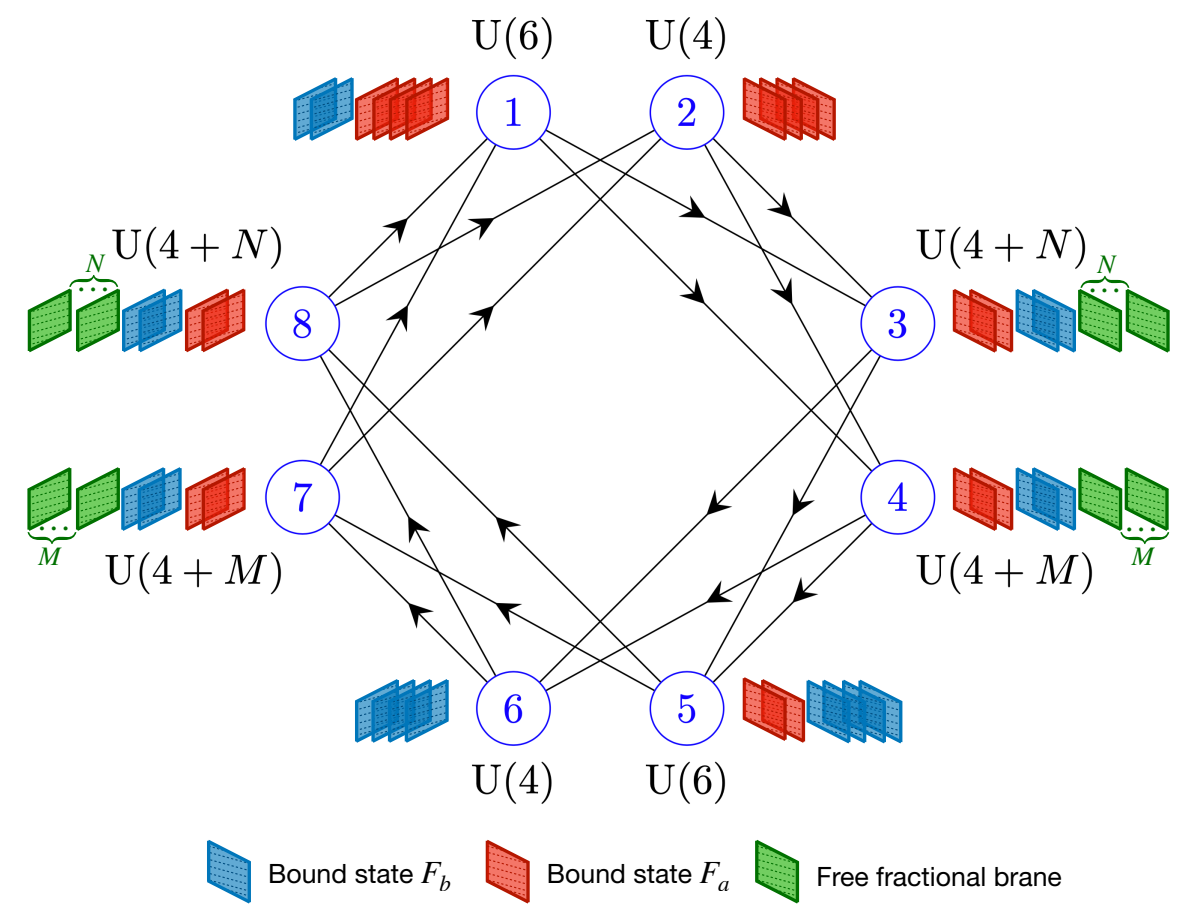

Figure 6. Model I quiver diagram $\mathrm{dP}_{5}$ with the bound state $2 F_{a}$ indicated in red and $2 F_{b}$ in blue. The green fractional branes do not participate in any bound state.

construct a quiver specified by $\left(2 F_{a}, N F_{3}, M F_{4}, 2 F_{b}, M F_{7}, N F_{8}\right)$ corresponding to multiplicities $(6,4,4+N, 4+M, 6,4,4+M, 4+N)$ of fractional branes in the exceptional collection $\left\{F_{1}, \ldots, F_{8}\right\}$ as illustrated in figure 6 . The choice $N=3, M=1$ has already been discussed in [23]. The bound state $2 F_{a}$ is depicted in red in figure 6 , whereas $2 F_{b}$ in blue.

The breaking of the full $\mathrm{dP}_{5}$-quiver is achieved by embedding the VEVs for the bound states $2 F_{b}$ and $2 F_{a}$ into representations of the full quiver. To this end, we define the VEVs

$$
\begin{aligned}
& \left\langle X_{13}\right\rangle=\left(\begin{array}{ccc}
a_{1} & 0 & \cdots \\
0 & a_{2} & \cdots \\
0 & a_{3} & \cdots
\end{array}\right), \quad\left\langle X_{14}\right\rangle=\left(\begin{array}{ccc}
b_{1} & 0 & \cdots \\
0 & b_{2} & \cdots \\
0 & b_{3} & \cdots
\end{array}\right), \\
& \left\langle X_{23}\right\rangle=\left(\begin{array}{ccc}
0 & c_{1} & \cdots \\
0 & c_{2} & \cdots
\end{array}\right), \quad\left\langle X_{24}\right\rangle=\left(\begin{array}{lll}
0 & d_{1} & \cdots \\
0 & d_{2} & \cdots
\end{array}\right), \\
& \left\langle X_{35}^{T}\right\rangle=\left(\begin{array}{ccc}
x_{1} & 0 & \cdots \\
x_{2} & 0 & \cdots \\
0 & x_{3} & \cdots
\end{array}\right), \quad\left\langle X_{45}^{T}\right\rangle=\left(\begin{array}{ccc}
z_{1} & 0 & \cdots \\
z_{2} & 0 & \cdots \\
0 & z_{3} & \cdots
\end{array}\right), \\
& \left\langle X_{36}^{T}\right\rangle=\left(\begin{array}{lll}
y_{1} & 0 & \cdots \\
y_{2} & 0 & \cdots
\end{array}\right), \quad\left\langle X_{46}^{T}\right\rangle=\left(\begin{array}{lll}
w_{1} & 0 & \cdots \\
w_{2} & 0 & \cdots
\end{array}\right)
\end{aligned}
$$

where all entries are $2 \times 2$-matrices. The $\cdots$ represent $N$ zeros for the matrices on the left side and $M$ zeros for the matrices on the right. The remaining VEVs for $\left\langle X_{81}\right\rangle$ etc. are 


\section{Higgsed quiver}

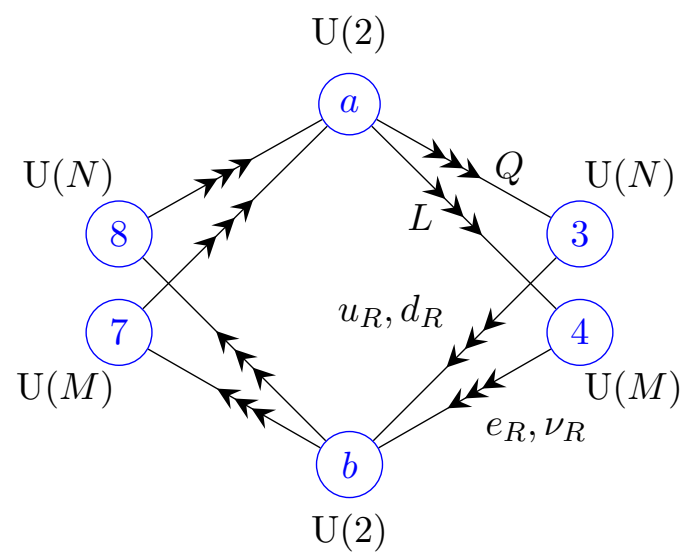

Figure 7. Higgsed quiver diagram $\mathrm{dP}_{5}$ showing only the chiral matter spectrum.

fixed via the orientifold condition (2.29) with

$$
\gamma_{1}=\gamma_{5}=\mathrm{i} \sigma_{2} \otimes \mathbb{1}_{3 \times 3}, \quad \gamma_{2}=\gamma_{6}=\mathrm{i} \sigma_{2} \otimes \mathbb{1}_{2 \times 2} .
$$

With our previous arguments, the breaking pattern is equivalently achieved by the choice of [23] given by

$$
\begin{array}{llll}
\left.\left\langle X_{13}\right\rangle\right|_{a_{3}=0}, & \left.\left\langle X_{14}\right\rangle\right|_{b_{2}=0}, & \left.\left\langle X_{23}\right\rangle\right|_{c_{2}=0}, & \left.\left\langle X_{24}\right\rangle\right|_{d_{1}=0}, \\
\left.\left\langle X_{35}^{T}\right\rangle\right|_{x_{2}=0}, & \left.\left\langle X_{45}^{T}\right\rangle\right|_{z_{1}=0}, & \left.\left\langle X_{36}^{T}\right\rangle\right|_{y_{2}=0}, & \left.\left\langle X_{46}^{T}\right\rangle\right|_{w_{1}=0}
\end{array}
$$

We claim that both of these choices of VEVs break the original gauge group $G$ as

$$
G=(\mathrm{U}(6) \times \mathrm{U}(4+N) \times \mathrm{U}(4+M) \times \mathrm{U}(4))^{2} \rightarrow H=(\mathrm{U}(2) \times \mathrm{U}(N) \times \mathrm{U}(M))^{2} .
$$

The resulting quiver is shown in figure 7 . Let us try to see this more explicitly applying the observations from table 2. First notice that each $2 \times 2$ matrix breaks a $\mathrm{U}(2)^{2}$ to a diagonal $\mathrm{U}(2)$. Then $\left\langle X_{13}\right\rangle$ breaks the original $\mathrm{U}(6) \times \mathrm{U}(4+N)$ of nodes 1 and 3 to a $\mathrm{U}(2)^{3} \times \mathrm{U}(N)$ with the first two $\mathrm{U}(2)$ 's corresponding to diagonal $\mathrm{U}(2)$ 's. Similarly $\left\langle X_{14}\right\rangle$ breaks $\mathrm{U}(6) \times \mathrm{U}(4+M)$ of nodes 1 and 4 to $\mathrm{U}(2)^{3} \times \mathrm{U}(M)$ with the first and third $\mathrm{U}(2)$ being diagonal (and the first combining with the first of the previous $\left.\mathrm{U}(2)^{3}\right)$. Going through all the breaking patterns from these VEVs we can see that there are only two independent $\mathrm{U}(2)$ 's surviving associated with the bound states $2 F_{b}$ and $2 F_{a}$. In terms of the full quiver, the former one is a diagonal combination of the first $\mathrm{U}(2)$ on nodes $1,3,4,5,6$ and the second of nodes 5 and 6 . The second $\mathrm{U}(2)$ is a combination of the second and third of the U(2)'s of node 1 , the first and second $\mathrm{U}(2)$ of node 2 the second $\mathrm{U}(2)$ of nodes 3,4 and the third of node 6 .

\subsection{Matter spectrum for model I}

Chiral matter. Regarding the matter spectrum we have to concentrate on the decomposition of the original states. For the fields connected to node 3 , the breaking pat- 
tern decomposes bi-fundamentals of $\mathrm{U}(6) \times \mathrm{U}(4+N)$ into suitable representations under $\mathrm{U}(2) \times \mathrm{U}(2) \times \mathrm{U}(N)$ such that

$$
\begin{array}{llll}
X_{13}: & (\mathbf{6}, \overline{\mathbf{4}+\mathbf{N}}) \rightarrow(\mathbf{2}, \mathbf{1}, \overline{\mathbf{N}})+2(\mathbf{1}, \mathbf{2}, \overline{\mathbf{N}})+\ldots \\
X_{23}: & (\mathbf{4}, \overline{\mathbf{4}+\mathbf{N}}) \rightarrow 2(\mathbf{1}, \mathbf{2}, \overline{\mathbf{N}})+\ldots \\
X_{35}: & (\mathbf{4}+\mathbf{N}, \overline{\mathbf{4}}) \longrightarrow 2(\mathbf{2}, \mathbf{1}, \mathbf{N})+\ldots \\
X_{36}: & (\mathbf{4}+\mathbf{N}, \overline{\mathbf{6}}) \longrightarrow 2(\mathbf{2}, \mathbf{1}, \mathbf{N})+(\mathbf{1}, \mathbf{2}, \mathbf{N})+\ldots
\end{array}
$$

Here, the $\ldots$ are additional fields charged under $\mathrm{U}(2)$, but not under $\mathrm{U}(N)$. Altogether, the matter content between $\mathrm{U}(N)$ and any of the $\mathrm{U}(2)$ 's corresponds to

$$
\begin{aligned}
& 3((\mathbf{2}, \mathbf{1}, \mathbf{N})+(\mathbf{1}, \mathbf{2}, \overline{\mathbf{N}})) \quad \longrightarrow \quad 3 \text { chiral families } \\
& (\mathbf{2}, \mathbf{1}, \mathbf{N})+(\mathbf{2}, \mathbf{1}, \overline{\mathbf{N}}) \quad \longrightarrow \quad \text { vector-like pair }, \\
& (\mathbf{1}, \mathbf{2}, \mathbf{N})+(\mathbf{1}, \mathbf{2}, \overline{\mathbf{N}}) \quad \longrightarrow \quad \text { vector-like pair . }
\end{aligned}
$$

This means there are three chiral families as indicated on the left of figure 7. On top of that, we find pairs of vector-like states that can pair up to get a mass through superpotential couplings.

Another way to obtain the chiral matter spectrum is using the symmetrised intersection formula (2.9) for fractional branes [71]. Indeed, we obtain for the intersection matrix of fractional branes $\left\{F_{a}, F_{3}, F_{4}, F_{b}, F_{7}, F_{8}\right\}$

$$
\chi_{-}\left(F_{i}, F_{j}\right)=\left(\begin{array}{cccccc}
0 & 3 & 3 & 0 & -3 & -3 \\
-3 & 0 & 0 & 3 & 0 & 0 \\
-3 & 0 & 0 & 3 & 0 & 0 \\
0 & -3 & -3 & 0 & 3 & 3 \\
3 & 0 & 0 & -3 & 0 & 0 \\
3 & 0 & 0 & -3 & 0 & 0
\end{array}\right) .
$$

The resulting 3 family chiral spectrum agrees with our field theory expectation on the left of figure 7 .

To summarise, we find a quiver with three chiral families of $(\mathrm{U}(2) \times \mathrm{U}(N) \times \mathrm{U}(M))^{2}$ which upon orientifolding is the covering quiver of a three-family left-right symmetric model $\mathrm{USp}(2)_{L} \times \mathrm{USp}(2)_{R} \times \mathrm{U}(N) \times \mathrm{U}(M)$.

Non-chiral matter. To find the excess non-chiral matter, we count the number of Goldstone modes

$$
\operatorname{dim}(G / H)=160+16(N+M) .
$$

The number of complex scalar fields in bi-fundamentals in the original quiver is

$$
N_{\text {chiral }}=320+40(N+M)
$$

while after Higgsing the three chiral families amount to

$$
N_{\text {chiral }}^{\text {Higgsed }}=24(N+M)
$$


complex scalars. The Goldstone modes are being eaten by the massive gauge potentials to account for the longitudinal polarisation. In addition, the new massive vector multiplet absorbs one extra real massive scalar so that the number of (potentially light) complex scalars is

$$
N_{\text {chiral }}-\operatorname{dim}(G / H)=160+24(N+M)=160+N_{\text {chiral }}^{\text {Higgsed }} .
$$

As expected, the massless chiral spectrum is specified as outlined in the preceding section. The remaining 160 complex scalars are either in adjoints of a single $\mathrm{U}(2)$ or in bi-fundamental representations between the two $\mathrm{U}(2)$ factors. Indeed, we expect to find for each of the two bound states four adjoints so that $160-64=96$ complex scalars remain as bi-fundamentals combining into vector-like pairs. However, we also need non-chiral ${ }^{12}$ matter states between $\mathrm{U}(2)_{R}$ and $\mathrm{U}(M=1)$ to break the left-right symmetric model down to the SM gauge group.

Extended model I quiver. This is achieved by constructing a new bound state of fractional branes generating yet another $\mathrm{U}(2)$. The Higgses between the corresponding $\mathrm{U}(2)$ and $\mathrm{U}(M)$ are obtained by replacing (among others) $\mathrm{U}(4+N)$ and $\mathrm{U}(4+M)$ by $\mathrm{U}(10+N)$ and $\mathrm{U}(8+M)$. In this way, we obtain $\operatorname{dim}(G / H) \supset 32 M+40 M$ which is not symmetric in $N$ and $M$. We summarised the necessary choice of VEVs in appendix C.2. The counting of degrees of freedom leads to

$$
\begin{aligned}
G & =(\mathrm{U}(12) \times \mathrm{U}(10+N) \times \mathrm{U}(8+M) \times \mathrm{U}(8))^{2}, \\
H & =(\mathrm{U}(2) \times \mathrm{U}(N) \times \mathrm{U}(M))^{2}, \\
\operatorname{dim}(G / H) & =736+32 M+40 N, \\
N_{\text {chiral }} & =1440+80(N+M), \\
N_{\text {chiral }}^{\text {Higgse }} & =24(N+M), \\
N_{\text {chiral }}-\operatorname{dim}(G / H) & =696+16(N+M)+N_{\text {chiral }}^{\text {Higgsed }}+8 M+8 .
\end{aligned}
$$

We expect the $696+16(N+M)$ fields ${ }^{13}$ to gain masses. According to [23], this is achieved by looking at superpotentials with quartic and octic terms.

Ultimately, the unoriented quiver is the one in figure 8. The massless content consists of the chiral families of the MSSM plus two non-chiral pairs between $\mathrm{U}(2)_{R}$ and $\mathrm{U}(M)$ (additional Higgses to break $\mathrm{U}(2)_{R} \times \mathrm{U}(M=1) \rightarrow \mathrm{U}(1)$ ), two non-chiral pairs charged under $\mathrm{U}(2)_{R} \times \mathrm{U}(2)_{L}$ (the Higgs fields $\left.H_{u}, H_{d}\right)$ and right-handed neutrinos $\nu_{R}$. Secondly, there are two additional gauge bosons associated to $\mathrm{U}(1)_{\mathrm{B}}$ (left node) and $\mathrm{U}(1)_{\mathrm{L}}$ (right node). One combination of U(1)'s is anomalous with the corresponding gauge field gaining a Stückelberg mass through the Green-Schwarz mechanism. The non-anomalous combination $\mathrm{U}(1)_{\mathrm{B}-\mathrm{L}}$ together with $\mathrm{SU}(2)_{R}$ is broken to the hypercharge $\mathrm{U}(1)_{\mathrm{Y}}$ via conventional Higgsing which leads to the quiver depicted in figure 1 .

\footnotetext{
${ }^{12}$ In fact, it has been argued in [23] that spectrum is completely chiral. That is, all vector-like states become massive through superpotential couplings.

${ }^{13}$ We find from the analysis of bound states that $400+32$ complex scalars are in adjoints at the $\mathrm{U}(2)$ factors so that $696-(400+32)=264$ states remain between the two U(2)'s which need to get massive.
} 


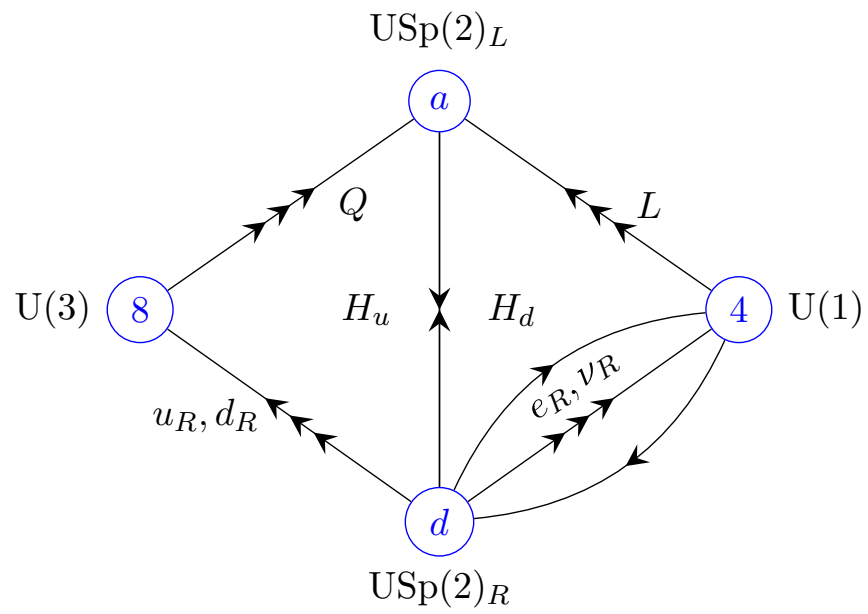

Figure 8. Orientifolded and Higgsed quiver diagram $\mathrm{dP}_{5}$ for the larger version of model I. After identifying $\mathrm{USp}(2) \cong \mathrm{SU}(2)$, this mirrors a supersymmetric version of the left-right symmetric model.

\section{$2.7 \quad F$ - and $D$-term flatness}

For simplicity, we discuss the D- and F-flatness conditions for the small version of Model I in this section. Details on the extended Model I can be found in appendix C.2.

$D$-term conditions. For each non-abelian factor $\mathrm{SU}\left(N_{i}\right)$ or each node $i$ in the quiver, there are D-flatness conditions for each generator of the form

$$
D_{i}^{a}=X_{i j} T_{j k}^{a} X_{k i}^{\dagger}-Y_{i j} T_{j k}^{a} Y_{k i}^{\dagger}=0
$$

with ingoing arrows $X_{i j}$ and outgoing $Y_{i j}$ (for details see appendix C.1). The D-term conditions associated with nodes on the top or bottom in figure 6 are trivially satisfied since the legs to left and right are identical due to the orientifold symmetry. The only non-trivial conditions are given by

$$
\left|a_{1}\right|^{2}+\left|a_{2}\right|^{2}+\left|a_{3}\right|^{2}+\left|c_{1}\right|^{2}+\left|c_{2}\right|^{2}-\left|x_{1}\right|^{2}-\left|x_{2}\right|^{2}-\left|x_{3}\right|^{2}-\left|y_{1}\right|^{2}-\left|y_{2}\right|^{2}=0
$$

which can be solved by choosing

$$
\left|a_{1}\right|^{2}=\left|x_{1}\right|^{2}+\left|x_{2}\right|^{2}+\left|y_{1}\right|^{2}+\left|y_{2}\right|^{2}, \quad\left|x_{3}\right|^{2}=\left|a_{2}\right|^{2}+\left|a_{3}\right|^{2}+\left|c_{1}\right|^{2}+\left|c_{2}\right|^{2} .
$$

Furthermore, we obtain

$$
\left|b_{1}\right|^{2}+\left|b_{2}\right|^{2}+\left|b_{3}\right|^{2}+\left|d_{1}\right|^{2}+\left|d_{2}\right|^{2}-\left|z_{1}\right|^{2}-\left|z_{2}\right|^{2}-\left|z_{3}\right|^{2}-\left|w_{1}\right|^{2}-\left|w_{2}\right|^{2}=0
$$

which is solved for

$$
\left|b_{1}\right|^{2}=\left|z_{1}\right|^{2}+\left|z_{2}\right|^{2}+\left|w_{1}\right|^{2}+\left|w_{2}\right|^{2}, \quad\left|z_{3}\right|^{2}=\left|b_{2}\right|^{2}+\left|b_{3}\right|^{2}+\left|d_{1}\right|^{2}+\left|d_{2}\right|^{2} .
$$

All other D-flatness conditions vanish by the symmetry of the quiver. For the choice (2.52) of VEVs, these conditions are equivalent to the ones given in [23]. 
For the two $\mathrm{U}(2)$ groups and the second $\mathrm{U}(4+M)$ and $\mathrm{U}(4+N)$ the $\mathbb{Z}_{2}$ symmetry of the quiver implies automatic cancellation. We have 4 real conditions leaving 36 unfixed real parameters out of the original 20 complex parameters $a_{i}, b_{i}, c_{j}, d_{j}, x_{i}, z_{i}, y_{j}, w_{j}$. The other 20 parameters coming from the other half of the quiver are fixed by keeping the $\mathbb{Z}_{2}$ symmetry of the quiver to be orientifolded.

Next, we consider the D-term conditions of the abelian $\mathrm{U}(1)$ 's inside $\mathrm{U}\left(N_{i}\right)$, that is,

$$
D_{i}=Q_{i}^{(a b)}\left|X_{a b}\right|^{2}=Q_{i}^{(a b)} \operatorname{Tr}\left(X_{a b}^{\dagger} X_{a b}\right)=0 .
$$

Explicitly, we find

$$
\begin{aligned}
& D_{1}=D_{2}=D_{5}=D_{6}=0 \\
& D_{8}=-D_{3}, \quad D_{7}=-D_{8}, \\
& D_{3}=-2\left(\left|a_{1}\right|^{2}+\left|a_{2}\right|^{2}+\left|a_{3}\right|^{2}+\left|c_{1}\right|^{2}+\left|c_{2}\right|^{2}-\left|x_{1}\right|^{2}-\left|x_{2}\right|^{2}-\left|x_{3}\right|^{2}-\left|y_{1}\right|^{2}-\left|y_{2}\right|^{2}\right), \\
& D_{4}=-2\left(\left|b_{1}\right|^{2}+\left|b_{2}\right|^{2}+\left|b_{3}\right|^{2}+\left|d_{1}\right|^{2}+\left|d_{2}\right|^{2}-\left|w_{1}\right|^{2}-\left|w_{2}\right|^{2}-\left|z_{1}\right|^{2}-\left|z_{2}\right|^{2}-\left|z_{3}\right|^{2}\right) .
\end{aligned}
$$

There are only two distinguishable non-trivial D-terms $D_{3}$ and $D_{4}$ which are related to $D_{7}$ and $D_{8}$ due to the symmetry of the quiver. These conditions are trivially satisfied using the non-abelian D-flatness conditions (2.64) and (2.66). This also implies that both D-terms for the two anomalous U(1)'s vanish. Therefore, the two FI parameters $\xi_{7,8}$ are identically zero which immediately sets the model at the singularity.

$\boldsymbol{F}$-term flatness. Finally, we claim that our choice of VEVs is sufficient to ensure $F$-term flatness in the vacuum. The $F$-term conditions are given by

$$
D_{X_{a b}} W=\frac{\partial W}{\partial X_{a b}}+\frac{\partial K}{\partial X_{a b}} W=0 .
$$

They need to be studied together with the dependence of the full superpotential $W$ and Kähler potential on the complex structure moduli $U$. Despite this, we argue that (2.69) can always be satisfied through suitable complex structure deformations along the lines of $[72,82]$.

The argument is the standard that $\mathrm{dP}_{n}$ is defined by $\mathbb{P}^{2}$ with $n$ blow-up points. The automorphism group of $\mathbb{P}^{2}, \operatorname{PGL}(3, n)$, has $3^{2}-1=8$ parameters. Therefore the $n$ blow-up points for $\mathrm{dP}_{n}$ are determine by $2 n-8$ complex parameters and the number of complex structure deformations is encoded in

$$
\operatorname{dim}\left(H^{1}\left(\mathrm{dP}_{n}, T \mathrm{dP}_{n}\right)\right)= \begin{cases}2 n-8 & 5 \leq n \leq 8 \\ 0 & n \leq 4 .\end{cases}
$$

For $n=5$, there are 2 complex structure parameters that appear in the superpotential

$$
\begin{aligned}
W_{Q}= & \alpha_{1} X_{13} X_{35} X_{58} X_{81}-\alpha_{2} X_{14} X_{46} X_{68} X_{81}+\alpha_{3} X_{14} X_{45} X_{57} X_{71}-\alpha_{4} X_{13} X_{36} X_{67} X_{71}+ \\
& \alpha_{5} X_{24} X_{46} X_{67} X_{72}-\alpha_{6} X_{23} X_{35} X_{57} X_{72}+\alpha_{7} X_{23} X_{36} X_{68} X_{82}-\alpha_{8} X_{24} X_{45} X_{58} X_{82}+ \\
& \beta_{1} X_{13} X_{35} X_{57} X_{71}-\beta_{2} X_{14} X_{46} X_{67} X_{71}+\beta_{3} X_{14} X_{45} X_{58} X_{81}-\beta_{4} X_{13} X_{36} X_{68} X_{81}+ \\
& \beta_{5} X_{24} X_{46} X_{68} X_{82}-\beta_{6} X_{23} X_{35} X_{58} X_{82}+\beta_{7} X_{23} X_{36} X_{67} X_{72}-\beta_{8} X_{24} X_{45} X_{57} X_{72}
\end{aligned}
$$


with the coefficients $\alpha_{i}, \beta_{i}$ functions of complex structure moduli $U$. It has been suggested in $[72,82]$ that tuning the complex structure parameters can allow to fix all $F$-term conditions. For a globally embedded model the situation is more involved since we have to consider all the fields in the full superpotential. Hence, we will argue that once we have a global embedding of the quiver model, there is plenty of freedom to satisfy the $F$-term conditions $D_{S, U, X} W=0$ coming from the rich structure provided by the fluxes and also the 18 free complex parameters of our ansatz (2.50). This will be discussed further in section 5 .

\section{Calabi-Yau threefolds with diagonal $\mathrm{dP}_{5}$ divisors}

\subsection{Embedding the local model in a compact CY threefold}

We aim to embed the local model described in section 2 into a global $\mathrm{CY}$ orientifold compactification. Of course, the first feature that the compact CY should have is the presence of a $\mathrm{dP}_{5}$ singularity. This singularity is obtained by taking a limit from a smooth $\mathrm{CY}$ with a $\mathrm{dP}_{5}$ divisor. The singularity is generated when the volume of the $\mathrm{dP}_{5}$ divisor goes to zero.

Since we want a global model with moduli that are stabilised at a dS minimum, we need to ask for other properties of the CY. In particular the desired features of the model are the following:

1. As we said, we need CY threefolds with $\mathrm{dP}_{5}$ divisors in order to embed the local model of section 2. In particular we need the $\mathrm{dP}_{5}$ to be 'diagonal', such that shrinking it to a point does not force other divisors to shrink (generating a different singularity with respect to the one considered in the local model).

2. There must be an involution such that the $\mathrm{dP}_{5}$ divisor is transversely invariant and that it intersects the O7-plane (like in the local model, see also appendix D). ${ }^{14}$

3. It is desirable to possibly have $O 7$-planes with large $\chi(O 7)$ to have large negative $D 3$ charge. This would allow to easily satisfy the $D 3$-tadpole cancellation condition.

4. In order to have a T-brane uplifting to de Sitter [22,35], we also need the involution to be such that some D7-branes wrap large (in the LVS sense) divisors. On the other hand, if the dS uplift is realised via anti- $D 3$ brane, we may want an involution that generates $O 3$-planes at some appropriate locations [83].

5. One needs to check the tadpole/anomaly cancellation conditions and that the nonperturbative superpotential contribution is generated. In particular, we require the presence of at least one additional diagonal $\mathrm{dP}_{n}$ to support the LVS construction $[37,84]$.

\footnotetext{
${ }^{14}$ A systematic formalism to constructing CY orientifolds with fluxed branes wrapping shrinkable del Pezzo divisors has been laid out in [7]. In addition, it contains a classification of involutions of del Pezzo surfaces which is highly relevant for our considerations.
} 


\subsection{Calabi-Yau threefolds with diagonal $\mathrm{dP}_{n}$}

Requirements for having a diagonal $\mathbf{d P}_{\boldsymbol{n}}$ divisor. As mentioned above, we need to search for Calabi Yau threefolds $X$ which have at least one (diagonal) $\mathrm{dP}_{5}$ divisor. We will work with $\mathrm{CYs}$ that are embedded into toric ambient spaces. Here $\mathrm{dP}_{5}$ divisors are usually obtained by the so-called "coordinate divisors" $D_{i}$ which are defined by intersecting the CY equation with the loci given by setting a toric coordinate to zero: $x_{i}=0$. This is sufficient for capturing the del Pezzo surfaces in CYs $X$ with $h^{1,1}(X)=h^{1,1}(A)$, where $A$ is the ambient space. We only consider such spaces. ${ }^{15}$

A del Pezzo divisor must satisfy the following topological conditions:

$$
\int_{X} D_{s}^{3}=k_{s s s}>0, \quad \int_{X} D_{s}^{2} D_{i} \leq 0 \quad \forall i \neq s .
$$

Here $k_{s s s}=9-n$ for a $\mathrm{dP}_{n}$ divisor is the degree of $\mathrm{dP}_{n}$. We moreover look for divisors $D_{s}$ that satisfy the following 'diagonality' condition [85]

$$
k_{s s s} k_{s i j}=k_{s s i} k_{s s j} \quad \forall i, j .
$$

If this condition is satisfied, then the volume of the four-cycle $D_{s}$ is a complete-square:

$$
\tau_{s}=\frac{1}{2} k_{s i j} t^{i} t^{j}=\frac{1}{2 k_{s s s}} k_{s s i} k_{s s j} t^{i} t^{j}=\frac{1}{2 k_{s s s}}\left(k_{s s i} t^{i}\right)^{2},
$$

where we sum over $i, j$ but not over $s$. One can then shrink the del Pezzo divisor to a point along one direction of the Kähler moduli space, simply by setting to zero the combination of the $t_{i}$ that appear on the r.h.s.

A conjecture for diagonal del Pezzo $d \boldsymbol{P}_{n}$ with $\mathbf{1} \leq \boldsymbol{n} \leq \mathbf{5}$. As explained in appendix A, we performed a scan over hypersurface CY threefolds obtained from polytope triangulations listed in [86]. ${ }^{16}$ For CYs in this database, we found that the diagonality condition (3.2) could never be satisfied for the $\mathrm{dP}_{5}$ divisors. ${ }^{17}$

The analysis made in appendix A led us to the following conjecture:

"The Calabi Yau threefolds arising from the four-dimensional reflexive polytopes listed in the Kreuzer-Skarke database do not exhibit a 'diagonal' del Pezzo divisor $\mathrm{dP}_{n}$ for $1 \leq n \leq 5$, in the sense of satisfying the eq. (3.2)."

Using the topological data of the CY threefolds collected in the AGHJN-database [86], we have checked this conjecture to hold for $1 \leq h^{1,1}(X) \leq 5$. It would be interesting to explore

\footnotetext{
${ }^{15}$ In fact, in these cases the divisors are given by the intersection of a divisor of $A$ with the equation defining $X$; the rigid divisors (like the del Pezzo divisors) are among the coordinates divisors.

${ }^{16}$ These were based on the four-dimensional reflexive polytopes listed in the Kreuzer-Skarke (KS) database [31] with $h^{1,1} \leq 6$.

${ }^{17}$ For example, most of the times the volume of $\mathrm{dP}_{5}$ four-cycle takes the form $\tau_{\mathrm{dP}_{5}}=\left(\sum_{i} a_{i} t^{i}\right)\left(\sum_{j} b_{j} t^{j}\right)$ for some $i \neq j$. Now setting one of the two linear combinations to zero, makes the size of the $\mathrm{dP}_{5}$ go to zero like $t$ instead of $t^{2}$. This is a signal that the divisor has not shrunk to a point, but rather to a curve. In order to shrink it to a point one needs to set to zero both combinations of the $t_{i}$ 's. This is what we call a 'non-diagonal' del Pezzo.
} 
its validity further or find a counter example against our claim. To begin with, we scanned further 300.000 geometries with $6 \leq h^{(1,1)} \leq 40$ using the software package CYTools [87] providing evidence that the conjecture might even hold at large values of $h^{(1,1)}$.

Due to this result, we were forced to explore CYs embedded in toric ambient spaces of dimension larger than 4 . We have actually been able to construct several CYs (see appendix B) that are given by two equations in a 5 dimensional toric ambient space. These have diagonal $\mathrm{dP}_{5}$ divisors.

$\mathbf{d P}_{5}$ surface as a bi-quadric in $\mathbb{P}^{4}$. As just mentioned, it is possible to construct

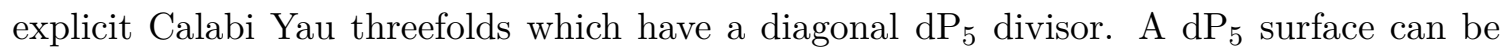
represented by a bi-quadric in $\mathbb{P}^{4}$ which is given by the following toric data,

\begin{tabular}{|c|c||ccccc|}
\hline$H Y_{1}$ & $H Y_{2}$ & $x_{1}$ & $x_{2}$ & $x_{3}$ & $x_{4}$ & $x_{5}$ \\
\hline 2 & 2 & 1 & 1 & 1 & 1 & 1 \\
\hline
\end{tabular}

with the SR ideal being given as $\left\{x_{1} x_{2} x_{3} x_{4} x_{5}\right\}$. Using cohomCalg [88, 89] it is easy to confirm that this has the topology of a $\mathrm{dP}_{5}$ surface.

We then expect to find $\mathrm{dP}_{5}$ divisors in CYs that are complete intersections of two equations with a five-dimensional toric space. Setting one coordinate to zero and properly gauge fixing all the $\mathbb{C}^{*}$ action except one, we should end up with the surface (3.4) (we will see this explicitly in the concrete model in section 4 .

\section{Global embedding of $\mathrm{dP}_{5}$ model}

We have worked out few examples of complete intersection CY's (CICY) that have a $\mathrm{dP}_{5}$ singularity in some region of their moduli space. In this section we analyse in detail one of them, in order to provide an example of global embedding of our local model. The other CY's can be found in appendix B.

\subsection{Geometric data}

Here we consider the following CICY threefold $X$ which has three diagonal $\mathrm{dP}_{5}$ divisors. As observed above, it is given by two equations intersecting a five dimensional toric space. The toric data for such a CICY threefold is summarised in table 3 with the SR-ideal being given $\operatorname{as}^{18}$

$$
\mathrm{SR}=\left\{x_{2} x_{3}, x_{2} x_{4}, x_{2} x_{9}, x_{3} x_{4}, x_{4} x_{7}, x_{1} x_{7} x_{9}, x_{3} x_{5} x_{6} x_{8}, x_{1} x_{5} x_{6} x_{7} x_{8}, x_{1} x_{5} x_{6} x_{8} x_{9}\right\} .
$$

This CY threefold has the Hodge numbers $\left(h^{2,1}, h^{1,1}\right)=(52,4)$ and Euler characteristic $\chi=-96$. The first two columns of table 3 provide the degrees of the polynomial equations defining the CY threefold $X$.

An integral basis of $H^{1,1}(X, \mathbb{Z})$ is given by $\left\{D_{b}, D_{2}, D_{3}, D_{4}\right\}$, where $D_{b} \equiv D_{1}+D_{2}+$ $D_{3}+D_{4}$. In this basis, the intersection form is

$$
I_{3}=4 D_{b}^{3}+4 D_{2}^{3}+4 D_{3}^{3}+4 D_{4}^{3},
$$

\footnotetext{
${ }^{18}$ There are other triangulations giving three diagonal $\mathrm{dP}_{5}$. We took the one where computations are in a simpler form.
} 


\begin{tabular}{|c|c||ccccccccc|}
\hline$H Y_{1}$ & $H Y_{2}$ & $x_{1}$ & $x_{2}$ & $x_{3}$ & $x_{4}$ & $x_{5}$ & $x_{6}$ & $x_{7}$ & $x_{8}$ & $x_{9}$ \\
\hline 4 & 4 & 1 & 0 & 0 & 0 & 2 & 2 & 1 & 1 & 1 \\
2 & 2 & 0 & 1 & 0 & 0 & 1 & 1 & 0 & 0 & 1 \\
2 & 2 & 0 & 0 & 1 & 0 & 1 & 1 & 0 & 1 & 0 \\
2 & 2 & 0 & 0 & 0 & 1 & 1 & 1 & 1 & 0 & 0 \\
\hline & & $\mathrm{NdP}_{17}$ & $\mathrm{dP}_{5}$ & $\mathrm{dP}_{5}$ & $\mathrm{dP}_{5}$ & $\mathrm{SD} 1$ & $\mathrm{SD} 1$ & $\mathrm{SD} 2$ & $\mathrm{SD} 2$ & $\mathrm{SD} 2$ \\
\hline
\end{tabular}

Table 3. Toric data of $X$.

while the second Chern class is

$$
c_{2}(X)=10 D_{b}^{2}+D_{2}^{2}+D_{3}^{2}+D_{4}^{2} .
$$

A detailed divisor analysis using cohomCalg $[88,89]$ shows that the three divisors $D_{2}$, $D_{3}$ and $D_{4}$ are del Pezzo $\mathrm{dP}_{5}$ surfaces while the divisor $D_{1}$ is what we call 'rigid but not del Pezzo' NdP $n$. In addition, the divisors $\left\{D_{5}, \ldots, D_{9}\right\}$ are 'special deformation' type divisors with the following Hodge diamond:

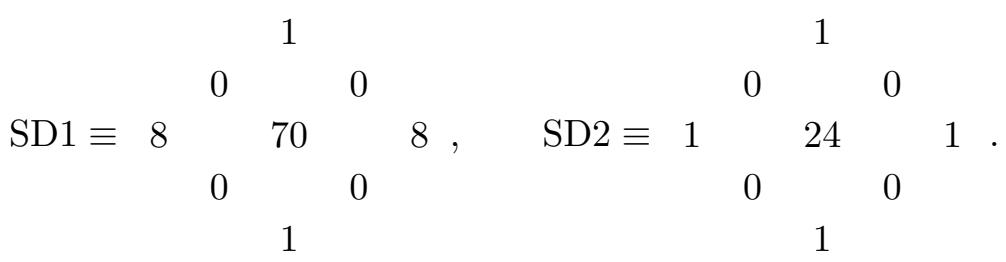

Expanding the Kähler form in the basis $\left\{D_{b}, D_{2}, D_{3}, D_{4}\right\}, J=t_{b} D_{b}+t_{2} D_{2}+t_{3} D_{3}+$ $t_{4} D_{4}$, one obtains the following volumes for the basis divisors

$$
\tau_{i} \equiv \operatorname{vol}\left(D_{i}\right)=\frac{1}{2} \int_{D_{i}} J^{2}=2 t_{i}^{2}, \quad \text { with } \quad i=b, 2,3,4
$$

The volume of the CY threefold is then

$$
\mathcal{V}=\frac{1}{6} \int_{X} J^{3}=\frac{1}{3 \sqrt{2}}\left(\tau_{b}^{3 / 2}-\tau_{2}^{3 / 2}-\tau_{3}^{3 / 2}-\tau_{4}^{3 / 2}\right)
$$

In particular, from this expression we notice that all three $\mathrm{dP}_{5}$ divisors are diagonal. Moreover, the Kähler cone is given by ${ }^{19}$

$$
t_{2}<0, \quad t_{3}<0, \quad t_{4}<0, \quad t_{2}+t_{3}+t_{b}>0, \quad t_{2}+t_{4}+t_{b}>0, \quad t_{3}+t_{4}+t_{b}>0 .
$$

Hence, we can equivalently shrink any of the $\mathrm{dP}_{5}$ to a point-like singularity by squeezing along a single direction. We will make the choice to shrink $D_{2}$, by taking $t_{2} \rightarrow 0$.

\footnotetext{
${ }^{19}$ It has been computed by the union of ambient space Kähler cones relative to triangulations leading to the same intersection form on the CY threefold.
} 


\subsection{Orientifold involution}

We consider the involution

$$
\sigma: \quad x_{5} \mapsto-x_{5}
$$

The CY defining equations that respect this involution can be written as

$$
a^{\lambda} x_{5}^{2}=P_{4,2,2,2}^{\lambda}\left(x_{1}, x_{2}, x_{3}, x_{4}, x_{6}, x_{7}, x_{8}, x_{9}\right), \quad \lambda=1,2
$$

where the r.h.s. does not depend on $x_{5}$.

The fixed point set of the involution is given by the codimension- 1 locus $\left\{x_{5}=0\right\}$. There are no isolated fixed points. We then have a single O7-plane wrapping the divisor $D_{5}=2 D_{b}-D_{2}-D_{3}-D_{4}$. In particular we have $O 7^{3}=D_{5}^{3}=20$ and $\chi(O 7)=88$.

The involution $\sigma$ splits the cohomology groups into eigenspaces, whose dimensions are $h_{ \pm}^{p, q}$, with $h^{p, q}=h_{+}^{p, q}+h_{-}^{p, q}$. For our CICY $X$, it is easy to see that $h_{+}^{1,1}=4$, while $h_{-}^{1,1}=0$. It is less trivial to obtain $h_{ \pm}^{1,2}$. However, we can do it by means of Lefschetz fixed point theorem, after having derived the fixed point set of our involution. For a CY threefold, this theorem says that

$$
2+2\left(h_{+}^{1,1}-h_{-}^{1,1}\right)-2\left(h_{+}^{1,2}-h_{-}^{1,2}-1\right)=\chi\left(O_{\sigma}\right)
$$

with $O_{\sigma}$ the fixed point locus; in our case $O_{\sigma}=O 7$. Combining the relation $h_{+}^{1,2}+h_{-}^{1,2}=$ $h^{1,2}=52$ with (4.8), one obtains $h_{+}^{1,2}=7$ and $h_{-}^{1,2}=45$.

\subsection{Embedding of the local model}

We now focus on the region in $X$ close to the 'diagonal' $\mathrm{dP}_{5}$ divisor $D_{2}$. When we shrink this divisor to zero size, the open patch around it becomes a non-compact $\mathrm{CY}$ with a $\mathrm{dP}_{5}$ singularity. Putting D3-branes on top of the singular point, one obtain a model of D3branes at singularity. We now show that, if we consider the involution (4.6), this model is the same discussed in section 2 and whose involution is discussed from the geometric point of view in appendix D.

We start by taking an open patch close to $x_{2}=0$. Because of the SR-ideal (4.6), we can gauge fix three of the four $\mathbb{C}^{*}$ action in table 3 , setting $x_{3}=1, x_{4}=1$ and $x_{9}=1$. The local CY is then described by

\begin{tabular}{|c|c||cccccc|}
\hline$E q_{1}$ & $E q_{2}$ & $x_{1}$ & $x_{2}$ & $x_{5}$ & $x_{6}$ & $x_{7}$ & $x_{8}$ \\
\hline 2 & 2 & 1 & -1 & 1 & 1 & 1 & 1 \\
\hline
\end{tabular}$\quad$ SR $=\left\{x_{1} x_{5} x_{6} x_{7} x_{8}\right\}$

We immediately see that $x_{2}=0$ is given by two quadratic equations in $\mathbb{P}^{4}$, i.e. it is a $\mathrm{dP}_{5}$ divisor.

It is moreover easy to blow down the $\mathrm{dP}_{5}$ divisor. We obtain a three-fold given by two equations in $\mathbb{C}^{5}$, whose coordinates are $x_{1}, x_{5}, x_{6}, x_{7}, x_{8}$. The $\mathrm{dP}_{5}$ singularity is now located at $x_{1}=x_{5}=x_{6}=x_{7}=x_{8}=0$. Close to this point, the CY defining equations are approximated by the following expressions, where we keep only the quadratic monomials 
discarding the subleading higher order terms: ${ }^{20}$

$$
\begin{array}{ll}
E q_{1}: & x_{1}^{2}=x_{7} x_{8}+Q_{2}\left(x_{1}, x_{5}, x_{6}, x_{7}, x_{8}\right)+\ldots \\
E q_{2}: & x_{1}^{2}=\left(x_{6}+x_{5}\right)\left(x_{6}-x_{5}\right)+R_{2}\left(x_{1}, x_{5}, x_{6}, x_{7}, x_{8}\right)+\ldots
\end{array}
$$

We can match this local CY with the one in appendix D, where we found out the proper involution in the local model to obtain the quiver theory shown in figure 8. The local CY in appendix D is at a special point in the complex structure moduli space, where the singularity becomes a $\mathbb{Z}_{2} \times \mathbb{Z}_{2}$ orbifold of the conifold. The local CY in (4.10) reaches that point by specialising the complex structure of $X$ such that the polynomials $Q_{2}$ and $R_{2}$ are identically zero, i.e.

$$
Q_{2}\left(x_{1}, x_{5}, x_{6}, x_{7}, x_{8}\right) \equiv 0 \quad \text { and } \quad R_{2}\left(x_{1}, x_{5}, x_{6}, x_{7}, x_{8}\right) \equiv 0 .
$$

When this happens, the defining equations (4.10) become

$$
\left\{\begin{array}{l}
x_{1}^{2}=x_{7} x_{8} \\
x_{1}^{2}=\left(x_{6}+x_{5}\right)\left(x_{6}-x_{5}\right)
\end{array}\right.
$$

that are exactly the equations (D.4) in appendix D with involution (D.5) $(\epsilon=+1)$ after identifying the coordinates as $C=x_{1}, X=x_{7}, Y=x_{8}, Z=x_{6}+x_{5}$ and $W=x_{6}-x_{5}$.

\subsection{Non-perturbative effects}

In order to stabilise the Kähler moduli, one needs that the $\mathrm{dP}_{5}$ divisors at $x_{3}=0$ and $x_{4}=0$ host a non-perturbative effect. This divisors are invariant (but not fixed) under the orientifold involution.

A D3-brane wrapping an invariant divisor $D$ and having zero flux, i.e.

$$
\mathcal{F}_{E 3} \equiv F_{E 3}-\iota_{D}^{*} B=0
$$

gives an $O(1)$ instanton that could generate a non-perturbative term in the superpotential. Here $\iota_{D}^{*}$ is the pullback map from two-forms on $X$ to two-forms on the surface $D$.

Since the $\mathrm{dP}_{5}$ surface is non-spin, the gauge flux $F_{E 3}$ must be half-integral, since it must satisfy the Freed-Witten quantisation condition [32]

$$
F_{E 3}+\frac{c_{1}(D)}{2} \in H^{2}(D, \mathbb{Z})
$$

In particular, in the present case $c_{1}(S)=-\iota_{S}^{*} S$ with $S=D_{3}, D_{4}$. In order to have zero flux $\mathcal{F}_{E 3}$ one needs a B-field such that [90]

$$
\iota_{S}^{*} B=\frac{\iota_{S}^{*} S}{2}
$$

up to an integral two-form.

\footnotetext{
${ }^{20}$ One can check that these equations can be completed by adding to each monomial the proper factors of $x_{3}, x_{4}, x_{9}$ to make it of degrees in table 3 .
} 
On the other hand, if one takes zero B-field, $B=0$, then an $O(1)$ E3-instanton is not allowed on the non-spin surface. However, a rank two instanton can be present [91]. Such a D3-brane supports a vector bundle $\mathcal{E}$ of rank two. This configuration is invariant under the orientifold involution $\sigma$ when

$$
\sigma^{*}\left(\mathcal{E}^{\vee}\right) \otimes K_{S}=\mathcal{E}
$$

where $K_{S}$ is the canonical line bundle of $S$. A solution to this equation is given by the dual of the holomorphic tangent bundle of $S$ (whose first Chern class is again $-c_{1}(S)=\iota_{S}^{*} S$ ).

\subsection{D-brane setup}

The O7-plane wrapping the $D_{5}$ divisor generates a non-zero D7-tadpole that needs to be cancelled. It is then necessary for the global consistency of the model to introduce D7branes whose total $\mathrm{D} 7$-charge is equal to $-8 D_{5}$. This configuration must be invariant under the orientifold involution (and then the D5-charge is automatically cancelled).

These D7-branes pass through the $\mathrm{dP}_{5}$ singularity, after taking $t_{2} \rightarrow 0$ (in fact, the intersection $D_{2} \cap D_{5}$ is a non-trivial curve). They seem like flavour branes for the fractional D3-branes and one may wonder whether this generates extra chiral states for the theory at the singularity. However a chiral spectrum between D3- and D7-branes would produce an anomalous spectrum (since the local model was anomaly free). However, since a globally consistent model with all tadpoles cancelled must be anomaly free, we expect that the D7-branes do not introduce extra chiral states to the D3-model.

In the following we consider two different D7-brane configurations: the first one is consistent with an $O(1)$ E3-instanton on top of the $\mathrm{dP}_{5}$ divisors at $x_{3}=0$ and $x_{4}=0$ and will consequently have non-zero B-field. The second one is the configurations with the maximal D3-charge but still allowing a T-brane (necessary for dS uplift); it is consistent with the proper structure of zero modes for a rank 2 E3-instanton on the $\mathrm{dP}_{5}$ 's at $x_{3}=0$ and $x_{4}=0$ and one can take zero B-field.

SO(8) D7-brane configuration. We will consider a stack of four D7-branes (plus their four orientifold images) wrapping the locus $x_{5}=0$. This produces an $\mathrm{SO}(8)$ gauge group living on this locus. Remember that $D_{5}=2 D_{b}-D_{2}-D_{3}-D_{4}$.

In order to have an $O(1)$ instanton on $D_{3}$ and $D_{4}$ we choose the B-field as

$$
B=-\frac{D_{3}}{2}-\frac{D_{4}}{2}+\frac{D_{b}}{2} .
$$

The last term is not necessary to make the E3-instanton orientifold invariant, as $\iota_{D_{3,4}}^{*} D_{b}=0$. However, it will be necessary to generate the wanted T-brane.

The following flux $\mathcal{F}$ on each one of the four D7-branes (and $-\sigma^{*} \mathcal{F}$ on the four images) is consistent with flux quantisation:

$$
\mathcal{F}=F-B=\left(n_{b}-\frac{1}{2}\right) D_{b}+\left(n_{2}-\frac{1}{2}\right) D_{2}+n_{3} D_{3}+n_{4} D_{4} \text { with } n_{b}, n_{2}, n_{3}, n_{4} \in \mathbb{Z},
$$

where the symbol $\iota_{D_{5}}^{*}$ is implicit. 
In order for the E3-instanton to generate a non-perturbative term in the superpotential, it should have zero chiral modes ${ }^{21}$ at the intersection with the D7-branes, i.e.

$$
0=\int_{D 7 \cap E 3} \mathcal{F}-\mathcal{F}_{E 3}=D_{5} \cdot D_{\kappa} \cdot \mathcal{F}=-D_{\kappa}^{3} n_{\kappa}=-4 n_{\kappa} \quad \text { with } \kappa=3,4 .
$$

Hence the flux on the D7-branes reduces to

$$
\mathcal{F}=F-B=\left(n_{b}-\frac{1}{2}\right) D_{b}+\left(n_{2}-\frac{1}{2}\right) D_{2} \quad \text { with } \quad n_{b}, n_{2} \in \mathbb{Z} .
$$

This breaks the $\mathrm{SO}(8)$ gauge group to $\mathrm{U}(4)$ (the diagonal $\mathrm{U}(1)$ is actually massive due to a Stückelberg mechanism) and it generates the following FI-term:

$$
\begin{aligned}
\xi_{D 7} & =\frac{1}{4 \pi \mathcal{V}} \int_{D 7} \mathcal{F} \wedge J=\frac{1}{4 \pi \mathcal{V}} D_{5} \cdot \mathcal{F} \cdot\left(t_{b} D_{b}+t_{2} D_{2}+t_{3} D_{3}+t_{4} D_{4}\right) \\
& =\frac{1}{4 \pi \mathcal{V}}\left\{4\left(2 n_{b}-1\right) t_{b}-2\left(2 n_{2}-1\right) t_{2}\right\} \stackrel{t_{2} \rightarrow 0}{\longrightarrow} \frac{1}{\pi \mathcal{V}}\left(2 n_{b}-1\right) t_{b} \simeq \frac{1}{\pi \mathcal{V}^{2 / 3}}\left(\frac{3}{2}\right)^{1 / 3}\left(2 n_{b}-1\right)
\end{aligned}
$$

This FI-term is non-zero. This implies that a non-zero VEV must be switched on for the adjoint complex scalar $\Phi$ living on the D7-brane stack. In particular we will consider a T-brane background $[8,33,34,93]$. For more detail on what we need in this context, see section 3.4 of [22].

Under the breaking of $\mathrm{SO}(8)$ to $\mathrm{U}(4)$ (due to non-zero $\mathcal{F}$ ), the adjoint representation of $\mathrm{SO}(8)$ is broken as:

$$
\mathbf{2 8} \rightarrow \mathbf{1 6}_{0} \oplus \mathbf{6}_{+2} \oplus \mathbf{6}_{-2},
$$

where $\mathbf{R}_{q}$ is in the representation $\mathbf{R}$ for SU(4) and has charge $q$ with respect to the diagonal $\mathrm{U}(1)$. Here $\mathbf{1 6}_{0}=\mathbf{1 5}_{0} \oplus \mathbf{1}_{0}$ is the reducible adjoint representation of $\mathrm{U}(4)$. According to (4.20), the scalar field $\Phi$ can be written as: ${ }^{22}$

$$
\Phi=\left(\begin{array}{cc}
\phi_{1 \mathbf{6}_{0}} & \phi_{\mathbf{6}_{+2}} \\
\phi_{\mathbf{6}_{-2}} & -\phi_{\mathbf{1 6}_{0}}^{T}
\end{array}\right) .
$$

The first four rows (and columns) refer to the four D7-branes, while rows (and columns) from the fifth to the eighth refer to their images: the upper right block corresponds to strings going from the four D7-branes to their images, while the lower left block corresponds to strings with opposite orientation (in fact, they have opposite charges with respect to the diagonal U(1)). Giving a VEV to both $\phi_{\mathbf{6}_{+2}}$ and $\phi_{\mathbf{6}_{-2}}$ recombines some of the four D7-branes with some of the image D7-branes. On the other hand, $\phi_{\mathbf{1 6}}$, that is in the

\footnotetext{
${ }^{21}$ It should also have no non-chiral zero modes, but this also generically holds in our case: non-chiral zero modes living on the curve $\mathcal{C}$ where a D7 brane and a D3 intersect arise from elements of $H^{i}\left(\mathcal{C},\left.E\right|_{\mathcal{C}} \otimes\right.$ $\left.\left.F^{\vee}\right|_{\mathcal{C}} \otimes N_{\mathcal{C} \mid X}\right)$ where $\left.E\right|_{\mathcal{C}}$ and $\left.F\right|_{\mathcal{C}}$ are the bundles on the intersecting branes restricted to $\mathcal{C}$, and $N_{\mathcal{C} \mid X}$ is the normal bundle of $\mathcal{C}$ in the ambient space [92]. In our case $\mathcal{C}$ is topologically a $T^{2}$ (for both $\kappa=1$ and $\kappa=2$ ), and the bundle $\left.\left.E\right|_{\mathcal{C}} \otimes F^{\vee}\right|_{\mathcal{C}} \otimes N_{\mathcal{C} \mid X}$ is degree zero (because there are no chiral zero modes). A generic degree zero bundle on $T^{2}$ has no sections, and therefore there are no non-chiral zero modes for generic choices of flux.

${ }^{22}$ We use a different basis with respect to the usual matrix notation for the adjoint of $\mathrm{SO}(8)$ where the matrices are simply antisymmetric.
} 
adjoint of $\mathrm{U}(4)$, describes deformations and the recombinations of the $\mathrm{U}(4)$ stacks (with the analogous process in the image stack).

The D7-branes, after switching on a non-zero VEV for $\Phi$, is described by the Tachyon matrix

$$
T+\Phi,
$$

where $T$ is the tachyon describing the $\mathrm{SO}(8)$ stack. The knowledge of the tachyon matrix (with its domain and codomain) allows to derive the D-brane charges of the stack, as we will show shortly.

In presence of an orientifold projection with involution $\xi \mapsto-\xi$ for some coordinate $\xi$, the full tachyon (describing the invariant D7-brane configuration that cancels the O7-plane tadpole) must satisfy the condition [94]:

$$
T=\xi S+A
$$

where $S$ and $A$ take the following form: ${ }^{23}$

$$
S=\left(\begin{array}{cc}
M_{S} & S_{1} \\
S_{2} & M_{S}^{T}
\end{array}\right) \quad \text { and } \quad A=\left(\begin{array}{cc}
M_{A} & A_{1} \\
A_{2} & -M_{A}^{T}
\end{array}\right),
$$

where $M_{S, A}$ are generic $N \times N$ matrices, $S_{1,2}$ are symmetric $N \times N$ matrices and $A_{1,2}$ are antisymmetric $N \times N$ matrices. ${ }^{24}$

Let us come back to our setup, where we have an orientifold plane at $x_{5}=0$ and four D7-branes (plus their four images) on the same locus. Before giving VEV to $\Phi$, the tachyon of this configuration is given by (in our case $x_{5}=\xi$ ):

$$
T=\left(\begin{array}{cc}
x_{5} \mathbf{1}_{4} & 0 \\
0 & x_{5} \mathbf{1}_{4}
\end{array}\right) \text {. }
$$

We need to specify also the domain and codomain of this map. These are related to the flux on the branes $[90,94]$. In the chosen setup, where all the four D7-branes have the same flux, we have

$$
\begin{array}{cccc}
\mathcal{O}\left(-\frac{D_{5}}{2}-F+2 B\right)^{\oplus 4} & & \mathcal{O}\left(\frac{D_{5}}{2}-F+2 B\right)^{\oplus 4} \\
\mathcal{O}\left(-\frac{D_{5}}{2}+F\right)^{\oplus 4} & \rightarrow & & \\
& & \mathcal{O}\left(\frac{D_{5}}{2}+F\right)^{\oplus 4}
\end{array}
$$

where $F$ and the $B$-field are defined in (4.16) and (4.15)..$^{25}$

We now want to switch on a T-brane background, i.e. a VEV for $\Phi$ where either only $\phi_{\mathbf{6}_{+2}}$ or only $\phi_{\mathbf{6}_{-2}}$ gets a non-zero VEV. In $[95,96]$ the authors studied what are the conditions that allow a stable T-brane configuration. These are compatible with what studied in [97] with a different language.

\footnotetext{
${ }^{23}$ Here we use a different basis with respect to [94], where $S(A)$ is a symmetric (antisymmetric) matrix.

${ }^{24}$ The first $N$ lines (and columns) refer to a set of $N$ branes, while the last $N$ lines (and columns) refer to their $N$ images.

${ }^{25}$ The orientifold symmetry imposes constraints also on domain and codomain, that involve also the $B$-field.
} 
Let us say we want to give VEV only to $\phi_{6_{+2}}$. This field is a section of $\mathcal{O}\left(D_{5}-2 F+\right.$ $2 B)=\mathcal{O}\left(D_{5}-2 \mathcal{F}\right)$ (with values in the representation $\mathbf{6}_{+2}$ ). We can switch on a holomorphic VEV only when this line bundle is effective, that is for $J$ in the Kähler cone [95, 96]

$$
0 \leq \int_{D_{5}} J \wedge\left(D_{5}-2 \mathcal{F}\right)=4\left(\left(6-4 n_{b}\right) t_{b}+t_{3}+t_{4}\right)
$$

after taking the limit $t_{2} \rightarrow 0$. The r.h.s. is always positive when $n_{b} \leq 1$.

In order for this VEV to make the D-term vanish, one needs a proper sign for the FI term. ${ }^{26}$ The proper sign requirement is again given by [95-97] and is $\xi>0$ for non-zero VEV to $\phi_{\mathbf{6}_{+2}}$ (of course, the opposite sign holds for $\phi_{\mathbf{6}_{-2}}$ ). Looking at (4.19), we see that this is realised for $n_{b} \geq 1$.

We immediately see that these conditions fix the flux along $D_{b}$ to be $n_{b}=1$. As one can check, in order to find a non-zero VEV satisfying these constraints it was necessary to have a half-integral B-field along the $D_{b}$ direction. Applying the same reasoning to $\phi_{6_{-2}}$ one obtains $n_{b}=0$.

The deformation $\Phi$ does not change the D-brane charges that are given by (4.26). If $T: E_{\text {domain }} \rightarrow E_{\text {codomain }}$, the D-brane charge of the D7-stack is

$$
\Gamma_{D 7}=e^{-B}\left(\operatorname{ch}\left(E_{\text {codomain }}\right)-\operatorname{ch}\left(E_{\text {domain }}\right)\right)\left(1+\frac{c_{2}(X)}{24}\right) .
$$

In our case

$$
\begin{aligned}
\operatorname{ch}\left(E_{\text {domain }}\right) & =4\left(e^{-\frac{D_{5}}{2}-F+2 B}+e^{-\frac{D_{5}}{2}+F}\right), \\
\operatorname{ch}\left(E_{\text {codomain }}\right) & =4\left(e^{\frac{D_{5}}{2}-F+2 B}+e^{\frac{D_{5}}{2}+F}\right) .
\end{aligned}
$$

The D-brane charge of the O7-plane at $x_{5}=0$ is:

$$
\Gamma_{O 7}=-8 D_{5}+D_{5} \frac{D_{5}^{2}+c_{2}(X)}{6} .
$$

Summing the $\mathrm{D} 7$ and the $\mathrm{O} 7$ contributions, $\Gamma=\Gamma_{D 7}+\Gamma_{O 7}$, we actually see that all charges cancel except the D3-charge, that is computed to be (for both $n_{b}=1$ and $n_{b}=0$ )

$$
Q_{D 3}=-\left.\int_{X} \Gamma\right|_{6-\text { form }}=-\left(48+16 n_{2}-16 n_{2}^{2}\right) .
$$

This number should be added to the positive D3-charge of the D3-branes at the $\mathrm{dP}_{5}$ singularity which is given by 8 for the large version of model I. This leaves space for switching on 3-form fluxes necessary for stabilising dilaton and complex structure moduli.

Sp(1) D7-brane configuration. We now consider zero B-field, i.e. $B=0$. We cancel the D7-charge of the O7-plane by a stack of two branes wrapping an invariant locus $P(x)=0$ (with $P(x)$ a polynomial of degrees $(8,4,4,4)$ in all coordinates except $\left.x_{5}\right)$ in the class

\footnotetext{
${ }^{26}$ From the $4 \mathrm{D}$ point of view, the two off-diagonal blocks are related to modes with different charges with respect to the $\mathrm{U}(1)$; for a given sign of the FI term, only one charge can get VEV if the other has zero VEV.
} 
$D_{D 7}=4 D_{5}$. Moreover we consider a flux $F$ on one brane and a flux $-F$ on the second brane, that is the orientifold image of the first one. The configuration is then orientifold invariant. The corresponding tachyon matrix is

$$
T=\left(\begin{array}{cc}
P(x) & 0 \\
0 & -P(x)
\end{array}\right)
$$

with

$$
\begin{array}{cccc} 
& \mathcal{O}\left(-2 D_{5}-F\right) \\
T: & \oplus & & \mathcal{O}\left(2 D_{5}-F\right) \\
& \mathcal{O}\left(-2 D_{5}+F\right)
\end{array} \quad \rightarrow \quad \begin{gathered}
\oplus \\
\end{gathered}
$$

Since the divisor wrapped by the branes is even (hence spin), a properly quantised flux $F$ is

$$
F=m_{b} D_{b}+m_{2} D_{2}+m_{3} D_{3}+m_{4} D_{4} \quad \text { with } \quad m_{b}, m_{2}, m_{3}, m_{4} \in \mathbb{Z} .
$$

In order for the rank-2 E3-instantons to generate a non-perturbative term in the superpotential, they should have zero chiral modes at the intersection with the D7-branes. The rank-2 bundle $\mathcal{E}_{\kappa}$ supported on the E3-instantons wrapping $D_{\kappa}(\kappa=3,4)$ has the charge vector given by

$$
\Gamma_{E 3_{\kappa}}=D_{\kappa} \operatorname{ch}\left(\mathcal{E}_{\kappa}\right) \sqrt{\frac{\operatorname{Td}\left(T D_{\kappa}\right)}{\operatorname{Td}\left(N D_{\kappa}\right)}} \quad \kappa=3,4
$$

where $\operatorname{ch}(\mathcal{E})$ is the Chern character of $\mathcal{E}, \operatorname{Td}(V)$ is the Todd class of the bundle $V$ and $T D_{\kappa}$ and $N D_{\kappa}$ are respectively the tangent and the normal bundle of the surface $D_{\kappa}$. Due to the condition (4.14), one has $c_{1}\left(\mathcal{E}_{\kappa}\right)=D_{\kappa}$ and consequently

$$
\Gamma_{E 3_{\kappa}}=D_{\kappa}(2+\omega)
$$

where $\omega$ is a four-form that depends on the choice of the bundle $\mathcal{E} \cdot{ }^{27}$ From this charge vector we see that the rank-2 instantons have not chiral spectrum at the intersection with the D7-branes when the pullback of $F$ on $D_{\kappa}$ is equal to zero, i.e. when $m_{3}=m_{4}=0$. Hence the flux on the D7-branes reduces to

$$
F=m_{b} D_{b}+m_{2} D_{2} \quad \text { with } \quad m_{b}, m_{2} \in \mathbb{Z} .
$$

The flux generated FI-term is

$$
\begin{aligned}
\xi_{D 7} & =\frac{1}{4 \pi \mathcal{V}} \int_{D 7} \mathcal{F} \wedge J=\frac{1}{4 \pi \mathcal{V}} 4 D_{5} \cdot F \cdot\left(t_{b} D_{b}+t_{2} D_{2}+t_{3} D_{3}+t_{4} D_{4}\right) \\
& =\frac{1}{\pi \mathcal{V}} 8 n_{b} t_{b}-4 m_{2} t_{2} \stackrel{t_{2} \rightarrow 0}{\longrightarrow} \frac{8}{\pi \mathcal{V}} m_{b} t_{b} \simeq \frac{8}{\pi \mathcal{V}^{2 / 3}}\left(\frac{3}{2}\right)^{1 / 3} m_{b} .
\end{aligned}
$$

Let us see what is the T-brane VEV $\Phi$ that we can switch on. Now $\Phi$ is a $2 \times 2$ matrix acting on the same spaces as the tachyon, see (4.32). The upper-right element must be of the form $x_{5} Q$, where $Q$ is a holomorphic section, that happens when

$$
0 \leq \int_{D_{D 7}} J \wedge\left(D_{D 7}-2 F-D_{5}\right)=16\left(4\left(3-m_{b}\right) t_{b}+3 t_{3}+3 t_{4}\right)
$$

\footnotetext{
${ }^{27}$ If $\mathcal{E}$ is the dual of the holomorphic tangent bundle of $D_{4}$, we have $\omega=\frac{11}{12} \operatorname{ch}_{2}(\mathcal{E})$.
} 
in the limit $t_{2} \rightarrow 0$. The r.h.s. is always positive when $m_{b}<3$ (in the Kähler cone $t_{3,4}<0$ ). Switching on an upper-right element is compatible with a positive FI-term, i.e. $m_{b}>0$, see (4.37). Hence, $m_{b}$ can take the values 1,2 .

The D-brane charge is given by (4.28), where now domain and codomain are as in (4.32). Hence, $\operatorname{ch}\left(E_{\text {domain }}\right)=e^{-2 D_{5}-F}+e^{-2 D_{5}+F}$, while $\operatorname{ch}\left(E_{\text {codomain }}\right)=e^{2 D_{5}-F}+e^{2 D_{5}+F}$.

Summing the D7 and the $\mathrm{O} 7$ contributions and integrating over the $\mathrm{CY} X$, we obtain

$$
\begin{aligned}
Q_{D 3} & =-\int_{X}\left(\Gamma_{D 7}+\Gamma_{O 7}\right)_{6-\text { form }} \\
& =-\left(144+32 m_{b}^{2}-16 m_{2}^{2}\right)=\left\{\begin{array}{l}
-\left(176-16 m_{2}^{2}\right) \text { for } m_{b}=1 \\
-\left(272-16 m_{2}^{2}\right) \text { for } m_{b}=2
\end{array} .\right.
\end{aligned}
$$

We see that with this configuration we obtain a much larger (negative) D3-charge than considering the $\mathrm{SO}(8)$ stack. This is good because it allows a bigger choice in the fluxes needed to stabilise the complex structure moduli. The other difference is the multiplicity of the instanton contributing to the superpotential and that affects the exponential relation between the volume of $X$ and the volume of the divisor $D_{4}$ in the LVS minimum.

\section{Moduli stabilisation}

As a final step towards a fully fledged string compactification, we stabilise all closed string moduli in a de Sitter minimum via the LARGE Volume Scenario (LVS) [37, 98, 99]. The relevant moduli fields are $h_{-}^{1,2}$ complex structure moduli $U_{\alpha}$, the axio-dilaton $S=g_{s}^{-1}+\mathrm{i} C_{0}$ and $h_{+}^{1,1}$ Kähler moduli $T_{i}=\tau_{i}+\mathrm{i} \rho_{i}$. Here, the $\tau_{i}$ measure 4-cycle volumes of $X$ and the associated axions are given by $\rho_{i}=\int_{\mathcal{D}_{i}} C_{4}$. As seen in section 4 , for our model we have $h_{-}^{1,1}=0, h_{+}^{1,2}=7, h_{-}^{1,2}=45$ and $h_{+}^{1,1}=h^{1,1}(X)=4$. Here, we work with the volume expression in the singular limit $t_{2} \rightarrow 0$ :

$$
\mathcal{V}=d_{1} \tau_{b}^{3 / 2}-d_{3} \tau_{3}^{3 / 2}-d_{4} \tau_{4}^{3 / 2}, \quad d_{1}=d_{3}=d_{4}=\frac{1}{3 \sqrt{2}}
$$

For a globally embedded model we have to consider all the fields in the full superpotential

$$
W=W_{\text {flux }}(U, S)+W_{Q}(X, U)+W_{\mathrm{np}}(T, U, S, \varphi) .
$$

Here, $W_{\text {flux }}$ is the typical Gukov-Vafa-Witten flux superpotential [100] and $W_{Q}$ the quiver superpotential defined in (2.71). Further, we introduce a non-perturbative superpotential $W_{\text {np }}$ depending on Kähler moduli $T$ and extra D7 matter fields $\varphi$. In a similar spirit, we define the complete Kähler potential as

$$
K=K_{S}+K_{\mathrm{cs}}-2 \ln \left(\mathcal{V}+\frac{\xi}{2}\left[\frac{S+\bar{S}}{2}\right]^{3 / 2}\right)+K_{Q}(U, \bar{U}, X, \bar{X}, \mathcal{V})+K_{D 7}(S, \bar{S}, \varphi, \bar{\varphi})
$$

where

$$
K_{S}(S, \bar{S})=-\ln (S+\bar{S}), \quad K_{\mathrm{cs}}(U, \bar{U})=-\ln \left(-i \int_{X} \Omega \wedge \bar{\Omega}\right), \quad \xi=-\frac{\chi(X) \zeta(3)}{2(2 \pi)^{3}} .
$$


We take into account the $\left(\alpha^{\prime}\right)^{3}$-corrections derived in [101] which are required for the LVS [37]. In addition, the Kähler potentials $K_{Q}$ and $K_{D 7}$ for the matter fields $X_{a b}$ and $\varphi_{i}$ are $[102,103]$

$$
K_{Q}=\frac{A(U, \bar{U})}{\mathcal{V}^{2 / 3}} \operatorname{Tr}\left(X_{a b} X_{a b}^{\dagger}\right), \quad K_{D 7}=\frac{1}{S+\bar{S}} \sum_{i} \operatorname{Tr}\left(\left|\varphi_{i}\right|^{2}\right)
$$

In particular, $A(U, \bar{U})$ is an unknown function of the complex structure moduli.

For moduli stabilisation purposes, we are interested in the $\mathcal{N}=1$ scalar potential

$$
V=V_{F}+V_{D} \quad \text { with } \quad V_{F}=\mathrm{e}^{K}\left(K^{A \bar{B}} D_{A} W \overline{D_{B} W}-3|W|^{2}\right) .
$$

In the subsequent analysis, the $4 \mathrm{D}$ scalar potential can be treated as an expansion in $\mathcal{V}^{-1} \ll 1$ starting with $\mathcal{O}\left(\mathcal{V}^{-2}\right)$ at leading order. In the full minimisation of the scalar potential the F-term conditions $D_{S} W=0, D_{U} W=0$ and $D_{X} W=0$ come at the leading $\mathcal{O}\left(\mathcal{V}^{-2}\right)$ order together with $D$-terms. The minimisation with respect to the $T$ fields comes at next order $\mathcal{O}\left(\mathcal{V}^{-3}\right)$ where SUSY is broken spontaneously through non-vanishing F-terms. Due to the extended no-scale structure, additional perturbative corrections to $K$ such as from Kaluza-Klein string loops $\mathcal{O}\left(g_{s}^{2} \alpha^{\prime 2}\right)$ or from winding loops $\mathcal{O}\left(g_{s}^{2} \alpha^{4}\right)$ appear effectively at higher order in $g_{\mathrm{s}}$ and $1 / \mathcal{V}$ [104-107]. Further corrections from higher derivative $F^{4}$ terms at $\mathcal{O}\left(\alpha^{\prime 3}\right)$ are again suppressed by additional factor of $1 / \mathcal{V}$ [108]. We refer to [109] for a systematic analysis of perturbative corrections to the low-energy scalar potential of F-theory/IIB compactifications.

\subsection{Background fluxes and D-terms}

To leading order in $\mathcal{V}^{-1} \ll 1$, we find a scalar potential induced by three-form background fluxes $G_{3}$ and D-terms. At this order, it suffices to consider the tree level Kähler potential $K$ and flux induced superpotential $W_{\text {flux }}[100]$ (setting $M_{p}=1$ )

$$
K_{\text {tree }}=-\ln (S+\bar{S})-\ln \left(-i \int_{X} \Omega \wedge \bar{\Omega}\right)-2 \ln \mathcal{V} \quad W_{\text {flux }}=\int_{X} G_{3} \wedge \Omega
$$

inducing a supergravity F-term scalar potential of no-scale type

$$
V_{F}^{\text {flux }}=\mathrm{e}^{K_{\text {tree }}}\left(\left|D_{S} W_{\text {flux }}\right|^{2}+\sum_{\alpha=1}^{h_{-}^{1,2}}\left|D_{U_{\alpha}} W_{\text {flux }}\right|^{2}+\sum_{a, b}\left|D_{X_{a b}}\left(W_{Q}+W_{\text {flux }}\right)\right|^{2}\right) .
$$

The axio-dilaton and all complex structure moduli are fixed at a Minkowski minimum by solving $D_{S} W_{\text {flux }}=D_{U_{\alpha}} W_{\text {flux }}=0$ which is ensured by positive semi-definiteness of $V_{F}^{\text {flux }}{ }^{28}$ In fact, fluxes also enter the quiver superpotential $W_{Q}(2.71)$ accompanied by additional 18 free complex parameters stemming from bi-fundamental VEVs (2.50). Therefore we conclude that there are sufficiently many degrees of freedom to satisfy the quiver $F$-term conditions $D_{X_{a b}} W=0$. At this order of approximation, the minimum leaves the Kähler moduli

\footnotetext{
${ }^{28}$ At large complex structure, general expressions for flux vacua have recently been analysed in [110].
} 
directions flat and generically breaks supersymmetry because $D_{T_{i}} W_{\text {flux }}=K_{T_{i}} W_{\text {flux }} \neq 0$ whenever $G_{3}$ has a non-trivial $(0,3)$ component [111].

Further $\mathcal{O}\left(\mathcal{V}^{-2}\right)$ contributions arise from D-terms which split into a bulk and local (quiver) potential

$$
V_{D}=V_{D}^{\text {bulk }}+V_{D}^{\text {quiver }} .
$$

The former is associated with the anomalous U(1)'s living on the D7-stack wrapped around the O7-plane, whereas the latter stems from the D3-brane at the $\mathrm{dP}_{5}$ singularity. The bulk D-term potential in the convention of [35] is given by

$$
V_{D}^{\text {bulk }}=\frac{1}{2 \operatorname{Re}\left(f_{D 7}\right)}\left(\sum_{i} q_{\varphi_{i}} \frac{\left|\varphi_{i}\right|^{2}}{\operatorname{Re}(S)}-\xi_{D 7}\right)^{2},
$$

where the FI-parameters $\xi_{D 7}$ have been defined in (4.19) for the $\mathrm{SO}(8)$ and in (4.37) for the $\operatorname{Sp}(1)$ configuration. Furthermore, we defined the U(1) charges $q_{\varphi_{i}}$ of the $\varphi_{i}$ as well as the hidden sector gauge kinetic function

$$
f_{D 7}=\frac{2 T_{b}-T_{2}-T_{3}-T_{4}}{2 \pi} .
$$

We approximate

$$
\operatorname{Re}\left(f_{D 7}\right) \simeq \frac{2 \tau_{b}}{2 \pi} \simeq \frac{1}{\pi} \frac{1}{d_{1}^{\frac{2}{3}}} \mathcal{V}^{2 / 3}
$$

and consider without loss of generality a single canonically normalised charged matter field $\varphi$ so that

$$
V_{D}^{\text {bulk }}=\frac{c_{1}}{\mathcal{V}^{2 / 3}}\left(q_{\varphi}|\varphi|^{2}-\frac{c_{2}}{\mathcal{V}^{2 / 3}}\right)^{2},
$$

where the coefficients $c_{1}$ and $c_{2}$ are given by

$$
c_{1}=\frac{\pi d_{1}^{\frac{2}{3}}}{2} \quad \text { and } \quad c_{2}=\frac{1}{\sqrt{2} \pi d_{1}^{1 / 3}} \begin{cases}\left(2 n_{b}-1\right) & \mathrm{SO}(8) \\ 8 m_{b} & \mathrm{Sp}(1) .\end{cases}
$$

As for $V_{F}^{\text {flux }}$, the bulk D-term potential is positive semi-definite with a minimum at $V_{D}^{\text {bulk }}=$ 0 stabilising $\varphi$ as

$$
|\varphi|^{2}=\frac{c_{2}}{q_{\varphi} \mathcal{V}^{2 / 3}}
$$

The quiver D-term potential for the anomalous U(1) and canonically normalised matter fields $X_{a b}$ reads

$$
V_{D}^{\text {quiver }}=\frac{1}{2 \operatorname{Re}\left(f_{D 3}\right)}\left(Q^{(a b)} \operatorname{Tr}\left(X_{a b}^{\dagger} X_{a b}\right)-\xi_{D 3}\right)^{2}, \quad f_{D 3}=\frac{S}{2 \pi}, \quad \xi_{D 3} \simeq \frac{\tau_{2}}{\mathcal{V}} .
$$

As discussed in section 2.7, the cancellation of the non-abelian quiver D-terms already implies $Q_{i}^{(a b)} \operatorname{Tr}\left(X_{a b}^{\dagger} X_{a b}\right)=0$ for all U(1) charges $Q_{i}$. Thus $\xi_{D 3}=0$ for the anomalous U(1) at the minimum which puts the $\mathrm{dP}_{5}$ volume to zero, $\tau_{2}=0$. Hence, the local model is set at the singularity. 


\section{$5.2 \quad$ Non-perturbative and $\alpha^{\prime}$ effects}

Stabilisation of the remaining flat directions necessitates effects breaking the no-scale structure induced by perturbative contributions to the tree-level Kähler potential as well as non-perturbative corrections to the superpotential. In what follows, we assume that the $S$ and $U$-moduli are stabilised at their tree-level minimum which is only minorly affected by quantum corrections.

In the remainder of this section, we work with the full $\left(\alpha^{\prime}\right)^{3}$-corrected Kähler potential $K=K_{S}+K_{\mathrm{cs}}+K_{\alpha^{\prime}}$ where $[101]$

$$
K_{\alpha^{\prime}}=-2 \ln \left(\mathcal{V}+\frac{\zeta}{2}\right) \quad \text { with } \quad \zeta=-\frac{\chi(X) \zeta(3)}{2(2 \pi)^{3} g_{s}^{3 / 2}}
$$

In the model of section 4 , the E3-instantons wrapping the other two $\mathrm{dP}_{5}$ cycles introduce exponential terms in the superpotential:

$$
W=W_{0}+A_{3} e^{-a_{3} T_{3}}+A_{4} e^{-a_{4} T_{4}},
$$

where $W_{0}=\left\langle\int_{X} G_{3} \wedge \Omega\right\rangle$ denotes the VEV of the flux superpotential and $a_{3}=a_{4}=2 \pi$ for the $\mathrm{SO}(8)$ D7-brane configuration, whereas $a_{3}=a_{4}=4 \pi$ for two rank-2 instantons compatible with the $\mathrm{Sp}(1) \mathrm{D} 7$-configuration. Plugging (5.17) and (5.18) into (5.6) gives rise to the F-term scalar potential,

$$
V_{F}=V_{\alpha^{\prime}}+V_{\mathrm{np} 1}+V_{\mathrm{np} 2},
$$

where (writing $W_{0}=\left|W_{0}\right| e^{\mathrm{i} \theta_{0}}$ and $\left.A_{i}=\left|A_{i}\right| e^{\mathrm{i} \theta_{i}}\right)$ :

$$
\begin{aligned}
V_{\alpha^{\prime}}= & \frac{12 \zeta\left|W_{0}\right|^{2}}{(2 \mathcal{V}+\zeta)^{2}(4 \mathcal{V}-\zeta)} \\
V_{\mathrm{np} 1}= & \sum_{i=3}^{4} \frac{8\left|W_{0}\right|\left|A_{i}\right| e^{-a_{i} \tau_{i}} \cos \left(a_{i} \rho_{i}+\theta_{0}-\theta_{i}\right)}{(2 \mathcal{V}+\zeta)(4 \mathcal{V}-\zeta)}\left(4 a_{i} \tau_{i}+\frac{3 \zeta}{(2 \mathcal{V}+\zeta)}\right) \\
V_{\mathrm{np} 2}= & \sum_{i=3}^{4}\left\{\frac{16 a_{i}^{2} \sqrt{\tau_{i}}\left|A_{i}\right|^{2} e^{-2 a_{i} \tau_{i}}}{3 d_{i}(2 \mathcal{V}+\zeta)}+\frac{4\left|A_{i}\right|^{2} e^{-2 a_{i} \tau_{i}}}{(2 \mathcal{V}+\zeta)(4 \mathcal{V}-\zeta)}\left(8 a_{i} \tau_{i}\left(a_{i} \tau_{i}+1\right)+\frac{3 \zeta}{(2 \mathcal{V}+\zeta)}\right)\right\} \\
& +\frac{8\left|A_{3}\right|\left|A_{4}\right| e^{-a_{3} \tau_{3}-a_{4} \tau_{4}} \cos \left(a_{3} \rho_{3}-a_{4} \rho_{4}+\theta_{0}-\theta_{3}-\theta_{4}\right)}{(2 \mathcal{V}+\zeta)(4 \mathcal{V}-\zeta)} \\
& \times\left(4\left(a_{3} \tau_{3}+a_{4} \tau_{4}+2 a_{3} a_{4} \tau_{3} \tau_{4}\right)+\frac{3 \zeta}{(2 \mathcal{V}+\zeta)}\right)
\end{aligned}
$$

In the large volume limit $\mathcal{V} \gg \zeta$, the above potential can be approximated as a typical LVS scalar potential of the form

$$
V_{\mathrm{LVS}}=\sum_{i=3}^{4}\left(\frac{8 a_{i}^{2} \sqrt{\tau_{i}}\left|A_{i}\right|^{2} e^{-2 a_{i} \tau_{i}}}{3 d_{i} \mathcal{V}}+\frac{4 a_{i}\left|W_{0}\right|\left|A_{i}\right| \tau_{i} e^{-a_{i} \tau_{i}} \cos \left(a_{i} \rho_{i}+\theta_{0}-\theta_{i}\right)}{\mathcal{V}^{2}}\right)+\frac{3 \zeta\left|W_{0}\right|^{2}}{4 \mathcal{V}^{3}}
$$

The axion is fixed at

$$
\frac{a_{i}}{2 \pi} \rho_{i}=k+\frac{1}{2}+\frac{\left(\theta_{i}-\theta_{0}\right)}{2 \pi} \quad \text { with } \quad k \in \mathbb{Z} .
$$


In addition, the LVS potential (5.23) receives contributions due to soft scalar masses of the open string modes $\varphi$ which read

$$
V_{\text {soft }}=m_{\varphi}^{2}|\varphi|^{2}
$$

Without loss of generality, we restrict to a single canonically normalised visible sector matter field $X$. Generally, the soft scalar masses $m_{0}$ can be written as

$$
m_{0}^{2}=m_{3 / 2}^{2}-F^{I} F^{\bar{I}} \partial_{I} \partial_{\bar{J}} \ln \tilde{K}
$$

in terms of the gravitino mass $m_{3 / 2}$, the moduli F-terms and the Kähler metric for matter fields $\tilde{K}$. For $\varphi$, we simply have $\tilde{K}_{\varphi}=1 / \operatorname{Re}(S)$ and, since $S$ is stabilised supersymmetrically at leading order, $F^{S}=0$ which ensures that the hidden sector matter field $\varphi$ has a mass of the order of the gravitino mass

$$
m_{\varphi}^{2}=m_{3 / 2}^{2}=e^{K}|W|^{2}=\frac{e^{K_{\mathrm{cs}}}\left|W_{0}\right|^{2}}{2 \operatorname{Re}(S) \mathcal{V}^{2}}
$$

All in all, the contribution from soft scalar masses becomes

$$
V_{\mathrm{soft}}=\frac{c_{2} m_{3 / 2}^{2}}{q_{\varphi} \mathcal{V}^{2 / 3}}
$$

where we plugged in the D-term stabilisation condition (5.15) for $\varphi$.

Collecting all the formulas, the total F-term scalar potential becomes

$$
V_{\text {tot }}=\frac{e^{K_{\mathrm{cs}}}}{2 \operatorname{Re}(S)}\left(V_{\mathrm{LVS}}+\frac{\mathcal{F}_{\mathrm{up}}\left|W_{0}\right|^{2}}{\mathcal{V}^{8 / 3}}\right) \quad \text { with } \quad \mathcal{F}_{\mathrm{up}}=\frac{c_{2}}{q_{\varphi}}>0 .
$$

Notice that $\mathcal{F}_{\text {up }}>0$ can be ensured for $n_{b}, m_{b} \geq 1$ for the respective D7-brane flux configuration (assuming $q_{\varphi}>0$ ). In the limit $\epsilon_{i}=\frac{1}{4 a_{i} \tau_{i}} \ll 1$, the global minimum of (5.29) is given by

$$
\begin{aligned}
\mathcal{V} & =\frac{3 d_{i} \sqrt{\tau_{i}}\left(1-4 \epsilon_{i}\right)}{4 a_{i}\left(1-\epsilon_{i}\right)} \frac{\left|W_{0}\right|}{\left|A_{i}\right|} e^{a_{i} \tau_{i}} \simeq \frac{3 d_{i} \sqrt{\tau_{i}}}{4 a_{i}} \frac{\left|W_{0}\right|}{\left|A_{i}\right|} e^{a_{i} \tau_{i}} \\
e^{a_{3} \tau_{3}-a_{4} \tau_{4}} & =\frac{a_{3}\left|A_{3}\right| d_{4}}{a_{4}\left|A_{4}\right| d_{3}} \frac{1-\epsilon_{3}}{1-4 \epsilon_{3}} \frac{1-4 \epsilon_{4}}{1-\epsilon_{4}} \frac{\sqrt{\tau_{4}}}{\sqrt{\tau_{3}}} \simeq \frac{a_{3}\left|A_{3}\right| d_{4}}{a_{4}\left|A_{4}\right| d_{3}} \frac{\sqrt{\tau_{4}}}{\sqrt{\tau_{3}}} \\
\frac{\zeta}{2} & =\sum_{i=3}^{4} \frac{d_{i}\left(1-4 \epsilon_{i}\right)}{\left(1-\epsilon_{i}\right)^{2}} \tau_{i}^{3 / 2}-\frac{16 \mathcal{F}_{\mathrm{up}}}{27} \mathcal{V}^{1 / 3} \simeq d_{3} \tau_{3}^{3 / 2}+d_{4} \tau_{4}^{3 / 2}-\frac{16 \mathcal{F}_{\mathrm{up}}}{27} \mathcal{V}^{1 / 3} .
\end{aligned}
$$

At this minimum, we determine the vacuum energy as

$$
\left\langle V_{\text {tot }}\right\rangle \simeq \frac{e^{K_{\mathrm{cs}}}\left|W_{0}\right|^{2}}{18 \operatorname{Re}(S) \mathcal{V}^{3}}\left[\mathcal{F}_{\mathrm{up}} \mathcal{V}^{1 / 3}-\sum_{i=3}^{4} \frac{27 d_{i}}{4 a_{i}} \frac{\left(1-4 \epsilon_{i}\right)}{\left(1-\epsilon_{i}\right)^{2}} \sqrt{\tau_{i}}\right] .
$$

A Minkowski or dS vacuum is achieved by tuning the gauge and background fluxes so that

$$
\mathcal{F}_{\text {up }} \mathcal{V}^{1 / 3} \geq \sum_{i=3}^{4} \frac{27 d_{i}}{4 a_{i}} \frac{\left(1-4 \epsilon_{i}\right)}{\left(1-\epsilon_{i}\right)^{2}} \sqrt{\tau_{i}}
$$


Plugging this result with equality sign back in (5.32) we find

$$
\frac{\zeta}{2}=\sum_{i=3}^{4} \frac{d_{i}\left(1-4 \epsilon_{i}\right)\left(1-16 \epsilon_{i}\right)}{\left(1-\epsilon_{i}\right)^{2}} \tau_{i}^{3 / 2}
$$

At leading order in $\epsilon_{i} \ll 1$, we obtain

$$
\mathcal{F}_{\mathrm{up}} \mathcal{V}^{1 / 3} \gtrsim \frac{27}{4}\left(\frac{d_{3} \sqrt{\tau_{3}}}{a_{3}}+\frac{d_{4} \sqrt{\tau_{4}}}{a_{4}}\right), \quad \frac{\zeta}{2} \simeq d_{3} \tau_{3}^{3 / 2}+d_{4} \tau_{4}^{3 / 2}
$$

We conclude that the volumes of the two blow up $\mathrm{dP}_{5}$ 's at the minimum only depend on the $\alpha^{\prime}$-parameter $\zeta$ and, hence, on the Euler characteristic $\chi(X)$ and the string coupling $g_{\mathrm{s}}$.

We can solve (5.34) for $\mathcal{V}$ to find the volume at the Minkowski minimum

$$
\langle\mathcal{V}\rangle_{\text {Mink. }}=\frac{1}{\mathcal{F}_{\text {up }}^{3}}\left(\sum_{i=3}^{4} \frac{27 d_{i}}{4 a_{i}} \frac{\left(1-4 \epsilon_{i}\right)}{\left(1-\epsilon_{i}\right)^{2}} \sqrt{\tau_{i}}\right)^{3} .
$$

The required value for $\left|W_{0}\right| /\left|A_{i}\right|$ can be determined from the combination with (5.30).

Since $\epsilon_{i} \ll 1$ is a good approximation at sufficiently small $g_{s}$ (cf. figure 9 ), we may combine (5.37) with (5.30) and (5.36) to find

$$
\langle\mathcal{V}\rangle_{\text {Mink. }} \simeq \frac{1}{\mathcal{F}_{\text {up }}^{3}}\left(\sum_{i=2}^{3} \frac{27 d_{i}}{4 a_{i}} \sqrt{\tau_{i}}\right)^{3} \simeq \frac{3 d_{3} \sqrt{\tau_{3}}}{4 a_{3}} \frac{\left|W_{0}\right|}{\left|A_{3}\right|} e^{a_{3} \tau_{3}} .
$$

This can be solved for explicitly using

$$
A_{3}=A_{4}, \quad a_{3}=a_{4}, \quad d_{3}=d_{4}
$$

which enforces $\tau_{3}=\tau_{4}=\left(\zeta /\left(4 d_{3}\right)\right)^{2 / 3}$ and thus

$$
\langle\mathcal{V}\rangle_{\text {Mink. }} \simeq\left(\frac{27 d_{3}}{4 a_{3} \mathcal{F}_{\text {up }}}\right)^{3} \frac{2 \zeta}{d_{3}} \simeq \frac{3 d_{3}}{4 a_{3}}\left(\frac{\zeta}{4 d_{3}}\right)^{1 / 3} \frac{\left|W_{0}\right|}{\left|A_{3}\right|} e^{a_{3}\left[\zeta /\left(4 d_{3}\right)\right]^{2 / 3}} .
$$

Solving for $\left|W_{0}\right| /\left|A_{3}\right|$ leads to

$$
\frac{\left|W_{0}\right|}{\left|A_{3}\right|} \simeq \frac{4 a_{3}}{3 d_{3}}\left(\frac{27 d_{3}}{2 a_{3} \mathcal{F}_{\text {up }}}\right)^{3}\left(\frac{\zeta}{4 d_{3}}\right)^{2 / 3} e^{-a_{3}\left[\zeta /\left(4 d_{3}\right)\right]^{2 / 3}} .
$$

Generally, we expect $\left|W_{0}\right| /\left|A_{3}\right|$ to be exponentially suppressed at the Minkowski minimum. In fact, given that $e^{-a_{3}\left[\zeta /\left(4 d_{3}\right)\right]^{2 / 3}} \sim e^{-\frac{2.47}{g_{s}}}$, this behaviour resembles the expression for $\left|W_{0}\right|$ from flux choices proposed in [112].

A comment on the $\boldsymbol{h}^{\mathbf{1 , 1}}=\boldsymbol{N}>\mathbf{4}$ case. Let us briefly comment on scenarios with larger number of Kähler moduli. We assume we extend the model to $h^{1,1}=N$ with $N-2$ additional $\mathrm{dP}_{5}$ divisors without changing any of the other parameters. Assuming as above

$$
A_{i}=A_{j}, \quad a_{i}=a_{j}, \quad d_{i}=d_{j}, \quad \forall i, j=3, \ldots, N
$$


we would find that

$$
\frac{\zeta}{2} \simeq \sum_{i=3}^{N} d_{i} \tau_{i}^{3 / 2}=(N-2) d_{N} \tau_{N}^{3 / 2} \Rightarrow \tau_{N} \simeq\left(\frac{\zeta}{2(N-2) d_{N}}\right)^{2 / 3} .
$$

Then, the VEV for the volume at the Minkowski minimum is given by

$$
\langle\mathcal{V}\rangle_{\text {Mink. }} \simeq\left(\frac{27 d_{N}}{4 a_{N} \mathcal{F}_{\text {up }}}\right)^{3}\left(\sum_{i=3}^{N} \sqrt{\tau_{i}}\right)^{3}=\left(\frac{27 d_{N}}{4 a_{N} \mathcal{F}_{\text {up }}}\right)^{3}(N-2)^{3} \frac{\zeta}{2(N-2) d_{N}} .
$$

So at sufficiently small $g_{s}$ where $\epsilon_{N} \ll 1$, the volume at the Minkowski minimum is increased by a factor of $(N-2)^{2}$. Similarly, (5.30) implies

$$
\mathcal{V} \simeq \frac{3 d_{N}}{4 a_{N}}\left(\frac{\zeta}{(N-2) d_{N}}\right)^{1 / 3} \frac{\left|W_{0}\right|}{\left|A_{N}\right|} e^{a_{N}\left[\zeta /\left((N-2) d_{3}\right)\right]^{2 / 3}}
$$

Finally, we obtain

$$
\frac{\left|W_{0}\right|}{\left|A_{N}\right|} \simeq \frac{4 a_{N}}{3 d_{N}}\left(\frac{27 d_{N}}{4 a_{N}} \frac{(N-2)}{\mathcal{F}_{\mathrm{up}}}\right)^{3}\left(\frac{\zeta}{(N-2) d_{N}}\right)^{2 / 3} e^{-a_{N}\left[\zeta /\left((N-2) d_{N}\right)\right]^{2 / 3}} .
$$

Under the assumption that $\mathcal{F}_{\text {up }}$ remains constant and nothing else changes dramatically (tadpole, $\zeta$ etc.), there might not be as much tuning required for a large number of moduli.

Notice that in going from $h^{1,1}=3 \rightarrow N$, we effectively replace $\zeta \rightarrow \zeta /(N-2)$ and $\mathcal{F}_{\text {up }} \rightarrow \mathcal{F}_{\text {up }} /(N-2)$. The former helps with the tuning of $W_{0}$ (it appears in the exponential), whereas the latter helps increasing the volume at the Minkowski minimum. So overall this means a step in the right direction, albeit relying on a few strict assumptions. Another possibility to reduce the tuning on $W_{0}$ would be to consider constructions with gaugino condensation which would reduce the coefficient $a_{N}$ in the exponent in (5.46) to $a_{N} / P$ with $P \in \mathbb{N}$.

\subsection{Choices of underlying parameters}

We close our discussion of moduli stabilisation with presenting explicit choices of parameters stabilising all Kähler moduli in a dS or Minkowski minimum. The Euler characteristic $\chi(X)=-96$ potentially receives a $\mathcal{N}=1$ correction from O7/D7 contributions [113] that leads to an effective Euler characteristic ${ }^{29}$

$$
\chi_{\mathrm{eff}}=\chi(X)+2 \int_{X} D_{O 7}^{3}=-96+40=-56,
$$

We set $\left|A_{s}\right|=\left|A_{3}\right|=\left|A_{4}\right|$ which fixes $\tau_{s}=\tau_{3}=\tau_{4}$ upon using (5.31). Overall, we can thus tune only three parameters $g_{s},\left|W_{0}\right|,\left|A_{s}\right|$. The condition for a Minkowski minimum (5.36) fixes one combination of them which leaves us with two free parameters. For the $\mathrm{SO}(8)$ configuration, we set $n_{b}=1$ to find

$$
\mathcal{F}_{\text {up }}=\frac{1}{4 \pi}\left(\frac{1}{d_{1}}\right)^{1 / 3} \approx 0.1822
$$

\footnotetext{
${ }^{29}$ One should however keep in mind that this has only been computed for a configuration with one O7plane and one fully recombined invariant D7-brane. Here, we take the perspective that such a correction must also persist in our situation.
} 


\begin{tabular}{|c||c|c|c||c|c|c|}
\hline$g_{s}$ & $\left|W_{0}\right| /\left|A_{s}\right|$ & $\left\langle\tau_{s}\right\rangle$ & $\langle\mathcal{V}\rangle$ & $\left|W_{0}\right| /\left|A_{s}\right|$ & $\left\langle\tau_{s}\right\rangle$ & $\langle\mathcal{V}\rangle$ \\
\hline \hline 0.10 & $3.57 \cdot 10^{-6}$ & 3.23 & 115.6 & $2.88 \cdot 10^{-9}$ & 4.42 & 188.6 \\
\hline 0.05 & $2.22 \cdot 10^{-13}$ & 5.98 & 301.1 & $1.05 \cdot 10^{-19}$ & 8.35 & 503.2 \\
\hline 0.03 & $3.72 \cdot 10^{-23}$ & 9.63 & 626.4 & $8.38 \cdot 10^{-34}$ & 13.59 & 1057.4 \\
\hline 0.02 & $1.79 \cdot 10^{-35}$ & 14.21 & 1131.3 & $1.61 \cdot 10^{-51}$ & 20.15 & 1919.3 \\
\hline 0.01 & $1.21 \cdot 10^{-72}$ & 27.94 & 3145.2 & $6.85 \cdot 10^{-105}$ & 39.82 & 5363.7 \\
\hline
\end{tabular}

Table 4. Numerical analysis for SO(8)-configuration. Left: Minkowski minima for the effective

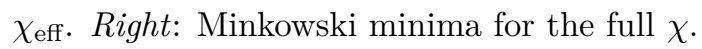

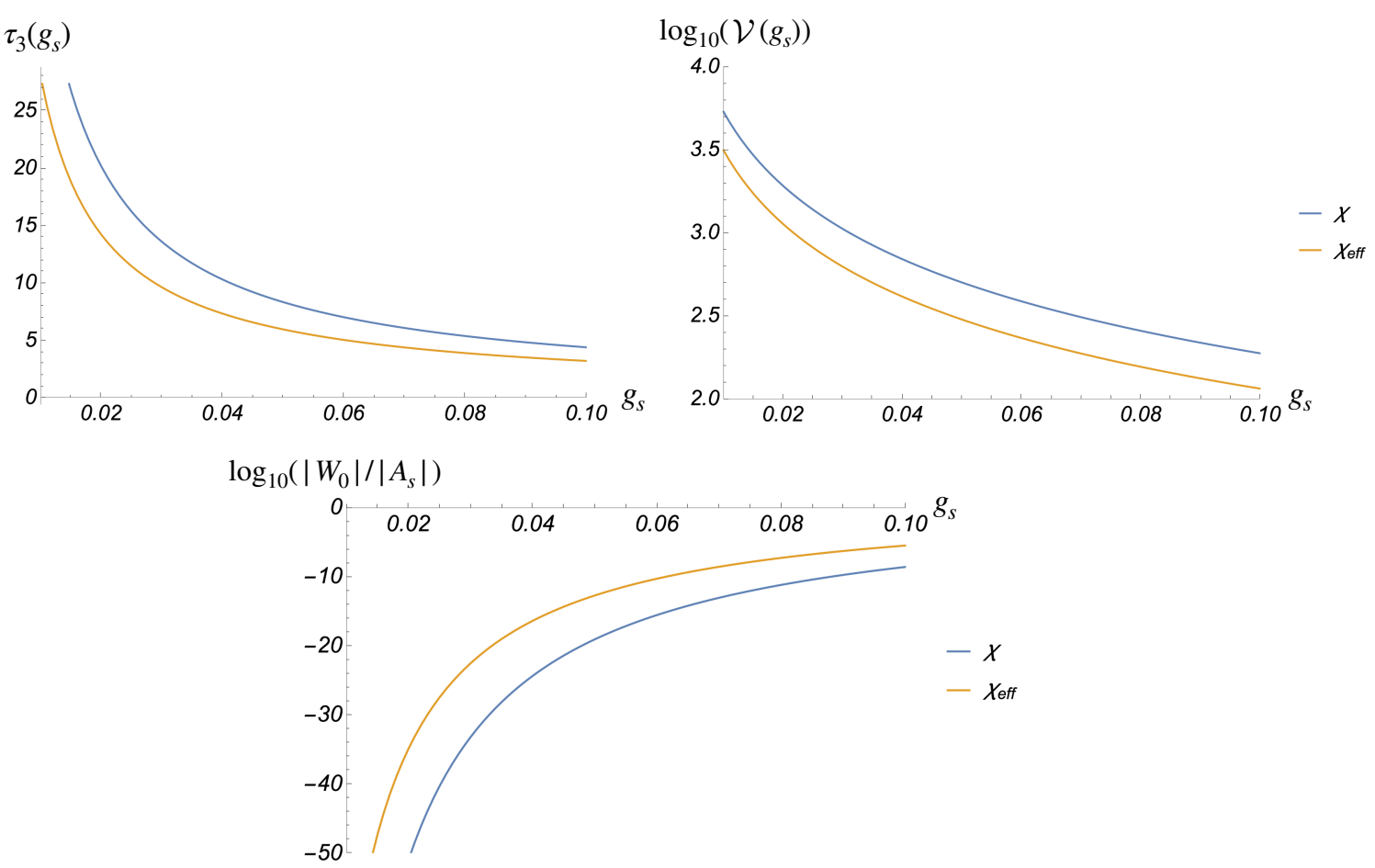

Figure 9. Values for $\tau_{s}, \mathcal{V}$ and $\left|W_{0}\right| /\left|A_{s}\right|$ for Minkowski minima for the full and effective Euler characteristic.

using that according to (4.20) the $\mathrm{U}(1)$ charge of $\varphi$ is $q_{\varphi}=2 .{ }^{30}$ We summarise our results for Minkowski minima for five values of $g_{s}$ in table 4. The general behaviour of $\tau_{s}, \mathcal{V}$ and $\left|W_{0}\right| /\left|A_{s}\right|$ as a function of $g_{s}$ is depicted in figure 9 .

The numerical results for Minkowski minima require tuning in the ratio $\left|W_{0}\right| /\left|A_{s}\right|$. While generically $\left|A_{s}\right| \sim \mathcal{O}(1)$ is expected, there are no explicit expressions available for $\left|A_{s}\right|$. In contrast, $\left|W_{0}\right|$ can be computed exactly when stabilising complex structure moduli

\footnotetext{
${ }^{30}$ For the $\mathrm{Sp}(1)$ configuration, we have $\mathcal{F}_{\text {up }}=1.4575$ which together with the two rank-2 instantons leads to even smaller VEVs for the volume at the Minkowski minimum.
} 
through fluxes. Over the past few years, progress has been made in finding flux vacua with $\left|W_{0}\right| \ll 1$ such as in [112] by studying perturbatively flat vacua so that $\left|W_{0}\right| \sim$ $\mathrm{e}^{-1 / g_{s}}$. Similarly, computer based methods from stochastic search optimisation like Genetic Algorithm have proven useful in finding solutions to $F$-term conditions with small flux superpotential [114].

\section{Phenomenological and cosmological implications}

The global $\mathrm{dP}_{5}$ model presented in the previous sections represents the first type IIB example of a realistic global model which can successfully combine: $(i)$ an explicit compact CY threefold, orientifold involution and D-brane set with tadpole cancellation; (ii) closed string moduli stabilisation in a dS minimum thanks to hidden sector T-branes [35]; (iii) a mechanism of dynamical supersymmetry breaking by the non-zero F-terms of the Kähler moduli; and (iv) exactly the MSSM gauge group and chiral matter spectrum at low energies. Moreover it features two blow-up modes which are stabilised by non-perturbative effects. Hence the CY volume form has the right structure to realise Kähler moduli inflation [39], similarly to the analysis performed in [22].

Therefore our model, after more detailed studies, could represent the first example which is both theoretically robust and fully phenomenologically viable from both the particle physics and the cosmological point of view. Notice that previous type IIB global models with dS moduli stabilisation, based on D7-branes [115], D3-branes at singularities away from the orientifold [18, 21], systems with flavour D7-branes and D3-branes at singularities far from O7-planes [19], D3-branes at orientifolded singularities [22], could reproduce points $(i),(i i)$ and $(i i i)$ but not $(i v)$ which is the major step forward of our construction.

Moduli mass spectrum. The mass spectrum of the closed string moduli is summarised in table 5. The first contributions to the $4 \mathrm{D}$ scalar potential arise at $\mathcal{O}\left(1 / \mathcal{V}^{2}\right)$ through fluxes and D-terms. The moduli stabilised at this order of approximation are the axio-dilaton $S$, the complex structure moduli $U_{\alpha}, \alpha=1, \ldots, h_{-}^{1,2}$, and the Kähler modulus $T_{2}=\tau_{2}+\mathrm{i} \rho_{2}$. The first two acquire a mass of order the gravitino mass $m_{3 / 2}$, while the mass of the $\mathrm{dP}_{5}$ volume modulus $\tau_{2}$ is of order the string scale $M_{s}$. Its axionic partner $\rho_{2}$ is instead eaten up by the anomalous $\mathrm{U}(1)$ at the singularity which develops a mass also around the string scale.

The remaining closed string moduli $T_{b}, T_{3}$ and $T_{4}$ are stabilised at order $\mathcal{O}\left(1 / \mathcal{V}^{3}\right)$ and below by $\alpha^{\prime}$ - and non-perturbative effects. The fields $\tau_{3}, \tau_{4}, \rho_{3}$ and $\rho_{4}$, associated with the blow-up modes, also receive a mass of order $m_{3 / 2}$. Even though this is of the same order as the mass of $S$ and $U_{\alpha}$, the decoupling at leading order as a result of the factorised structure of the Kähler potential (5.7) guarantees that dilaton and complex structure can be safely integrated out. Finally, the overall volume modulus $\tau_{b}$ is fixed by perturbative $\alpha^{\prime}$ corrections acquiring a mass of order $m_{3 / 2} / \sqrt{\mathcal{V}}$. Therefore, the remaining axion $\rho_{b}$ is massless at this level of approximation, but receives an exponentially small mass of order $M_{p} e^{-\mathcal{V}^{2 / 3}}$ once non-perturbative effects $\sim A_{b} e^{-2 \pi T_{b}}$ are included in the superpotential (5.18). This axionic field tends therefore to be ultra-light. 


\begin{tabular}{|c|c|c|}
\hline Field & Name & Mass \\
\hline \hline $\mathrm{dP}_{5}$ modulus & $\tau_{2}, \rho_{2}$ & $\sim M_{s}$ \\
\hline cx str moduli & $U_{\alpha}$ & $\sim m_{3 / 2}$ \\
\hline dilaton & $S$ & $\sim m_{3 / 2}$ \\
\hline blow-up cycles & $\tau_{3}, \tau_{4}$ & $\sim m_{3 / 2}$ \\
\hline blow-up axions & $\rho_{3}, \rho_{4}$ & $\sim m_{3 / 2}$ \\
\hline volume modulus & $\tau_{b}$ & $\sim m_{3 / 2} / \sqrt{\mathcal{V}}$ \\
\hline volume axion & $\rho_{b}$ & $\sim M_{p} e^{-\mathcal{V}^{2 / 3}}$ \\
\hline
\end{tabular}

Table 5. Mass spectrum of the closed string moduli where the axion $\rho_{2}$ becomes the longitudinal component of the massive anomalous $\mathrm{U}(1)$ at the $\mathrm{dP}_{5}$ singularity.

Supersymmetry breaking. The dS vacuum breaks supersymmetry dynamically due to non-vanishing F-terms

$$
\frac{F^{T_{b}}}{\tau_{b}} \sim \frac{F^{T_{3}}}{\tau_{3}} \sim \frac{F^{T_{4}}}{\tau_{4}} \sim m_{3 / 2}, \quad F^{S} \sim F^{U_{\alpha}} \sim \frac{m_{3 / 2}}{\mathcal{V}},
$$

while $F^{T_{2}}=0$ (up to volume suppressed subleading corrections) for the $\mathrm{dP}_{5}$ modulus. Given that $\tau_{b} \gg \tau_{3} \simeq \tau_{4}$, the largest $\mathrm{F}$-term is $F^{T_{b}}$, signalling that the Goldstino eaten up by the gravitino is the $T_{b}$-modulino. Gravitational interactions mediate supersymmetry breaking to the visible sector at the $\mathrm{dP}_{5}$ singularity. At first sight, given that the local Kähler modulus $T_{2}$ has a vanishing F-term, the resulting soft terms are expected to be suppressed with respect to the gravitino mass, as typical of sequestered scenarios with D3-branes at singularities [116, 117]. However, threshold corrections to the gauge kinetic function should induce a redefinition of the local $\mathrm{dP}_{5}$ modulus of the form $[118,119]$ :

$$
\tau_{2} \quad \rightarrow \quad \tau_{2}^{\text {new }}=\tau_{2}-\alpha \ln \mathcal{V},
$$

where $\alpha$ can be expressed in terms of the 1-loop $\beta$-function coefficient of the local gauge theory. Notice that this redefinition should occur for orientifolded singularities, but not for orbifolded ones. The presence of flavour D7-branes is also expected to break sequestering inducing logarithmic corrections similar to (6.2). The authors of [38] noticed that the redefinition (6.2) induces non-zero F-terms for the local $\mathrm{dP}_{5}$ modulus

$$
F^{T_{2}}=0 \quad \rightarrow \quad F^{T_{2}} \sim \alpha m_{3 / 2} .
$$

This effect breaks sequestering and all soft masses turn out to be of order the gravitino mass, $M_{\text {soft }} \sim m_{3 / 2} \sim\left|W_{0}\right| M_{p} / \mathcal{V}$. The choice of the underlying parameters corresponding to the first line of table 4 would give rise to intermediate scale soft terms, 
$M_{\text {soft }} \sim m_{3 / 2} \sim 10^{10} \mathrm{GeV}$, which can be compatible with the observed value of the Higgs mass if $\tan \beta \sim 1$. Notice, in addition, that intermediate scale supersymmetry could also be motivated by the fact that the Higgs quartic coupling in the SM vanishes exactly around $10^{10} \mathrm{GeV}$ which is the energy scale where new physics should arise to guarantee the stability of the Higgs potential. On the other hand, the second line would yield TeV-scale superpartners, $M_{\text {soft }} \sim m_{3 / 2} \sim 1 \mathrm{TeV}$ which would provide a standard solution to the hierarchy problem due to low energy supersymmetry. All the other parameter choices do not seem to be phenomenologically viable since they would give rise to soft terms below LHC scales. ${ }^{31}$ Notice that more examples with phenomenologically viable soft terms and gravitino mass could be obtained by focusing on CY threefolds with more blow-up modes, since this would reduce the tuning in $\left|W_{0}\right|$ and increase $m_{3 / 2}$, as explained in section 5.2.

Cosmology. Realising inflation in scenarios with a $\mathrm{TeV}$-scale gravitino mass is rather hard due to the well-known difficulty to combine inflation with low scale supersymmetry [120]. Moreover, in this case the volume mode would suffer from the cosmological moduli problem, unless its mass is raised above $50 \mathrm{TeV}$ which would however increase also the gravitino mass around $m_{3 / 2} \sim 1000 \mathrm{TeV}$.

We therefore focus on the case with $m_{3 / 2} \sim 10^{10} \mathrm{GeV}$ corresponding to the first line of table 4. This value of the gravitino mass is exactly in the right ballpark to reproduce the observed amplitude of the density perturbations in inflationary models where inflation is driven by one blow-up mode, say $\tau_{3}$, which is slow-rolling towards the minimum of its potential, while the overall volume is kept approximately constant by the other blowup mode, $\tau_{4}$, which is kept at its minimum, i.e. $\mathcal{V} \sim\left|W_{0}\right| e^{a_{4} \tau_{4}}$. The post-inflationary evolution of this kind of inflation models has been already analysed in several papers and can lead to non-standard thermal histories. Preheating effects have been studied in $[121,122]$ while perturbative reheating has been analysed in [40, 41]. A crucial modulus whose dynamics controls the post-inflationary evolution is $\tau_{b}$. During inflation this field gets slightly displaced from its minimum due to the inflationary energy density [123]. When the Hubble scale becomes of order of its mass, the volume mode starts oscillating and gives rise very quickly to an early epoch of matter domination. When $\tau_{b}$ decays it dilutes everything that has been produced before. This dilution mechanism can be very useful to have viable super-heavy dark matter scenarios [42] and Affleck-Dine baryogenesis [43] that would otherwise lead to an overproduction of either WIMP dark matter or matterantimatter asymmetry. Moreover, the decay of the volume mode tends to produce axionic dark radiation [44-46] which can be within observational constraints and can represent an interesting experimental signature of these constructions.

Comments on dS vacua. The $\mathrm{dS}$ vacua obtained in section 5.2 rely on a full stabilisation of all Kähler moduli in detail. We did not perform an explicit fixing of the axio-dilaton and the complex structure moduli even if we checked that the D3 tadpole cancellation condition leaves enough freedom to turn on appropriate 3 -form background fluxes which should

\footnotetext{
${ }^{31}$ Unless the prefactor of the non-perturbative effects $\left|A_{s}\right|$ can be tuned to very large values, which we consider however a very contrived situation.
} 
lift these directions at semi-classical level. We therefore argue that the stabilisation of these moduli should be under control. In [21] we exploited symmetries of the complex structure moduli space to reduce the effective number of these moduli to just a few. Similar techniques can be combined with the ones of $[112,124,125]$ to perform a full stabilisation of the dilaton and the $U$-moduli with an exponentially small flux superpotential $\left|W_{0}\right|$. Notice that the need to tune $\left|W_{0}\right|$ is model-dependent since it depends on microscopic quantities like CY intersection numbers and gauge flux quanta. In fact, in [22] we obtained similar dS vacua without the need to tune the flux superpotential. Moreover, as already stressed above, cases with more blow-up modes would reduce the tuning in $\left|W_{0}\right|$. Moreover we have shown that open string moduli can be fixed via D- and F-flatness conditions, even if a complete fixing of all these modes has still to be achieved. However we expect this to be possible via a combination of gauge and bulk fluxes, together with supersymmetry breaking effects.

Let us also mention that we managed to obtain dS minima at values of the volume of order $\mathcal{V} \sim 10^{2}-10^{3}$ which are not extremely large, but still large enough to keep a numerical, even if not parametric, control over quantum corrections. This result confirms the expectation that dS minima cannot appear at arbitrarily large volume in agreement with current swampland considerations [126]. This is because the barrier becomes increasingly small when the volume is increased, and the dS minima eventually disappear. Larger values of the volume are expected for more generic compactifications with the volume expected to increase with the square of $h^{1,1}$ as argued in section 5.2.

It is worth stressing that uplifting with T-branes is a feature that arises very naturally in type IIB string compactifications since it relies on generic features of these constructions: (i) the presence of hidden sector D7-branes which is typically forced by D7 tadpole cancellation; (ii) non-zero worldvolume fluxes which are in general required by Freed-Witten anomaly cancellation [32]; ( $i$ ii ) the need to turn on 3-form bulk fluxes for dilaton and complex structure moduli stabilisation. As explained in [35], points $(i)$ and $(i i)$ naturally induce a T-brane background, or equivalently charged matter fields fixed in terms of moduli-dependent FI terms, while point ( $i i i)$ gives rise to the positive uplifting term, corresponding to non-zero F-terms of these matter fields.

We finally stress that our global constructions provide fully explicit setups of type IIB flux compactifications where the visible sector is exactly the MSSM and the moduli can be stabilised in a dS vacuum. While achieving full control is always challenging, there is a coherent picture of viable string vacua emerging from these investigations. We strongly believe that further exploring these phenomenologically preferred string scenarios opens up new avenues to scrutinising physical implications of string compactifications.

\section{Conclusions}

Quiver gauge theories from fractional branes constitute quintessential realisations of local models with viable particle phenomenology in string compactifications. Despite that, their embedding into compact CY backgrounds remains largely unexplored. Here, the challenge is building a fully trustable and consistent global model including brane setups satisfying tadpole cancellation conditions combined with moduli stabilisation and dS uplifting. In 
recent years, some of the authors of this paper made progress in this direction by providing global embeddings of oriented [18-21] and unoriented [22] quiver gauge theories. In the case of the former, two identical del Pezzo divisors are exchanged under the orientifold involution. In the latter scenario, there is only a single del Pezzo divisor transversely invariant under the orientifold action. D-term stabilisation forces the shrinking of the divisor volume to zero size yielding a CY singularity.

The visible sector is realised on the worldvolume of D3-branes sitting at the tip of this singularity and consists of realistic extensions of the Standard Model such as trinification, Pati-Salam or SU(5) models. Additional rigid divisors in the CY threefold host nonperturbative effects which are imperative to stabilise closed string moduli together with $\alpha^{\prime}$ corrections at exponentially large volume [37]. The cancellation of Freed-Witten anomalies may induce a non-trivial flux background on the hidden sector D7-branes. In this way, a T-brane background is naturally generated leading to Minkowski or slightly de Sitter vacua [35]. In the models with orientifolded fractional branes, soft masses turn out to be of order the gravitino mass [38] which can be either at intermediate or at LHC scales. In the former case, these models can also provide a viable description of cosmic inflation as in [22].

This paper concerned the first construction of the Minimal Quiver Standard Model (MQSM) $[25,26]$ from D3-branes at del Pezzo singularities in a fully fledged type IIB CY threefold flux compactification. Specifically, we employed ideas first proposed in [23] to construct supersymmetric versions of the MQSM from a single orientifolded D3-brane at a $\mathrm{dP}_{5}$ singularity. We primarily focussed on a particular setup which led to the Minimal Supersymmetric Left-Right Symmetric Model with an additional vector like pair of Higgs doublets as an intermediate quiver gauge theory. This extension of the Standard Model addresses several open questions such as the origin of parity violation or the strong CP problem making it phenomenologically highly attractive, see for example [127] and references therein. Critically, the local model stems from a single D3-brane without the need to introduce flavour D7-branes, although the global embedding contained D7-branes passing through the singularity. While they did not introduce any additional chiral states in the quiver gauge theory, this could represent another source of desequestering of the visible sector realisation.

Subsequently, we searched for compact CY geometries suited for accommodating the local model. Among the many constraints on such a global embedding, the diagonality constraints imposed on the collapsing $\mathrm{dP}_{5}$ divisor turned out to be more restrictive than initially anticipated. This condition on the triple intersection numbers was necessary to ensure that the shrinking of the $\mathrm{dP}_{5}$ volume does not force any other divisors to shrink in which case the visible sector would look rather different.

In a first attempt, we studied the KS database [31] of 4-dimensional reflexive polytopes where CY threefolds are represented as fine, regular, star triangulations. We randomly scanned over a range of Hodge numbers $h^{1,1} \leq 40$ utilising the software package CYTools [87]. None of the $\approx 350.000$ distinct geometries exhibited diagonal $\mathrm{dP}_{n}$ divisors with $1 \leq n \leq 5$. In fact, these results were exhaustive for $h^{1,1} \leq 5$ possibly hinting at a deeper underlying reason. This observation led us to formulate a conjecture about the absence of such divisor structures in the KS database in general. Clearly, a much more 
thorough analysis is required to quantify the validity of this conjecture. We stress however that we found $\approx 3000$ distinct geometries involving diagonal $\mathrm{dP}_{n}$ divisors with $n \geq 6$ whose quiver gauge theories can be related to the one of $\mathrm{dP}_{5}$ via Higgsing, see e.g. [63]. In this sense, the KS database contains CY threefolds implicitly related to the types of constructions studied throughout this paper. We hope to come back to these models in the near future.

To proceed, we utilised the basic fact that $\mathrm{dP}_{5}$ surfaces can be constructed as biquadrics in $\mathbb{P}^{4}$. Thus, the strategy to constructing $\mathrm{CY}$ threefolds with (diagonal) $\mathrm{dP}_{5}$ divisors became looking for complete intersections of two equations with five-dimensional toric spaces. The bi-quadric is recovered upon setting one coordinate to zero and properly gauge fixing all but one of the $\mathbb{C}^{*}$ actions. In this way, we obtained several $\mathrm{CY}$ threefolds for $2 \leq h^{1,1} \leq 4$ with diagonal $\mathrm{dP}_{5}$ divisors together with additional del Pezzo divisors supporting Euclidean D3-instantons.

In the remainder of the paper, we focussed on one particular geometry and presented a concrete embedding of the local model. We studied a particular involution that required only a single O7-plane on a large 4-cycle. Here, we showed using the techniques of [128] that in a local neighbourhood around the singularity the global orientifold involution can be related to the well-understood line orientifold of complex cones over $\mathrm{dP}_{5}$ at a special locus in complex structure moduli space [83, 128, 129].

The D7-tadpole induced by the O7-plane was cancelled by adding four D7-branes plus their images on top of the O7-plane. The resulting hidden sector $\mathrm{SO}(8)$ gauge group was broken to U(4) by worldvolume fluxes which were required by Freed-Witten anomaly cancellation [32]. The induced FI-term forced the adjoint scalar on the D7 worldvolume to gain a VEV generating a T-brane background thereby ensuring bulk D-term cancellation. Ultimately, this led to a positive contribution to the $4 \mathrm{~d}$ scalar potential from scalar soft masses and therewith to a well-controlled de Sitter uplift [35]. The additional $\mathrm{dP}_{5}$ divisors were wrapped by rank-1 ED3-instantons which were critical for closed string moduli stabilisation. We used the standard LVS [37] to find SUSY-breaking minima by balancing non-perturbative effects against perturbative $\alpha^{\prime}$ corrections. As usual, supersymmetry was broken spontaneously in the hidden sector by non-vanishing F-terms for bulk Kähler moduli mediating SUSY breaking to the visible sector via gravitational couplings. Our model has also all the right features to realise Kähler moduli inflation [39] and to give rise to a viable post-inflationary evolution with interesting observational implications, as described in section 6 .

In summary, this paper is a first step towards having fully-fledged string constructions with geometric moduli stabilised and the MSSM or its Left-Right extension. We have followed the three steps mentioned in the introduction and in some sense it is a culmination of accumulated progress over the years on both the model building and moduli stabilisation. We have illustrated that combining the two into global string constructions with concrete CY compactifications is highly non-trivial but achievable. We also illustrated the richness of these constructions that somehow complements and generalises the F-theory constructions to the cases where it is not the fibre but the base that is singular. This is a very large and promising class of models that has not been much explored so far. 
In the future, we aim at considering a systematic approach to more generic models with gaugino condensation and a higher number of Kähler moduli that would naturally provide further realistic properties and could open new avenues towards inflation. This may also help the numerics to allow larger volumes with not so small values of $W_{0}$, as argued in section 5.2. Furthermore, our realisation of the left-right symmetric model could provide a golden opportunity to study all the interesting properties of the left-right symmetric models within concrete string models, providing a UV completion for these interesting phenomenological models (see [130-132] for recent discussions). Having explicit low-energy string models, there are plenty of flavour questions that may need to be studied before claiming to have a fully realistic model.

On the local side, it remains an unresolved issue to construct explicit orientifold actions on exceptional collections of fractional branes. While the construction of exceptional collections is systemically possible using the techniques of [67], the resulting collections are generically incompatible with the standard "large volume" orientifold action. This has been partially explored in [133] for the case of $\mathbb{C}^{3} / \mathbb{Z}_{3}$. However, the situation is far from clear for del Pezzo singularities.

Similarly, on the global embedding side, there continue to be open challenges like the inclusion of $\mathrm{U}(1)$ instantons. In addition, recent software developments such as cohomCalg [88, 89] and CYTools [87] should allow for a systematic classification of viable global models with D-branes at singularities. While most model building strategies thus far have been based on a case by case study, this would constitute a huge leap towards treating large classes of string compactifications simultaneously.

\section{Acknowledgments}

We thank Christoph Mayrhofer for initial collaboration on this project and valuable input. We gratefully acknowledge discussions with Alex Cole, Arthur Hebecker, Sven Krippendorf, Jakob Moritz and Gary Shiu. We would like to thank the SISSA/ICTP HPC Cluster for allowing the access, and in particular Benvenuto Bazzo, Ivan Girotto and Johannes Grassberger at ICTP for their crucial technical help in scanning geometries. The work of FQ has been partially supported by STFC consolidated grants ST/P000681/1, ST/T000694/1. AS acknowledges support by the German Academic Scholarship Foundation, by DAMTP through an STFC studentship as well as by the Cambridge Trust through a Helen Stone Scholarship. AS also thanks ICTP, Trieste, for hospitality during the initial stages of this work. I.G.E. is supported in part by STFC through grant ST/T000708/1. M.C. and R.V. acknowledge support by INFN Iniziativa Specifica ST\&FI.

\section{A Analysing the CY threefolds from the KS database}

\section{A.1 Diagonal dP divisors in CY hypersurfaces in toric ambient spaces}

In this section, we discuss the challenges for the global embedding of our local $\mathrm{dP}_{5}$ model using the $\mathrm{CY}$ threefolds arising from the four-dimensional reflexive polytopes listed in the KS database [31]. As mentioned in the first requirement of the list, we need to search for 
CY threefolds $\left(X_{3}\right)$ which could have at least one (diagonal) $\mathrm{dP}_{5}$ divisor. For that purpose, we utilized the topological data of $\mathrm{CY}$ threefolds from the polytope triangulations presented in [86], which we refer as AGHJN-database. In the search of $\mathrm{dP}_{5}$ divisor, we focus only on looking at the topology of the so-called "coordinate divisors" $D_{i}$ which are defined through setting $x_{i}=0$. However this is sufficient for capturing the del Pezzo surfaces as the non-coordinate divisors, which could arise from considering the combinations of various coordinate divisors, would not be rigid. With this underlying strategy, our plan for looking at the suitable del Pezzo divisors is twofold: first we scan for the divisors $D_{s}$ which satisfy the topological conditions (3.1). Using this information, we subsequently impose the 'diagonality' condition (3.2) on each of the del Pezzo divisor $D_{s}$. For all the $\mathrm{dP}_{5}$ toric divisors which we have obtained for the $\mathrm{CY}$ threefolds in the KS database using the triangulation of the AGHJN-database [86], we find that the diagonality condition (3.2) could never be satisfied for the $\mathrm{dP}_{5}$ divisors. In fact, most of the times we find that the volume of $\mathrm{dP}_{5}$ four-cycle takes the following form,

$$
\tau_{\mathrm{dP}_{5}}=\left(\sum_{i} a_{i} t^{i}\right)\left(\sum_{j} b_{j} t^{j}\right) \quad \text { for some } i \neq j .
$$

To illustrate the volume form for a $\mathrm{dP}_{5}$ divisor to take of the form as given in eq. (A.1), one can consider the explicit $\mathrm{CY}$ threefolds with a $\mathrm{dP}_{5}$ divisor presented in $[84,134]$. The main problem with the volume of the form (A.1) is that one cannot shrink such $\mathrm{dP}_{5}$ divisors to a point-like singularity via squeezing along a single direction. This is what we call a 'nondiagonal' del Pezzo. In this case, squeezing along a single direction results in a line-like singularity, and one has to squeeze the $\mathrm{dP}_{5}$ divisor from two directions to get a point-like singularity.

\section{A.2 Scanning results for (diagonal) del Pezzo divisors}

All the various scans which we will present in this article will correspond to the so-called 'favourable' triangulations (Triang*) and 'favourable' geometries (Geom*) [86]. In fact, in non-favourable CY threefolds, the number of toric divisors in the basis is less than $h^{1,1}(C Y)$, and subsequently there is always at least one coordinate divisor which is nonsmooth, and often turns out to be a disjoint union of two del Pezzo surfaces. We exclude such spaces from our scan; several of them can be described as complete intersections in higher dimensional toric spaces. Moreover, we look for the presence of del Pezzo surfaces of any degree (not just $\mathrm{dP}_{5}$ ). ${ }^{32}$ The results of our search are collected in tables 6 and $7 .{ }^{33}$ To present the number of CY threefolds which could support the standard LVS [37], we have created separate column "LVS" in table 7 which correspond to the CY threefolds having at least one 'diagonal' del Pezzo divisor.

\footnotetext{
${ }^{32}$ This will be useful in future work for embedding generic local $\mathrm{dP}_{n}$ models.

${ }^{33}$ Let us mention that the sum of the individual counting of spaces having a particular type of $\mathrm{dP}_{n}$ is reflected to be quite large, e.g. as can be seen from table 6, this sum is even larger than the total number of CY threefolds for $h^{1,1}=4$. This is because of the fact that there can be multiple (types of) del Pezzo divisors within the same CY threefold.
} 


\begin{tabular}{|c||c|c||c|c|c|c|c|c|c|c|c|}
\hline$h^{1,1}$ & Poly* $^{*}$ & Geom* & $\mathrm{dP}_{0}$ & $\begin{array}{c}\mathrm{dP}_{1} \text { or } \\
\mathbb{F}_{0}\end{array}$ & $\mathrm{dP}_{2}$ & $\mathrm{dP}_{3}$ & $\mathrm{dP}_{4}$ & $\mathrm{dP}_{5}$ & $\mathrm{dP}_{6}$ & $\mathrm{dP}_{7}$ & $\mathrm{dP}_{8}$ \\
\hline 1 & 5 & 5 & 0 & 0 & 0 & 0 & 0 & 0 & 0 & 0 & 0 \\
2 & 36 & 39 & 9 & 4 & 0 & 0 & 0 & 0 & 2 & 4 & 5 \\
3 & 243 & 305 & 55 & 88 & 4 & 4 & 2 & 9 & 20 & 62 & 64 \\
4 & 1185 & 2000 & 304 & 767 & 146 & 135 & 52 & 175 & 213 & 566 & 506 \\
5 & 4897 & 13494 & 2107 & 6518 & 1960 & 2094 & 880 & 2005 & 2011 & 4358 & 3837 \\
\hline
\end{tabular}

Table 6. Number of CY geometries with a particular type of del Pezzo divisor.

\begin{tabular}{|c||c|c||c|c|c|c|c|c||c|}
\hline$h^{1,1}$ & Poly $^{*}$ & $\begin{array}{c}\text { Geom } \\
\left(n_{\mathrm{CY}}\right)\end{array}$ & $\mathrm{ddP}_{0}$ & $\begin{array}{c}\mathrm{ddP}_{1} \text { or } \\
d \mathbb{F}_{0}\end{array}$ & $\begin{array}{c}\mathrm{ddP}_{n} \\
2 \leq n \leq 5\end{array}$ & $\mathrm{ddP}_{6}$ & $\mathrm{ddP}_{7}$ & $\mathrm{ddP}_{8}$ & $\begin{array}{c}n_{\text {LVS }} \\
\left(\mathrm{ddP}_{n} \geq 1\right)\end{array}$ \\
\hline 1 & 5 & 5 & 0 & 0 & 0 & 0 & 0 & 0 & 0 \\
2 & 36 & 39 & 9 & 2 & 0 & 2 & 4 & 5 & 22 \\
3 & 243 & 305 & 55 & 16 & 0 & 16 & 37 & 34 & 132 \\
4 & 1185 & 2000 & 304 & 140 & 0 & 97 & 210 & 126 & 750 \\
5 & 4897 & 13494 & 2107 & 901 & 0 & 486 & 731 & 374 & 4104 \\
\hline
\end{tabular}

Table 7. Number of CY geometries with a 'diagonal' del Pezzo divisor suitable for LVS.

Note that both the scanning approaches regarding the divisor topologies, either by looking at the Intersection Tensor satisfying the eq. (3.1) or by considering the Hodge Diamond using the cohomCalg [88, 89], do not distinguish between the divisors $\mathbb{F}_{0}=\mathbb{P}^{1} \times \mathbb{P}^{1}$ and $\mathrm{dP}_{1}$ as both the surfaces are described by a set of Hodge numbers $\left\{h^{0,0}=1, h^{0,1}=\right.$ $\left.0, h^{0,2}=0, h^{1,1}=2\right\}$. In order to make this distinction, we have looked at the circumstances when a surface can be a $\mathrm{dP}_{1}$ and when it cannot. This can be checked by considering the following triple-intersections for a given divisor $D_{s}$ with the above mentioned Hodge numbers, which could either be a $\mathbb{F}_{0}$ or a $\mathrm{dP}_{1}$ surface,

$$
\int_{C Y} D_{s}^{2} D_{i}=m, \quad \int_{C Y} D_{s} D_{i}^{2}=n \quad s \neq i .
$$

Now

- if at least one of the intersection numbers $m, n \in\{2 k+1: k \in \mathbb{Z}\}$ for some divisor $D_{i}$, then the corresponding divisor $D_{s}$ is a $\mathrm{dP}_{1}$. This simple condition is sufficient however not necessary, and it turns out to be quite strong to capture the $\mathrm{dP}_{1}$ surfaces.

- for the cases where all the $m \in\{2 k: k \in \mathbb{Z}\}$, the situation can get a bit subtle to make a conclusion as it might be possible that all divisors in the CY restrict to the same (even) homology class in the surface. However one can determine the cases when the divisor cannot be a $\mathrm{dP}_{1}$. This can be checked by failing to find a solution of the following conditions for a given even number $m=2 k$ :

$$
2 k=p^{2}-q^{2} \quad \text { for some } p, q \in \mathbb{Z}, \quad k \in \mathbb{Z}^{*}
$$




\begin{tabular}{|c||c|c||c|c|c|c|c|c|c|c|c|c|}
\hline$h^{1,1}$ & Poly $^{*}$ & Geom $^{*}$ & $\mathrm{dP}_{0}$ & $\mathbb{F}_{0}$ & $\mathrm{dP}_{1}$ & $\mathrm{dP}_{2}$ & $\mathrm{dP}_{3}$ & $\mathrm{dP}_{4}$ & $\mathrm{dP}_{5}$ & $\mathrm{dP}_{6}$ & $\mathrm{dP}_{7}$ & $\mathrm{dP}_{8}$ \\
\hline 1 & 5 & 5 & 0 & 0 & 0 & 0 & 0 & 0 & 0 & 0 & 0 & 0 \\
2 & 36 & 39 & 9 & 2 & 2 & 0 & 0 & 0 & 0 & 2 & 4 & 5 \\
3 & 243 & 305 & 55 & 20 & 68 & 4 & 4 & 2 & 9 & 20 & 62 & 64 \\
4 & 1185 & 2000 & 304 & 166 & 601 & 146 & 135 & 52 & 175 & 213 & 566 & 506 \\
5 & 4897 & 13494 & 2107 & 1203 & 5315 & 1960 & 2094 & 880 & 2005 & 2011 & 4358 & 3837 \\
\hline
\end{tabular}

Table 8. Number of CY geometries with a particular type of del Pezzo divisor.

\begin{tabular}{|c||c|c||c|c|c|c|c|c||c|}
\hline$h^{1,1}$ & Poly $^{*}$ & $\begin{array}{c}\text { Geom* }^{*} \\
\left(n_{\mathrm{CY}}\right)\end{array}$ & $\mathrm{ddP}$ & $d \mathbb{F}_{0}$ & $\begin{array}{c}\mathrm{ddP}_{n} \\
1 \leq n \leq 5\end{array}$ & $\mathrm{ddP}_{6}$ & $\mathrm{ddP}_{7}$ & $\mathrm{ddP}_{8}$ & $\begin{array}{c}n_{\text {LVS }} \\
\left(\mathrm{ddP}_{n} \geq 1\right)\end{array}$ \\
\hline 1 & 5 & 5 & 0 & 0 & 0 & 0 & 0 & 0 & 0 \\
2 & 36 & 39 & 9 & 2 & 0 & 2 & 4 & 5 & 22 \\
3 & 243 & 305 & 55 & 16 & 0 & 16 & 37 & 34 & 132 \\
4 & 1185 & 2000 & 304 & 140 & 0 & 97 & 210 & 126 & 750 \\
5 & 4897 & 13494 & 2107 & 901 & 0 & 486 & 731 & 374 & 4104 \\
\hline
\end{tabular}

Table 9. Number of CY geometries with a 'diagonal' del Pezzo divisor suitable for LVS.

Or

$$
\exists k_{1}, k_{2} \in 2 \mathbb{Z}^{*}: \quad \frac{k_{2}}{k_{1}}=\left(\frac{p}{q}\right)^{2} \quad \text { for some } p, q \in \mathbb{Q} \text {. }
$$

In our case, it turns out that there are no solutions to each of these conditions and hence ensuring that the corresponding divisor cannot be a $\mathrm{dP}_{1}$.

- assuming that the divisors with $\left\{h^{0,0}=1, h^{0,1}=0, h^{0,2}=0, h^{1,1}=2\right\}$ appearing in our scan are either $\mathrm{dP}_{1}$ or $\mathbb{F}_{0}=\mathbb{P}^{1} \times \mathbb{P}^{1}$, we consider the number of $\mathbb{F}_{0}$ divisors as those which are guaranteed to be not a $\mathrm{dP}_{1}$ surface.

Let us also note here that we have extended our notation to denote a diagonal $\mathbb{F}_{0}=\mathbb{P}^{1} \times \mathbb{P}^{1}$ as $d \mathbb{F}_{0}$ which satisfy the condition given in eq. (3.2).

In course of making this study we have analysed all the divisor topologies for more than 60,000 CY threefolds arising from the triangulations of the four-dimensional polytopes collected in the KS database, see tables 8 and 9 . In this process, we have encountered around $2000 \mathrm{dP}_{5}$ divisors while considering the distinct $\mathrm{CY}$ geometries, and around 9000 $\mathrm{dP}_{5}$ divisors while considering the $\mathrm{CY}$ triangulations, and interestingly none of these $\mathrm{dP}_{5}$ divisors satisfy the diagonality criteria mentioned in eq. (3.2). The generic observations made in this analysis made us formulate the conjecture stated in section 3.2. 


\section{B CICY threefolds with diagonal $\mathrm{dP}_{5}$ divisors}

\section{B.1 $\mathrm{dP}_{5}$ embedded in a CICY with $h^{1,1}=2$}

The toric data for a complete intersection $\mathrm{CY}$ threefold which realizes the diagonal $\mathrm{dP}_{5}$ divisors is given as under,

\begin{tabular}{|c|c||ccccccc|}
\hline$H Y_{1}$ & $H Y_{2}$ & $x_{1}$ & $x_{2}$ & $x_{3}$ & $x_{4}$ & $x_{5}$ & $x_{6}$ & $x_{7}$ \\
\hline 3 & 3 & 1 & 1 & 1 & 1 & 1 & 1 & 0 \\
1 & 1 & 0 & 0 & 0 & 0 & 0 & 1 & 1 \\
\hline & & $\mathrm{SD} 1$ & $\mathrm{SD} 1$ & $\mathrm{SD} 1$ & $\mathrm{SD} 1$ & $\mathrm{SD} 1$ & $\mathrm{SD} 2$ & $\mathrm{dP}_{5}$ \\
\hline
\end{tabular}

with the SR ideal being given as $\left\{x_{1} x_{2} x_{3} x_{4} x_{5}, x_{6} x_{7}\right\}$. This CY threefold has the Hodge numbers $\left(h^{2,1}, h^{1,1}\right)=(66,2)$ and subsequently the Euler number $\chi=-128$. A detailed divisor analysis using cohomCalg $[88,89]$ shows that the divisor $D_{7}$ is a indeed a del Pezzo $\mathrm{dP}_{5}$ while the divisors $\left\{D_{1}, \ldots, D_{6}\right\}$ constitute two 'special deformation' divisors with Hodge diamond:

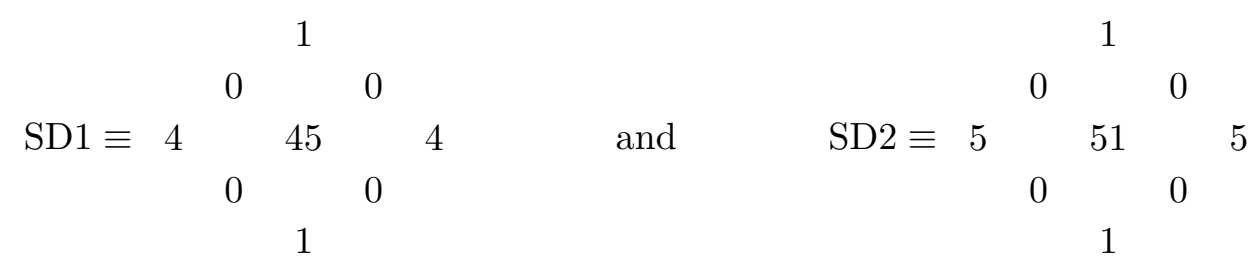

Here following the nomenclature proposed in [135] we refer the divisors with $h^{2,0}(D) \geq 2$ as "special deformation" (SD) divisors. These are special in the sense that the simplest divisor which can be deformed has $h^{2,0}(D)=1$ e.g. the well known $K 3$ surfaces. The intersection form in the basis of smooth divisors $\left\{D_{6}, D_{7}\right\}$ can be written as:

$$
I_{3}=9 D_{6}^{3}+4 D_{7}^{3} .
$$

Writing the Kähler form in the above basis of divisors as $J=t_{6} D_{6}+t_{7} D_{7}$ and using the intersection polynomial (B.1), the CY overall volume takes the form:

$$
\mathcal{V}=\frac{3 t_{6}^{3}}{2}+\frac{2 t_{7}^{3}}{3}=\frac{\sqrt{2}}{9} \tau_{6}^{3 / 2}-\frac{1}{3 \sqrt{2}} \tau_{7}^{3 / 2},
$$

where the divisor volumes are given as $\tau_{6}=\frac{9 t_{6}^{2}}{2}$ and $\tau_{7}=2 t_{7}^{2}$. Unlike the $\mathrm{dP}_{5}$ divisors embedded into CY hypersurfaces, this divisor is indeed diagonal. In other words, now we can shrink it to a point-like singularity along a single direction, namely by taking $t_{7} \rightarrow 0$. Unlike the previous conclusion drawn for the whole set of examples in the KS list, this example serves as the proof for the existence of a CICY threefold with a 'diagonal' $\mathrm{dP}_{5}$ divisor.

The Kähler cone conditions are given as below,

$$
t_{6}+t_{7}>0, \quad t_{7}<0,
$$

which show that shrinking the volume of the $d P_{5}$ divisor corresponds to approaching the boundary of the Kähler cone. 


\section{B.2 CICY examples with $h^{1,1}=3$}

Example A. Now we present a CICY threefold which has a diagonal $\mathrm{dP}_{5}$ divisor along with another diagonal $\mathrm{dP}_{n}$ divisor, in order to support our local $\mathrm{dP}_{5}$ model within a LVS framework. The toric data for such a CICY threefold is given as under,

\begin{tabular}{|c|c||cccccccc|}
\hline$H Y_{1}$ & $H Y_{2}$ & $x_{1}$ & $x_{2}$ & $x_{3}$ & $x_{4}$ & $x_{5}$ & $x_{6}$ & $x_{7}$ & $x_{8}$ \\
\hline 3 & 3 & 1 & 1 & 1 & 1 & 1 & 1 & 0 & 0 \\
2 & 0 & 0 & 0 & 0 & 0 & 0 & 1 & 1 & 0 \\
1 & 1 & 0 & 0 & 0 & 0 & 1 & 0 & 0 & 1 \\
\hline & & SD1 & SD1 & SD1 & SD1 & SD2 & SD3 & $\mathrm{dP}_{6}$ & $\mathrm{dP}_{5}$ \\
\hline
\end{tabular}

with the SR ideal being given as $\left\{x_{1} x_{2} x_{3} x_{4}, x_{5} x_{8}, x_{6} x_{7}\right\}$. This CY threefold has the Hodge numbers $\left(h^{2,1}, h^{1,1}\right)=(55,3)$ and subsequently the Euler number $\chi=-104$. A detailed divisor analysis using cohomCalg $[88,89]$ shows that the divisor $D_{8}$ is a indeed a del Pezzo $\mathrm{dP}_{5}$ while the divisor $D_{7}$ is a $\mathrm{dP}_{6}$. Further, the divisors $\left\{D_{1}, \ldots, D_{6}\right\}$ constitute three 'special deformation' type divisors with Hodge diamond:

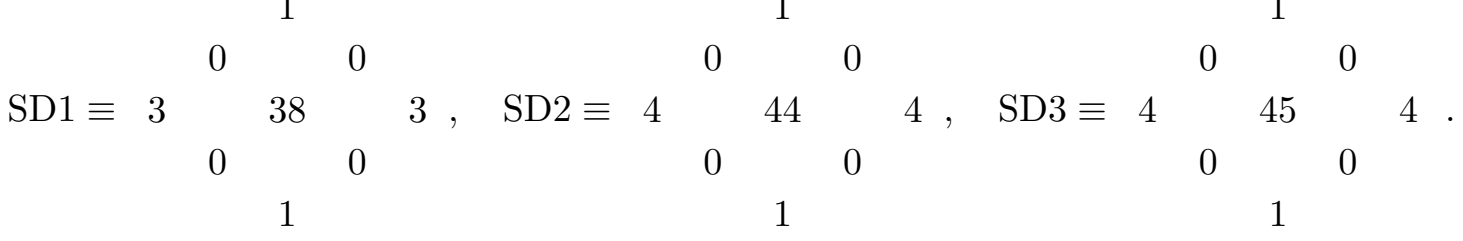

The intersection form in the basis of smooth divisors $\left\{D_{6}, D_{7}, D_{8}\right\}$ can be written as:

$$
I_{3}=5 D_{6}^{3}+3 D_{7}^{3}+4 D_{6}^{2} D_{8}-4 D_{6} D_{8}^{2}+4 D_{8}^{3} .
$$

Writing the Kähler form in the above basis of divisors as $J=t_{6} D_{6}+t_{7} D_{7}+t_{8} D_{8}$ and using the intersection polynomial (B.4), the CY overall volume takes the form:

$$
\mathcal{V}=\frac{5}{6} t_{6}^{3}+\frac{1}{2} t_{7}^{3}+2 t_{6}^{2} t_{8}-2 t_{6} t_{8}^{2}+\frac{2}{3} t_{8}^{3}=\frac{\sqrt{2}}{9}\left(\tau_{6}+\tau_{8}\right)^{3 / 2}-\frac{1}{3} \sqrt{\frac{2}{3}} \tau_{7}^{3 / 2}-\frac{1}{3 \sqrt{2}} \tau_{8}^{3 / 2},
$$

where in the second step we have used the following expressions for the divisor volumes $\tau_{6}=\frac{5 t_{6}^{2}}{2}+4 t_{6} t_{8}-2 t_{8}^{2}, \tau_{7}=\frac{3 t_{7}^{2}}{2}$ and $\tau_{8}=2\left(t_{6}-t_{8}\right)^{2}$. This volume form suggests that working in the different basis of smooth divisors $\left\{D_{x}, D_{7}, D_{8}\right\}$, the intersection polynomial reduces into the following form,

$$
I_{3}=9 D_{x}^{3}+3 D_{7}^{3}+4 D_{8}^{3},
$$

which subsequently gives the following strong swiss-cheese volume form,

$$
\mathcal{V}=\frac{\sqrt{2}}{9} \tau_{x}^{3 / 2}-\frac{1}{3} \sqrt{\frac{2}{3}} \tau_{7}^{3 / 2}-\frac{1}{3 \sqrt{2}} \tau_{8}^{3 / 2}
$$

where $\tau_{x}=\frac{9 t_{x}^{2}}{2}, \tau_{7}=\frac{3 t_{7}^{2}}{2}$ and $\tau_{8}=2 t_{8}^{2}$. Thus we ensure that both the $\mathrm{dP}_{5}$ as well as $\mathrm{dP}_{6}$ divisors are diagonal. In other words, now we can shrink it to a point-like singularity along a single direction, namely by $t_{8} \rightarrow 0$. 
Example B. Now we refine our construction a bit more by presenting a CICY threefold which has two diagonal $\mathrm{dP}_{5}$ divisors for $h^{1,1}(X)=3$. The toric data is given by,

\begin{tabular}{|c|c||cccccccc|}
\hline$H Y_{1}$ & $H Y_{2}$ & $x_{1}$ & $x_{2}$ & $x_{3}$ & $x_{4}$ & $x_{5}$ & $x_{6}$ & $x_{7}$ & $x_{8}$ \\
\hline 4 & 4 & 1 & 0 & 0 & 2 & 2 & 1 & 1 & 1 \\
2 & 2 & 0 & 1 & 0 & 1 & 1 & 0 & 0 & 1 \\
2 & 2 & 0 & 0 & 1 & 1 & 1 & 0 & 1 & 0 \\
\hline & & $\mathrm{SD} 1$ & $\mathrm{dP}_{5}$ & $\mathrm{dP}_{5}$ & $\mathrm{SD} 2$ & $\mathrm{SD} 2$ & $\mathrm{SD} 1$ & $\mathrm{SD} 3$ & $\mathrm{SD} 3$ \\
\hline
\end{tabular}

with the SR ideal being given as

$$
S R=\left\{x_{2} x_{3}, x_{2} x_{8}, x_{1} x_{6} x_{8}, x_{3} x_{4} x_{5} x_{7}, x_{1} x_{4} x_{5} x_{6} x_{7}\right\} .
$$

This CY threefold has the Hodge numbers $\left(h^{2,1}, h^{1,1}\right)=(59,3)$ and subsequently the Euler number $\chi=-112$. In fact these GLSM charges results in three triangulations such that the corresponding $\mathrm{CICY}$ has two diagonal $\mathrm{dP}_{5}$ divisors. Other two triangulations corresponds to the following SR ideas:

$$
\begin{aligned}
& S R_{1}=\left\{x_{2} x_{3}, x_{2} x_{8}, x_{3} x_{7}, x_{1} x_{4} x_{5} x_{6} x_{7}, x_{1} x_{4} x_{5} x_{6} x_{8}\right\} \\
& S R_{2}=\left\{x_{2} x_{3}, x_{3} x_{7}, x_{1} x_{6} x_{7}, x_{2} x_{4} x_{5} x_{8}, x_{1} x_{4} x_{5} x_{6} x_{8}\right\} .
\end{aligned}
$$

A detailed divisor analysis using cohomCalg $[88,89]$ shows that the two divisors $D_{2}$ and $D_{3}$ are del Pezzo $\mathrm{dP}_{5}$ surfaces while the remaining divisors constitute three types of what we call 'special deformation' divisors with the following Hodge diamonds:

$$
\begin{aligned}
& 1
\end{aligned}
$$

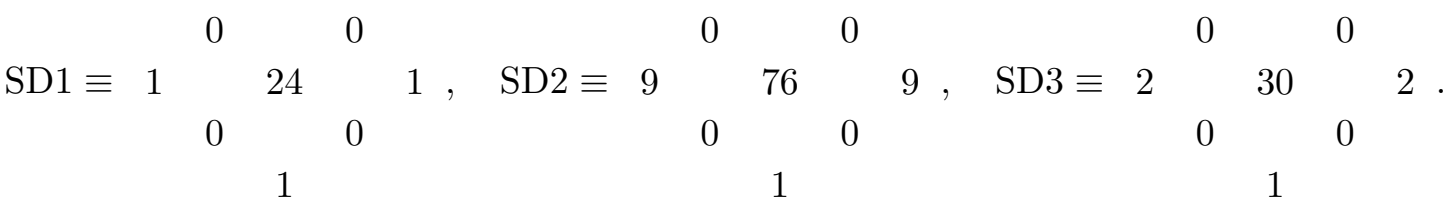

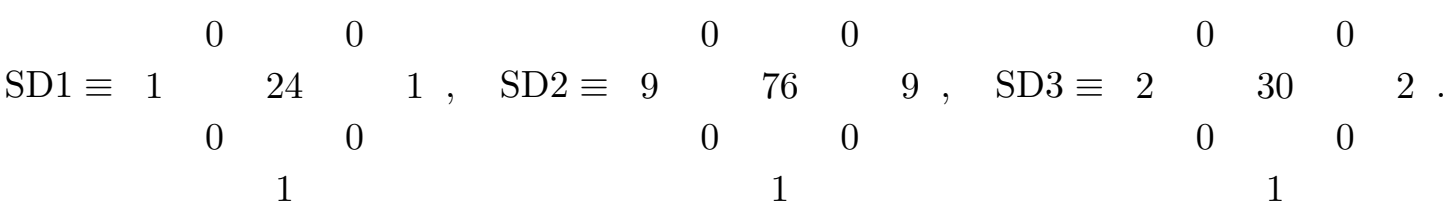

$$
\begin{aligned}
& 1
\end{aligned}
$$

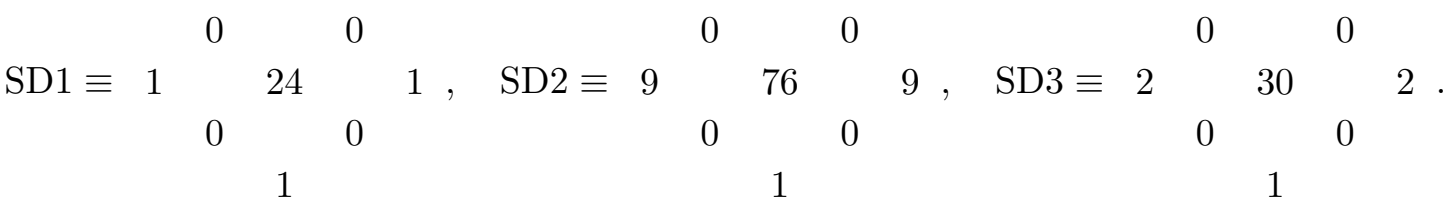

$$
\begin{aligned}
& 1
\end{aligned}
$$

In the basis of smooth divisors $\left\{D_{b}, D_{2}, D_{3}\right\}$, where is defined as $D_{b}=D_{1}+D_{2}+D_{3}$, the intersection form can be written as:

$$
I_{3}=4 D_{b}^{3}+4 D_{2}^{3}+4 D_{3}^{3},
$$

which subsequently gives the following strong swiss-cheese volume form,

$$
\mathcal{V}=\frac{1}{3 \sqrt{2}}\left(\tau_{b}^{3 / 2}-\tau_{2}^{3 / 2}-\tau_{3}^{3 / 2}\right)
$$

where the four-cycle volumes are given in terms of the two-cycle volumes as: $\tau_{b}=2 t_{b}^{2}, \tau_{2}=$ $2 t_{2}^{2}$ and $\tau_{3}=2 t_{3}^{2}$. Thus we ensure that both of the $\mathrm{dP}_{5}$ divisors are diagonal. So we can equivalently shrink any of the $\mathrm{dP}_{5}$ to a point-like singularity by squeezing along a single direction, for example say via taking $t_{3} \rightarrow 0$. 
Example C. Now we present a CICY threefold example which has one diagonal $\mathrm{dP}_{5}$ divisor along with a diagonal $\mathrm{dP}_{8}$ divisor for $h^{1,1}(X)=3$. Even though this model does not work for our construction, it might become useful in the future. The toric data for such a CICY threefold is given as under,

\begin{tabular}{|c|c||cccccccc|}
\hline$H Y_{1}$ & $H Y_{2}$ & $x_{1}$ & $x_{2}$ & $x_{3}$ & $x_{4}$ & $x_{5}$ & $x_{6}$ & $x_{7}$ & $x_{8}$ \\
\hline 4 & 6 & 1 & 0 & 0 & 3 & 2 & 2 & 1 & 1 \\
2 & 4 & 0 & 1 & 0 & 2 & 1 & 1 & 0 & 1 \\
2 & 0 & 0 & 0 & 1 & 0 & 0 & 0 & 1 & 0 \\
\hline & & $\mathrm{NdP}_{12}$ & $\mathrm{dP}_{5}$ & $\mathrm{dP}_{8}$ & $\mathrm{SD} 1$ & $\mathrm{SD} 2$ & $\mathrm{SD} 2$ & $\mathrm{SD} 3$ & $\mathrm{SD} 4$ \\
\hline
\end{tabular}

with the SR ideal being given as

$$
S R=\left\{x_{3} x_{7}, x_{2} x_{3} x_{8}, x_{2} x_{4} x_{8}, x_{1} x_{5} x_{6} x_{7}, x_{1} x_{4} x_{5} x_{6}\right\} .
$$

This CY threefold has the Hodge numbers $\left(h^{2,1}, h^{1,1}\right)=(43,3)$ and subsequently the Euler number $\chi=-80$. A detailed divisor analysis using cohomCalg $[88,89]$ shows that the two divisors $D_{2}$ and $D_{3}$ are del Pezzo $\mathrm{dP}_{5}$ and $\mathrm{dP}_{8}$ surfaces respectively while the remaining divisors constitute four types of what we call 'special deformation' (SD) divisors with the following Hodge diamonds:

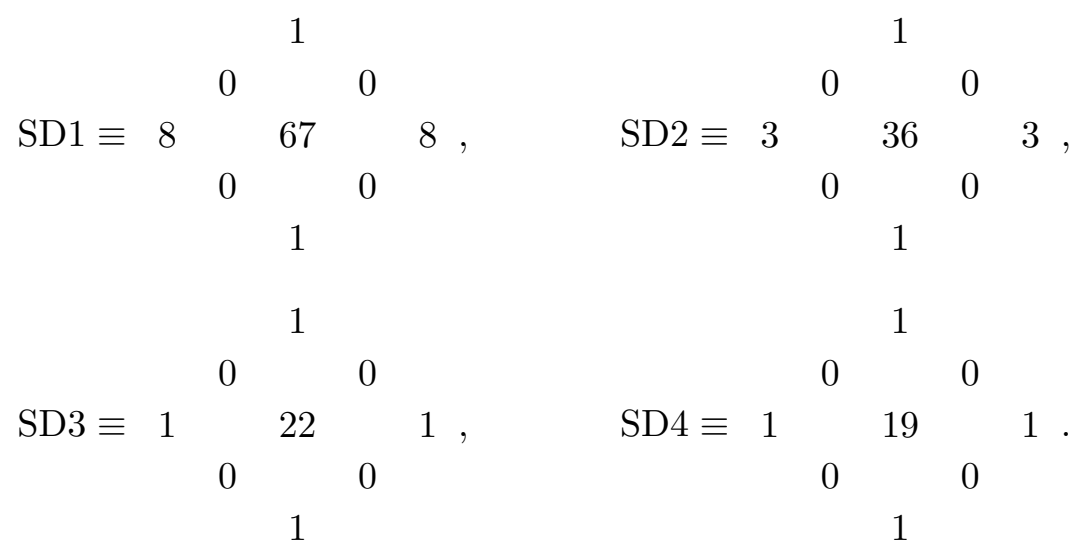

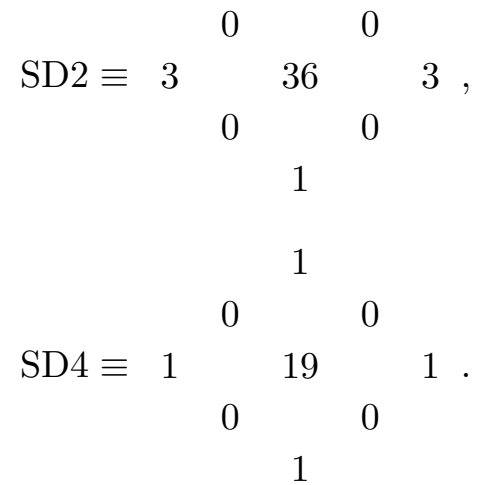

In the basis of smooth divisors $\left\{D_{b}, D_{2}, D_{3}\right\}$, where is defined as $D_{b}=D_{1}+D_{2}+D_{3}$, the intersection form can be written as:

$$
I_{3}=2 D_{b}^{3}+4 D_{2}^{3}+D_{3}^{3},
$$

which subsequently gives the following strong swiss-cheese volume form,

$$
\mathcal{V}=\frac{1}{3} \tau_{b}^{3 / 2}-\frac{1}{3 \sqrt{2}} \tau_{2}^{3 / 2}-\frac{\sqrt{2}}{3} \tau_{3}^{3 / 2}
$$

where the four-cycle volumes are given in terms of the two-cycle volumes as: $\tau_{b}=t_{b}^{2}, \tau_{2}=$ $2 t_{2}^{2}$ and $\tau_{3}=\frac{t_{3}^{2}}{2}$. Thus we ensure that both of the del Pezzo divisors, namely $\mathrm{dP}_{5}$ as well as 
$\mathrm{dP}_{8}$, are diagonal. So we can shrink the $\mathrm{dP}_{5}$ to a point-like singularity by squeezing along a single direction via taking $t_{2} \rightarrow 0$, leading to the following effective volume form:

$$
\mathcal{V}=\frac{1}{3} \tau_{b}^{3 / 2}-\frac{\sqrt{2}}{3} \tau_{3}^{3 / 2}
$$

The second Chern-class is given as:

$$
c_{2}(X)=16 D_{b}^{2}+D_{2}^{2}+10 D_{3}^{2} .
$$

\section{B.3 A CICY example with $h^{1,1}=4$}

Now we take our construction one step ahead by supporting one of the LVS models of inflation, namely the Kähler moduli inflation, in addition to have a global LVS embedding of our local chiral $\mathrm{dP}_{5}$ model. For that here present a CICY threefold which has a diagonal $\mathrm{dP}_{5}$ divisor and two other diagonal $\mathrm{dP}_{n}$ divisor. The toric data for such a CICY threefold is given as under,

\begin{tabular}{|c|c||ccccccccc|}
\hline$H Y_{1}$ & $H Y_{2}$ & $x_{1}$ & $x_{2}$ & $x_{3}$ & $x_{4}$ & $x_{5}$ & $x_{6}$ & $x_{7}$ & $x_{8}$ & $x_{9}$ \\
\hline 3 & 3 & 1 & 1 & 1 & 1 & 1 & 1 & 0 & 0 & 0 \\
2 & 0 & 0 & 0 & 0 & 0 & 0 & 1 & 1 & 0 & 0 \\
0 & 2 & 0 & 0 & 0 & 0 & 1 & 0 & 0 & 1 & 0 \\
1 & 1 & 0 & 0 & 0 & 1 & 0 & 0 & 0 & 0 & 1 \\
\hline & & $\mathrm{SD} 1$ & $\mathrm{SD} 1$ & $\mathrm{SD} 1$ & $\mathrm{SD} 2$ & $\mathrm{SD} 2$ & $\mathrm{SD} 2$ & $\mathrm{dP}_{6}$ & $\mathrm{dP}_{6}$ & $\mathrm{dP}_{5}$ \\
\hline
\end{tabular}

with the SR ideal being given as

$$
S R=\left\{x_{4} x_{9}, x_{6} x_{7}, x_{7} x_{8}, x_{7} x_{9}, x_{5} x_{8}, x_{8} x_{9}, x_{1} x_{2} x_{3} x_{4} x_{5}, x_{1} x_{2} x_{3} x_{4} x_{6}, x_{1} x_{2} x_{3} x_{5} x_{6}\right\} .
$$

This CY threefold has the Hodge numbers $\left(h^{2,1}, h^{1,1}\right)=(44,4)$ and subsequently the Euler number $\chi=-80$. A detailed divisor analysis using cohomCalg [88, 89] shows that the divisor $D_{8}$ is a indeed a del Pezzo $\mathrm{dP}_{5}$ while the divisor $D_{6}$ and $D_{7}$ divisors are both a $\mathrm{dP}_{6}$ surface. Further, the divisors $\left\{D_{1}, \ldots, D_{5}\right\}$ constitute two 'special deformation' type divisors with Hodge diamond:

$$
\begin{aligned}
& 1 \quad 1
\end{aligned}
$$

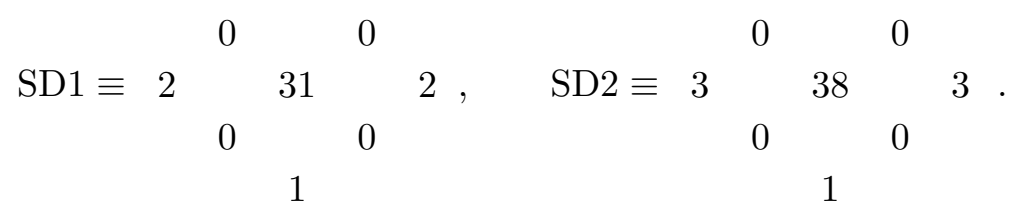

The intersection form in the basis of smooth divisors $\left\{D_{6}, D_{7}, D_{8}, D_{9}\right\}$ can be written as:

$$
I_{3}=2 D_{6}^{3}+3 D_{7}^{3}+9 D_{6}^{2} D_{8}-9 D_{6} D_{8}^{2}+3 D_{8}^{3}+4 D_{6}^{2} D_{9}-4 D_{6} D_{9}^{2}+4 D_{9}^{3} .
$$

Writing the Kähler form in the above basis of divisors as $J=t_{6} D_{6}+t_{7} D_{7}+t_{8} D_{8}$ and using the intersection polynomial (B.13), the $\mathrm{CY}$ overall volume takes the form:

$$
\begin{aligned}
\mathcal{V} & =\frac{t_{6}^{3}}{3}+\frac{t_{7}^{3}}{2}+\frac{3 t_{6}^{2} t_{8}}{2}-\frac{3 t_{6} t_{8}^{2}}{2}+\frac{t_{8}^{3}}{2}+2 t_{6}^{2} t_{9}-2 t_{6} t_{8}^{2}+\frac{2 t_{9}^{3}}{3} \\
& =\frac{\sqrt{2}}{9}\left(\tau_{6}+\tau_{8}+\tau_{9}\right)^{3 / 2}-\frac{1}{3} \sqrt{\frac{2}{3}} \tau_{7}^{3 / 2}-\frac{1}{3} \sqrt{\frac{2}{3}} \tau_{8}^{3 / 2}-\frac{1}{3 \sqrt{2}} \tau_{9}^{3 / 2},
\end{aligned}
$$


where in the second step we have used the following expressions for the divisor volumes $\tau_{6}=t_{6}^{2}+3 t_{6} t_{8}-\frac{3 t_{8}^{2}}{2}+4 t_{6} t_{9}-2 t_{9}^{2}, \tau_{7}=\frac{3 t_{7}^{2}}{2}, \tau_{8}=\frac{3\left(t_{6}-t_{8}\right)^{2}}{2}$ and $\tau_{8}=2\left(t_{6}-t_{9}\right)^{2}$. This volume form suggests that working in the different basis of smooth divisors $\left\{D_{x}, D_{7}, D_{8}, D_{9}\right\}$, the intersection polynomial reduces into the following form,

$$
I_{3}=9 D_{x}^{3}+3 D_{7}^{3}+3 D_{8}^{3}+4 D_{9}^{3},
$$

which subsequently gives the following strong swiss-cheese volume form,

$$
\mathcal{V}=\frac{\sqrt{2}}{9} \tau_{x}^{3 / 2}-\frac{1}{3} \sqrt{\frac{2}{3}} \tau_{7}^{3 / 2}-\frac{1}{3} \sqrt{\frac{2}{3}} \tau_{8}^{3 / 2}-\frac{1}{3 \sqrt{2}} \tau_{9}^{3 / 2}
$$

where $\tau_{x}=\frac{9 t_{x}^{2}}{2}, \tau_{7}=\frac{3 t_{7}^{2}}{2}, \tau_{8}=\frac{3 t_{8}^{2}}{2}$ and $\tau_{9}=2 t_{9}^{2}$. Thus we ensure that both the $\mathrm{dP}_{6}$, as well as $\mathrm{dP}_{5}$ divisors are diagonal. So we can shrink the $\mathrm{dP}_{5}$ to a point-like singularity along a single direction, namely by taking $t_{9} \rightarrow 0$.

\section{Additional material on $\mathrm{dP}_{5}$ quivers}

\section{C.1 D-terms for $\mathrm{SU}(N)$ and $\operatorname{USp}(2 N)$ gauge groups}

For the non-abelian part of the gauge group there is no FI-term. Hence the D-term reduces to

$$
D_{a} D^{a}=\kappa^{a b} D_{a} D_{b}
$$

with $D_{a}$ given by

$$
D_{a}=\sum_{i}\left\langle\varphi_{i}\left|T_{a}\right| \varphi_{i}\right\rangle=\operatorname{Tr} T_{a} \sum_{i}\left|\varphi_{i}\right\rangle\left\langle\varphi_{i}\right|=\operatorname{Tr} T_{a} H
$$

Here we introduced the hermitian matrix $H$ as an abbreviation for $\sum_{i}\left|\varphi_{i}\right\rangle\left\langle\varphi_{i}\right|$ and the $T_{a}$ 's are the generators of the non-abelian gauge group in the representation of the $\left|\varphi_{i}\right\rangle$ 's.

We denote by $t_{\alpha}$ the generators of hermitian $N \times N$-matrices, whereupon we chose a basis such that $t_{0}=\mathbb{1}, \operatorname{Tr} t_{\alpha}=0$ for $\alpha>0$ and $\operatorname{Tr} t_{\alpha} t_{\beta}=0$ for $\alpha \neq \beta$. We use this basis to expand $H$ as $H=h^{\beta} t_{\beta}$ with $h^{\beta} \in \mathbb{R}$. Furthermore, the generators of $\mathrm{SU}(N)$ are given by $t_{\alpha}$ with $\alpha>0 .{ }^{34}$ Hence we obtain for $D_{\alpha}$ :

$$
D_{\alpha}=\operatorname{Tr} t_{\alpha} h^{\beta} t_{\beta}=h^{\beta} \operatorname{Tr} t_{\alpha} t_{\beta} \propto h_{\alpha} \quad \text { with } \alpha>0 .
$$

Since $\kappa^{a b}$ is definite for simple Lie groups, we need either $H \propto \mathbb{1}$ or $H=0$ for a vanishing D-term.

\footnotetext{
${ }^{34}$ Note that this is true for the fundamental representation. In the case that there are further states transforming under this gauge group, but in a different representation, the trace in (C.2) is obviously a sum of traces over the respective representations. This means in particular, if we have states in the fundamental representation, its conjugate representation and the adjoint representation then (C.2) will look like $\operatorname{Tr} t_{a} H_{1}-\operatorname{Tr} t_{a} H_{2}+\sum_{i} \operatorname{Tr} t_{a}\left[\phi_{i}, \phi_{i}^{\dagger}\right]$, where $H_{1}$ and $H_{2}$ encode the states in the fundamental and anti-fundamental representation, respectively, and $\phi_{i}$ are the fields in the adjoint representation.
} 
For $\operatorname{USp}(2 N)$ gauge groups the situation is a bit less restrictive, as we explain in the following. The $2 N \times 2 N$-matrices $t_{a}$ generating $\operatorname{USp}(2 N)$ must be hermitian and in addition of the form

$$
t_{a}=\left(\begin{array}{cc}
A_{a} & B_{a}^{\dagger} \\
B_{a} & -A_{a}^{T}
\end{array}\right)
$$

with $\left(A=A^{\dagger}\right.$ and) $B=B^{T}$ in order to be symplectic too. To simplify notation, we also subdivide the matrix $H$ into $N \times N$-matrices:

$$
H=\left(\begin{array}{cc}
O & P \\
P^{\dagger} & Q^{T}
\end{array}\right)
$$

with $O=O^{\dagger}$ and $Q=Q^{\dagger}$. Plugging this into (C.2) we find

$$
D_{a}=\operatorname{Tr} A_{a} O+B_{a}^{\dagger} P^{\dagger}+\operatorname{Tr} B_{a} P-A_{a}^{T} Q^{T}=\operatorname{Tr} A_{a}(O-Q)+2 \Re\left(\operatorname{Tr} B_{a} P\right) .
$$

To further evaluate this expression, we need a 'good basis' for the generators of $\operatorname{USp}(2 N)$. From

$$
\operatorname{Tr} t_{a} t_{b}=2 \operatorname{Tr} A_{a} A_{b}+2 \Re\left(\operatorname{Tr} B_{a}^{\dagger} B_{b}\right)
$$

we see that $A_{\alpha}=t_{\alpha}, B_{\alpha}=0$ for $\alpha=0, \ldots, N^{2}-1$ and $A_{n+N^{2}}=0, B_{n+N^{2}}=\tilde{t}_{n}$ for $n=0, \ldots, N^{2}+N-1$ is a basis of the kind we want. ${ }^{35}$ Because for this basis $\operatorname{Tr} t_{a} t_{b}$ is again diagonal, i.e. $\operatorname{Tr} t_{a} t_{b}=0$ for $a \neq b$. Since $O$ and $Q$ are hermitian and $P$ is unconstrained, we need for $D_{a}=0$ that

$$
O=Q \quad \text { and } \quad P=-P^{T} .
$$

As argued in section 2.5, these constraints are trivially fulfilled for the constructed orientifold models of the $\mathrm{dP}_{5}$-quiver.

\section{C.2 Larger model I}

Higgsing. After Higgsing, the quiver in figure 6 does not have the required non-chiral matter spectrum. This is a sign that we have to construct new bound states. For this reason, we replace $F_{b}$ by $F_{d}$ defined by [23]

$$
\operatorname{ch}\left(F_{d}\right)=\sum_{i=1}^{8} n_{i}^{(d)} \operatorname{ch}\left(F_{i}\right)=\operatorname{ch}\left(F_{a}\right)+\operatorname{ch}\left(F_{3}\right)+2 \operatorname{ch}\left(F_{b}\right)+\operatorname{ch}\left(F_{8}\right), \quad \operatorname{deg}\left(F_{d}\right)=-6
$$

where

$$
\mathbf{n}^{(d)}=(4,2,4,3,5,4,3,4) .
$$

Looking at the charge vector in terms of the bound states $F_{a}$ and $F_{b}$, the VEVs should be constructible from (2.42) for $2 F_{b}$ and (2.47) for $2 F_{a}$. Indeed, it turns out to be sufficient

\footnotetext{
${ }^{35}$ The $\tilde{t}_{n}$ 's are the $N(N+1)$ generators of the purely real or purely imaginary symmetric matrices, i.e. $\left[\tilde{t}_{n}\right]_{\mu \nu}=\delta_{i_{n} \mu} \delta_{j_{n} \nu}+\delta_{j_{n} \mu} \delta_{i_{n} \nu}$ with $i_{n}, j_{n}$ on of the $N(N+1) / 2$ index tuples $i \leq j \leq N$ and similar for the imaginary ones.
} 
to choose

$$
\begin{array}{rlrl}
\left\langle X_{13}\right\rangle & =\left(\begin{array}{cccc}
a_{1} & 0 & 0 & 0 \\
0 & a_{2} & 0 & 0 \\
0 & 0 & a_{3} & 0 \\
0 & 0 & 0 & a_{4}
\end{array}\right), & \left\langle X_{14}\right\rangle=\left(\begin{array}{ccc}
b_{1} & 0 & 0 \\
0 & b_{2} & 0 \\
0 & 0 & 0 \\
0 & 0 & b_{3}
\end{array}\right), \\
\left\langle X_{23}\right\rangle=\left(\begin{array}{cccc}
0 & 0 & c_{1} & 0 \\
0 & 0 & 0 & 0
\end{array}\right), & \left\langle X_{24}\right\rangle=\left(\begin{array}{ccc}
0 & 0 & 0 \\
0 & 0 & d_{1}
\end{array}\right), \\
\left\langle X_{35}^{T}\right\rangle= & \left(\begin{array}{cccc}
x_{1} & 0 & 0 & 0 \\
0 & x_{2} & 0 & 0 \\
0 & 0 & x_{3} & 0 \\
0 & 0 & 0 & 0 \\
0 & 0 & 0 & 0
\end{array}\right), & \left\langle X_{36}^{T}\right\rangle=\left(\begin{array}{cccc}
y_{1} & 0 & 0 & 0 \\
0 & y_{2} & 0 & 0 \\
0 & 0 & 0 & 0 \\
0 & 0 & 0 & y_{3}
\end{array}\right), \\
\left\langle X_{45}^{T}\right\rangle= & \left(\begin{array}{ccc}
0 & 0 \\
0 & 0 & 0 \\
z_{1} & 0 & z_{4} \\
0 & z_{2} & 0 \\
0 & 0 & z_{3}
\end{array}\right), & \left\langle X_{46}^{T}\right\rangle=\left(\begin{array}{ccc}
0 & 0 & 0 \\
0 & 0 & 0 \\
w_{1} & 0 & 0 \\
0 & w_{2} & 0
\end{array}\right)
\end{array}
$$

where all entries correspond to $2 \times 2$ matrices. We computed the mass matrix for the gauge potentials numerically and confirmed the breaking pattern

$$
\mathrm{U}(8)^{3} \times \mathrm{U}(4)^{2} \times \mathrm{U}(6)^{2} \times \mathrm{U}(10) \rightarrow \mathrm{U}(2) .
$$

We fix the Higgsed quiver to be of the from $\left(2 F_{a}, N F_{3}, M F_{4}, 2 F_{d}, M F_{7}, N F_{8}\right)$. As before, the VEVs to Higgs the full quiver are obtained by embedding the choices (C.11) for $2 F_{d}$ and (2.47) for $2 F_{a}$ into the larger gauge group representations. This leads to

$$
\begin{aligned}
& \left\langle X_{13}\right\rangle=\left(\begin{array}{cccccc}
a_{1} & 0 & 0 & 0 & 0 & \ldots \\
0 & a_{2} & 0 & 0 & 0 & \ldots \\
0 & 0 & a_{3} & 0 & 0 & \ldots \\
0 & 0 & 0 & a_{4} & 0 & \ldots \\
0 & 0 & 0 & 0 & a_{5} & \ldots \\
0 & 0 & 0 & 0 & 0 & \ldots
\end{array}\right), \quad\left\langle X_{14}\right\rangle=\left(\begin{array}{ccccc}
b_{1} & 0 & 0 & 0 & \ldots \\
0 & b_{2} & 0 & 0 & \ldots \\
0 & 0 & 0 & 0 & \ldots \\
0 & 0 & b_{3} & 0 & \ldots \\
0 & 0 & 0 & b_{4} & \ldots \\
0 & 0 & 0 & 0 & \ldots
\end{array}\right), \\
& \left\langle X_{23}\right\rangle=\left(\begin{array}{cccccc}
0 & 0 & c_{1} & 0 & 0 & \ldots \\
0 & 0 & 0 & 0 & 0 & \ldots \\
0 & 0 & 0 & 0 & c_{2} & \ldots \\
0 & 0 & 0 & 0 & c_{3} & \ldots
\end{array}\right), \quad\left\langle X_{24}\right\rangle=\left(\begin{array}{ccccc}
0 & 0 & 0 & 0 & \ldots \\
0 & 0 & d_{1} & 0 & \ldots \\
0 & 0 & 0 & d_{2} & \ldots \\
0 & 0 & 0 & d_{3} & \ldots
\end{array}\right), \\
& \left\langle X_{35}^{T}\right\rangle=\left(\begin{array}{cccccc}
x_{1} & 0 & 0 & 0 & 0 & \ldots \\
0 & x_{2} & 0 & 0 & 0 & \ldots \\
0 & 0 & x_{3} & 0 & 0 & \ldots \\
0 & 0 & 0 & 0 & 0 & \ldots \\
0 & 0 & 0 & 0 & 0 & \ldots \\
0 & 0 & 0 & x_{4} & x_{5} & \ldots
\end{array}\right), \quad\left\langle X_{45}^{T}\right\rangle=\left(\begin{array}{ccccc}
0 & 0 & 0 & 0 & \ldots \\
0 & 0 & 0 & 0 & \ldots \\
z_{1} & 0 & z_{4} & 0 & \ldots \\
0 & z_{2} & 0 & 0 & \ldots \\
0 & 0 & z_{3} & 0 & \ldots \\
0 & 0 & 0 & z_{5} & \ldots
\end{array}\right),
\end{aligned}
$$




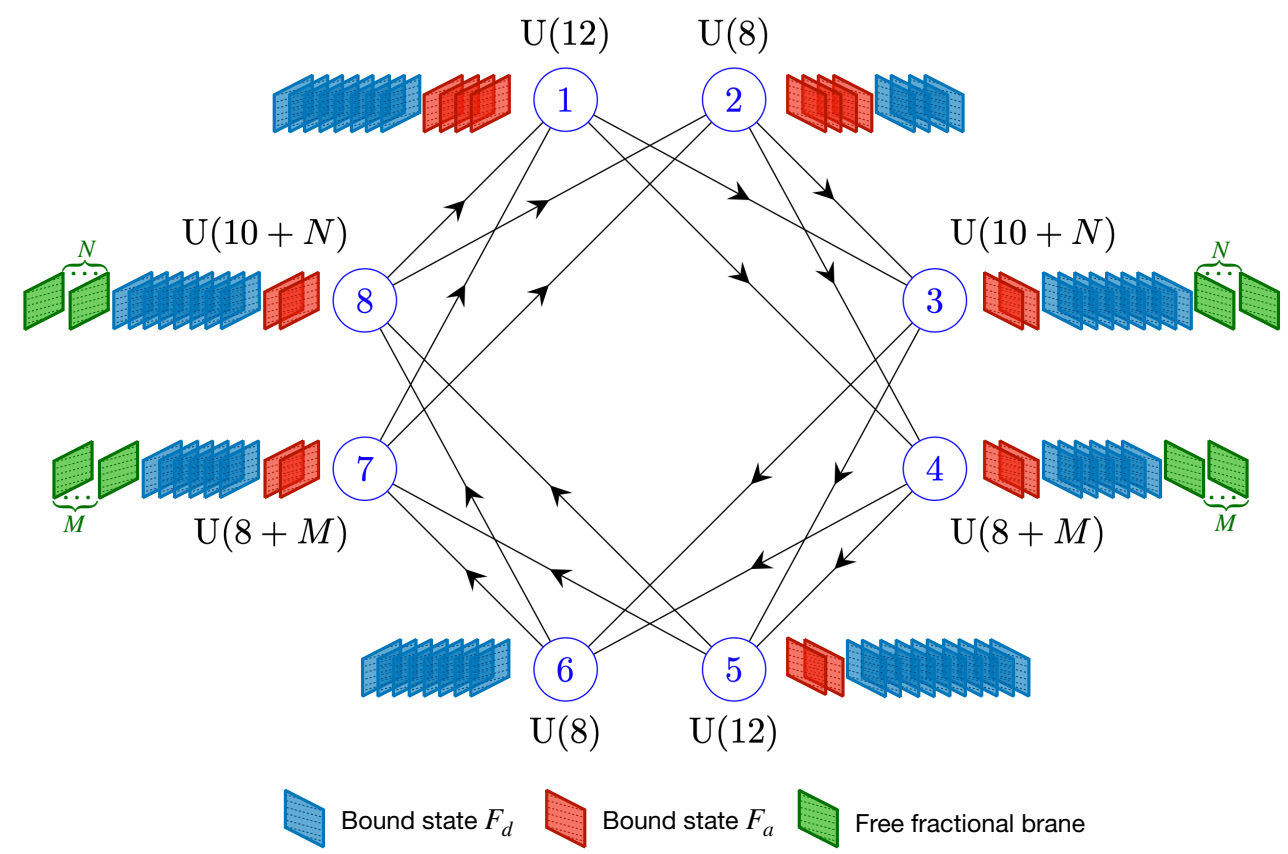

Figure 10. Large model I quiver diagram for a D3-brane at a $\mathrm{dP}_{5}$-singularity with the bound state $2 F_{a}$ indicated in red and $2 F_{d}$ in blue. The green fractional branes do not contributing to any bound state.

$$
\left\langle X_{36}^{T}\right\rangle=\left(\begin{array}{cccccc}
y_{1} & 0 & 0 & 0 & 0 & \ldots \\
0 & y_{2} & 0 & 0 & 0 & \ldots \\
0 & 0 & 0 & 0 & 0 & \ldots \\
0 & 0 & y_{3} & y_{4} & 0 & \ldots
\end{array}\right), \quad\left\langle X_{46}^{T}\right\rangle=\left(\begin{array}{ccccc}
0 & 0 & 0 & 0 & \ldots \\
0 & 0 & 0 & 0 & \ldots \\
w_{1} & 0 & 0 & 0 & \ldots \\
0 & w_{2} & w_{3} & 0 & \ldots
\end{array}\right)
$$

where as usual all entries are $2 \times 2$ matrices and the ... on the left (right) correspond to $N(M)$ zeros. As shown in section 2.5, this leads to the correct spectrum with a quiver generated by multiplicities $(12,8,(10+N),(6+M), 12,8,(6+M),(10+N))$ of fractional branes $\left\{F_{1}, \ldots, F_{8}\right\}$ which is shown in figure 10 . The corresponding bound states $F_{a}$ and $F_{d}$ are depicted in red and blue respectively. The quiver after Higgsing together with the field content is shown in figure 8 .

$D$-flatness conditions. Let us check the non-abelian $D$-term conditions. Plugging the VEVs (C.13) into the expression for the non-abelian D-terms (2.62) gives rise to two nontrivial conditions. The first is given by

$$
\sum_{i=1}^{5}\left(\left|a_{i}\right|^{2}-\left|x_{i}\right|^{2}\right)+\left|c_{1}\right|^{2}+\left|c_{2}\right|^{2}+\left|c_{3}\right|^{2}-\left|y_{1}\right|^{2}-\left|y_{2}\right|^{2}-\left|y_{3}\right|^{2}-\left|y_{4}\right|^{2}=0
$$

which can be solved by choosing

$$
\begin{aligned}
& \left|a_{1}\right|^{2}=\sum_{i=1}^{4}\left|x_{i}\right|^{2}+\left|y_{1}\right|^{2}+\left|y_{2}\right|^{2}+\left|y_{3}\right|^{2}+\left|y_{4}\right|^{2} \\
& \left|x_{5}\right|^{2}=\sum_{i=2}^{5}\left|a_{i}\right|^{2}+\left|c_{1}\right|^{2}+\left|c_{2}\right|^{2}+\left|c_{3}\right|^{2} .
\end{aligned}
$$


Furthermore,we find the constraint

$$
\sum_{i=1}^{4}\left(\left|b_{i}\right|^{2}-\left|z_{i}\right|^{2}\right)+\left|d_{1}\right|^{2}+\left|d_{2}\right|^{2}+\left|d_{3}\right|^{2}-\left|z_{5}\right|^{2}-\left|w_{1}\right|^{2}-\left|w_{2}\right|^{2}-\left|w_{3}\right|^{2}=0
$$

which can be solved by choosing

$$
\begin{aligned}
& \left|b_{1}\right|^{2}=\sum_{i=1}^{4}\left|z_{i}\right|^{2}+\left|w_{1}\right|^{2}+\left|w_{2}\right|^{2}+\left|w_{3}\right|^{2} \\
& \left|z_{5}\right|^{2}=\sum_{i=2}^{4}\left|b_{i}\right|^{2}+\left|d_{1}\right|^{2}+\left|d_{2}\right|^{2}+\left|d_{3}\right|^{2}
\end{aligned}
$$

We have 4 real conditions on a total of 32 complex parameters leaving us with 30 complex parameters.

For the abelian $D$-terms, we obtain from (2.67)

$$
\begin{aligned}
& D_{1}=D_{2}=D_{5}=D_{6}=0 \\
& D_{8}=-D_{3}, \quad D_{7}=-D_{8}, \\
& D_{3}=-2\left(\sum_{i=1}^{5}\left(\left|a_{i}\right|^{2}-\left|x_{i}\right|^{2}\right)+\left|c_{1}\right|^{2}+\left|c_{2}\right|^{2}+\left|c_{3}\right|^{2}-\left|y_{1}\right|^{2}-\left|y_{2}\right|^{2}-\left|y_{3}\right|^{2}-\left|y_{4}\right|^{2}\right) \\
& D_{4}=-2\left(\sum_{i=1}^{4}\left(\left|b_{i}\right|^{2}-\left|z_{i}\right|^{2}\right)+\left|d_{1}\right|^{2}+\left|d_{2}\right|^{2}+\left|d_{3}\right|^{2}-\left|z_{5}\right|^{2}-\left|w_{1}\right|^{2}-\left|w_{2}\right|^{2}-\left|w_{3}\right|^{2}\right) .
\end{aligned}
$$

Again, all abelian D-terms vanish upon utilising (C.15) and (C.17). The conclusion are therefore the same as for the small version of model I discussed in section 2.7.

\section{C.3 Higgsing model II}

Let us briefly discuss the Higgsing procedure for Model II defined by the orientifold involution (2.30). We consider the following bound states [23]

$$
\begin{aligned}
\operatorname{ch}\left(F_{a}\right) & =\sum_{i=1}^{8} N_{i}^{(a)} \operatorname{ch}\left(F_{i}\right)=\left(3,2 H-2 E_{1}+E_{2}-E_{3}+E_{4}+E_{5}, 1\right) \\
\mathbf{n}^{(a)} & =(4,1,2,2,1,1,2,2), \\
\operatorname{ch}\left(F_{b}\right) & =\sum_{i=1}^{8} N_{i}^{(b)} \operatorname{ch}\left(F_{i}\right)=-\left(3,-E_{1}-2 E_{2}+3 E_{3}+3 E_{4}+3 E_{5}, 6\right) \\
\mathbf{n}^{(b)} & =(2,1,3,3,0,6,3,3), \\
\operatorname{ch}\left(F_{b^{\prime}}\right) & =\sum_{i=1}^{8} N_{i}^{\left(b^{\prime}\right)} \operatorname{ch}\left(F_{i}\right)=\left(3,2 H-2 E_{1}+E_{2}-E_{3}+E_{4}+E_{5}, 1\right) \\
\mathbf{n}^{\left(b^{\prime}\right)} & =(2,1,3,3,6,0,3,3) .
\end{aligned}
$$


For the bound state $2 F_{a}$, we choose

$$
\begin{aligned}
& \left\langle X_{13}\right\rangle=\left(\begin{array}{cccc}
a_{1} & 0 & 0 & 0 \\
0 & a_{2} & 0 & 0 \\
0 & 0 & 0 & 0 \\
0 & 0 & 0 & 0 \\
0 & 0 & a_{1} & 0 \\
0 & 0 & 0 & a_{2} \\
0 & 0 & 0 & 0 \\
0 & 0 & 0 & 0
\end{array}\right),\left\langle X_{14}\right\rangle=\left(\begin{array}{cccc}
0 & 0 & 0 & 0 \\
0 & 0 & 0 & 0 \\
a_{2} & 0 & 0 & 0 \\
0 & a_{1} & 0 & 0 \\
0 & 0 & 0 & 0 \\
0 & 0 & 0 & 0 \\
0 & 0 & a_{2} & 0 \\
0 & 0 & 0 & a_{1}
\end{array}\right) \\
& \left\langle X_{23}\right\rangle=\left(\begin{array}{cccc}
a_{3} & 0 & 0 & 0 \\
0 & 0 & a_{3} & 0
\end{array}\right), \quad\left\langle X_{24}\right\rangle=\left(\begin{array}{cccc}
0 & a_{3} & 0 & 0 \\
0 & 0 & 0 & a_{3}
\end{array}\right) \\
& \left\langle X_{36}\right\rangle=\left(\begin{array}{cc}
0 & 0 \\
0 & a_{5} \\
0 & 0 \\
a_{5} & 0
\end{array}\right), \quad\left\langle X_{46}\right\rangle=\left(\begin{array}{cc}
a_{4} & 0 \\
0 & 0 \\
0 & a_{4} \\
0 & 0
\end{array}\right) \\
& \left\langle X_{35}\right\rangle=\left(\begin{array}{cc}
a_{4} & 0 \\
0 & 0 \\
0 & a_{4} \\
0 & 0
\end{array}\right), \quad\left\langle X_{45}\right\rangle=\left(\begin{array}{cc}
0 & 0 \\
a_{5} & 0 \\
0 & 0 \\
0 & a_{5}
\end{array}\right)
\end{aligned}
$$

For $F_{b}+F_{b^{\prime}}$ we use

$$
\begin{aligned}
& \left\langle X_{13}\right\rangle=\left\langle X_{14}\right\rangle=\left(\begin{array}{cccccc}
e_{1} & 0 & 0 & 0 & 0 & 0 \\
0 & e_{1} & 0 & 0 & 0 & 0 \\
0 & 0 & 0 & e_{1} & 0 & 0 \\
0 & 0 & 0 & 0 & e_{1} & 0
\end{array}\right) \\
& \left\langle X_{23}\right\rangle=\left\langle X_{24}\right\rangle=\left(\begin{array}{cccccc}
0 & 0 & e_{1} & 0 & 0 & 0 \\
0 & 0 & 0 & 0 & 0 & e_{1}
\end{array}\right) \\
& \left\langle X_{35}\right\rangle=\left(\begin{array}{cccccc}
e_{1} & 0 & 0 & 0 & 0 & 0 \\
0 & e_{1} & 0 & 0 & 0 & 0 \\
0 & 0 & e_{1} & 0 & 0 & 0 \\
0 & 0 & 0 & 0 & 0 & 0 \\
0 & 0 & 0 & 0 & 0 & 0 \\
0 & 0 & 0 & 0 & 0 & 0
\end{array}\right), \\
& \left\langle X_{45}\right\rangle=\left(\begin{array}{cccccc}
0 & 0 & 0 & e_{1} & 0 & 0 \\
0 & 0 & 0 & 0 & e_{1} & 0 \\
0 & 0 & 0 & 0 & 0 & e_{1} \\
0 & 0 & 0 & 0 & 0 & 0 \\
0 & 0 & 0 & 0 & 0 & 0 \\
0 & 0 & 0 & 0 & 0 & 0
\end{array}\right) \\
& \left\langle X_{35}\right\rangle=\left(\begin{array}{cccccc}
0 & 0 & 0 & 0 & 0 & 0 \\
0 & 0 & 0 & 0 & 0 & 0 \\
0 & 0 & 0 & 0 & 0 & 0 \\
0 & 0 & 0 & 0 & 0 & e_{1} \\
0 & 0 & 0 & e_{1} & 0 & 0 \\
0 & 0 & 0 & 0 & e_{1} & 0
\end{array}\right), \\
& \left\langle X_{45}\right\rangle=\left(\begin{array}{cccccc}
0 & 0 & 0 & 0 & 0 & 0 \\
0 & 0 & 0 & 0 & 0 & 0 \\
0 & 0 & 0 & 0 & 0 & 0 \\
0 & 0 & e_{1} & 0 & 0 & 0 \\
e_{1} & 0 & 0 & 0 & 0 & 0 \\
0 & e_{1} & 0 & 0 & 0 & 0
\end{array}\right)
\end{aligned}
$$




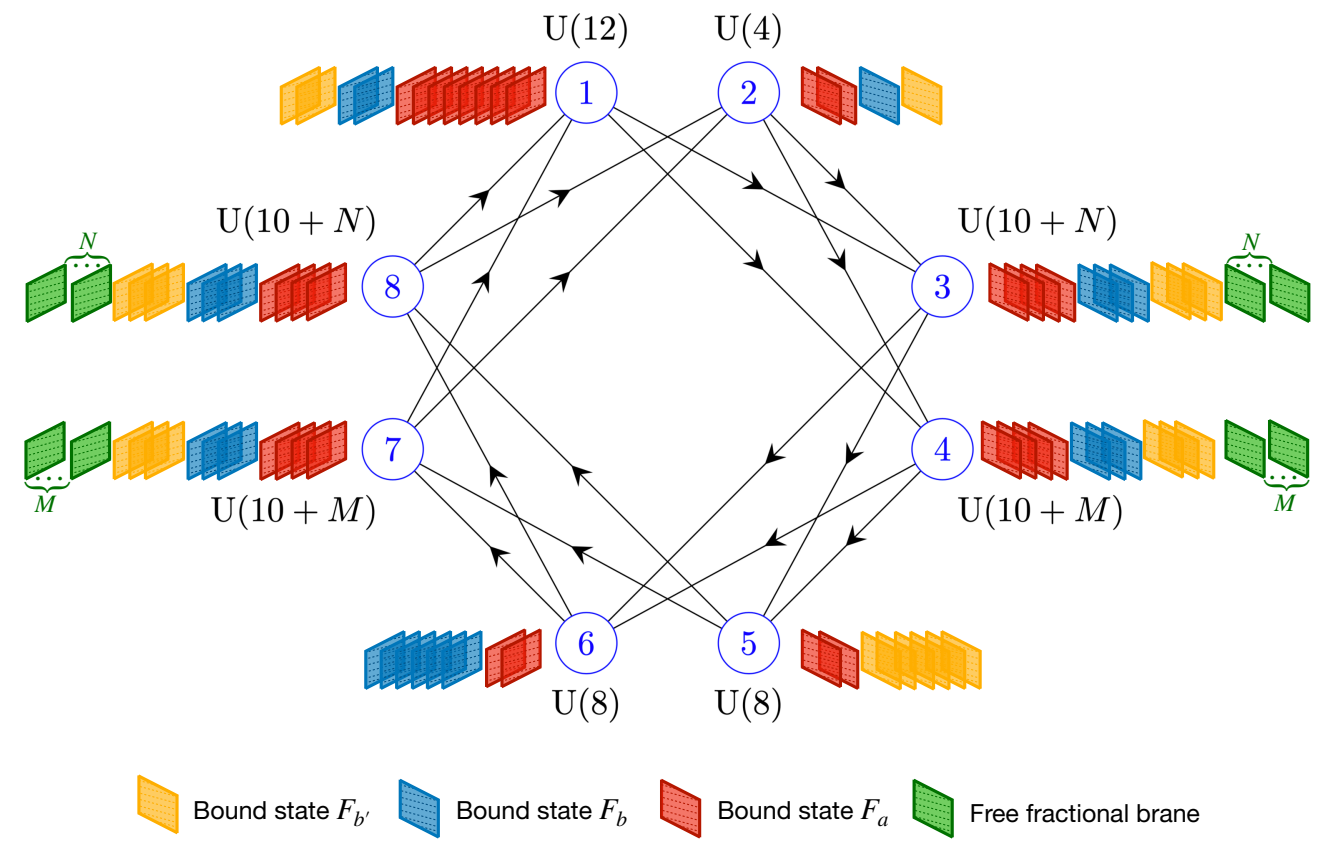

Figure 11. Model II quiver diagram $\mathrm{dP}_{5}$ with the bound states $2 F_{a}$ (red), $F_{b}$ (blue) and $F_{b^{\prime}}$ (orange). Green fractional branes do not participate in any bound state.

We showed that both lead to the expected breaking patterns:

$$
\begin{aligned}
2 F_{a}: & \mathrm{U}(8) \times \mathrm{U}(2)^{3} \times \mathrm{U}(4)^{4} \rightarrow \mathrm{U}(2), \\
F_{b}+F_{b^{\prime}}: & \mathrm{U}(4) \times \mathrm{U}(2) \times \mathrm{U}(6)^{6} \rightarrow \mathrm{U}(1) \times \mathrm{U}(1) .
\end{aligned}
$$

Altogether, we construct the Higgsed quiver $\left(2 F_{a}, N F_{3}, M F_{4}, F_{b}+F_{b^{\prime}}, M F_{7}, N F_{8}\right)$ which is obtained from $\left(12 F_{1}, 4 F_{2}, 13 F_{3}, 11 F_{4}, 8 F_{5}, 8 F_{6}, 11 F_{7}, 13 F_{8}\right)$, see figure 11 . Here, the bound state $2 F_{a}$ is depicted in red, $F_{b}$ in blue and $F_{b^{\prime}}$ in green, while black dots represent fractional branes not participating in any bound state. The Higgsed quiver is shown in figure 12. After performing the orientifolding, one ends up with the quiver on the right of figure 12 for $N=3$ and $M=1$. The $\mathrm{U}(1)$ at the bottom node comes from identifying two different nodes in the covering quiver. The field content is computed from

$$
\begin{aligned}
G & =\mathrm{U}(12) \times \mathrm{U}(4) \times(\mathrm{U}(10+N) \times \mathrm{U}(10+M) \times \mathrm{U}(8))^{2}, \\
\operatorname{dim}(G) & =688+40(N+M)+2\left(N^{2}+M^{2}\right), \\
H & =\mathrm{U}(2) \times(\mathrm{U}(1) \times \mathrm{U}(N) \times \mathrm{U}(M))^{2}, \\
\operatorname{dim}(H) & =6+2\left(N^{2}+M^{2}\right), \\
\operatorname{dim}(G / H) & =682+40(N+M), \\
N_{\text {chiral }} & =1280+64(N+M), \\
N_{\text {chiral }}^{\text {Higged }} & =24(N+M), \\
N_{\text {chiral }}-\operatorname{dim}(G / H) & =598+N_{\text {chiral }}^{\text {Higgsed }} .
\end{aligned}
$$


Higgsed quiver

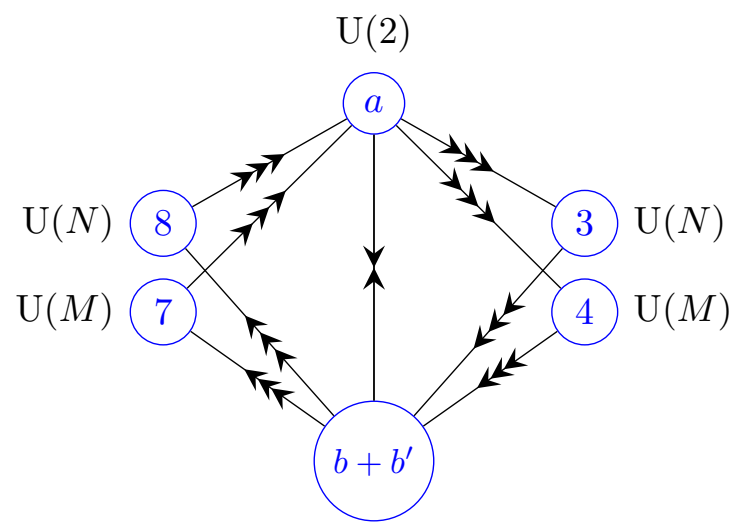

$\mathrm{U}(1) \times \mathrm{U}(1)$
Higgsed and orientifolded quiver

$\mathrm{SU}(2)$

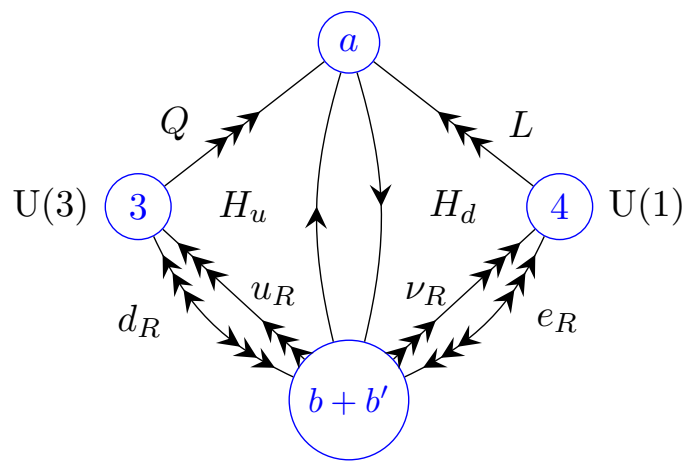

$\mathrm{U}(1)$

Figure 12. Left: Higgsed $\mathrm{dP}_{5}$ quiver diagram for Model II obtained from the quiver in figure 11. Right: orientifolded quiver diagram for $N=3$ and $M=1$ via the involution (2.30).

We find 598 complex scalars in bi-fundamentals between $\mathrm{U}(2)$ and one of the U(1)'s or between the two $\mathrm{U}(1)$ 's. There is no non-chiral matter between either $\mathrm{U}(N)$ or $\mathrm{U}(M)$ and $\mathrm{U}(2)(\mathrm{U}(1))$.

\section{Line orientifold of the complex cone over $\mathrm{dP}_{5}$}

Geometry. This is a quick review of some of the properties of the complex cone over $\mathrm{dP}_{5}$. At some specific loci in moduli space it can be described as a $\mathbb{Z}_{2} \times \mathbb{Z}_{2}$ orbifold of the conifold, which can be described as a particular phase of the following GLSM

$$
\begin{array}{c|cccccc} 
& z_{1} & z_{2} & z_{3} & z_{4} & u & t \\
\hline \mathbb{C}_{1}^{*} & 1 & 1 & 0 & 0 & 0 & -2 \\
\mathbb{C}_{2}^{*} & 0 & 0 & 1 & 1 & 0 & -2 \\
\mathbb{C}_{3}^{*} & 1 & 0 & 1 & 0 & -2 & 0
\end{array}
$$

The $\mathbb{Z}_{2} \times \mathbb{Z}_{2}$ action can be seen easily by going to a phase with $\xi_{1}=\xi_{2}<0$ and $\xi_{3}<0$, and gauge fixing $t=u=1$. This leaves a $\mathbb{Z}_{2} \times \mathbb{Z}_{2}$ acting on the conifold coordinates $z_{i}$ as

$$
\begin{aligned}
& \sigma_{1}:\left(z_{1}, z_{2}, z_{3}, z_{4}\right) \rightarrow\left(z_{1}, z_{2},-z_{3},-z_{4}\right) \\
& \sigma_{2}:\left(z_{1}, z_{2}, z_{3}, z_{4}\right) \rightarrow\left(-z_{1}, z_{2},-z_{3}, z_{4}\right) .
\end{aligned}
$$

The toric diagram is shown in figure 13 .

The monomial ring is generated by the gauge invariants

$$
X=\left(z_{1} z_{3}\right)^{2} t u^{2} ; \quad Y=\left(z_{2} z_{4}\right)^{2} t ; \quad C=z_{1} z_{2} z_{3} z_{4} t u ; \quad Z=\left(z_{1} z_{4}\right)^{2} t u ; \quad W=\left(z_{2} z_{3}\right)^{2} t u
$$

which satisfy

$$
X Y=Z W=C^{2}
$$

which is manifestly a double cover of the conifold branched over the Cartier divisors $X=0$, $Y=0, Z=0$ and $W=0$. 


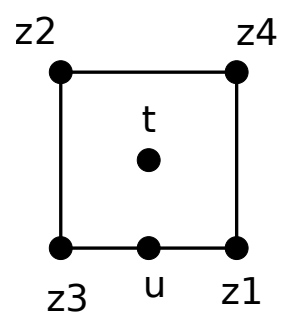

Figure 13. Toric data for the toric $\mathrm{dP}_{5}$.

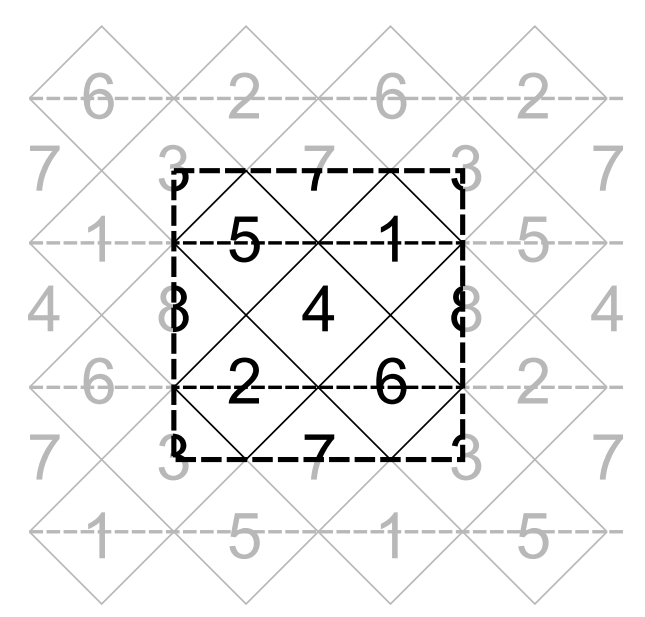

Figure 14. Line orientifold of the dimer model for the $\mathbb{Z}_{2} \times \mathbb{Z}_{2}$ orbifold of the conifold described in text. We have highlighted a fundamental cell, and denoted dashed line the involution in which we are interested.

Orientifold. We are interested in the line orientifold shown in figure 14. This is in fact closely related to the quotient studied in detail in $[83,128,129]$, so we can easily read the results from there. Using the techniques in [128] we find that the orientifold action on the fundamental mesons, identified with the geometric fields introduced above, is given by

$$
(X, Y, Z, W, C) \rightarrow(\epsilon X, \epsilon Y, W, Z, C)
$$

with $\epsilon$ the product of the signs of the fixed lines. Namely, if the two fixed lines have the same sign we have that $\epsilon=+1$, and the fixed locus is at $Z=W$, giving rise to a noncompact O7 plane. (As described in [129] the sign of the non-compact plane is determined by the projection, with USp projection corresponding to the $\mathrm{O}^{-}$case.) If the two fixed lines have opposite sign, $\epsilon=-1$, corresponding to opposite choices of Chan-Paton factor on the two fixed lines, the fixed locus is at $X=Y=Z=W=C=0$.

The two projections of interest to us can be described in terms of the toric coordinates by

$$
\left(z_{1}, z_{2}, z_{3}, z_{4}, u, t\right) \rightarrow\left(-z_{3}, z_{4}, z_{1},-z_{2}, \epsilon u, \epsilon t\right) .
$$

The action on the toric diagram is shown on the left of figure 15 .

We see that there are some resolutions of the $\mathrm{dP}_{5}$ singularity that are compatible with the involution, an example is on the right of figure 15 . 

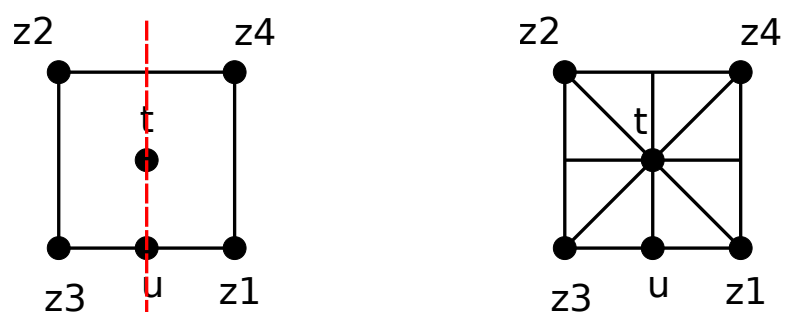

Figure 15. Left: the involution acts on the toric diagram by reflecting along the dashed red line. Right: a resolution of the toric singularity compatible with the involution, at least at the level of the toric diagram.

Open Access. This article is distributed under the terms of the Creative Commons Attribution License (CC-BY 4.0), which permits any use, distribution and reproduction in any medium, provided the original author(s) and source are credited.

\section{References}

[1] G. Aldazabal, L.E. Ibáñez, F. Quevedo and A.M. Uranga, D-branes at singularities: A Bottom up approach to the string embedding of the standard model, JHEP 08 (2000) 002 [hep-th/0005067] [INSPIRE].

[2] R. Blumenhagen, L. Görlich, B. Körs and D. Lüst, Noncommutative compactifications of type-I strings on tori with magnetic background flux, JHEP 10 (2000) 006 [hep-th/0007024] [INSPIRE].

[3] G. Aldazabal, S. Franco, L.E. Ibáñez, R. Rabadán and A.M. Uranga, D $=4$ chiral string compactifications from intersecting branes, J. Math. Phys. 42 (2001) 3103 [hep-th/0011073] [INSPIRE].

[4] R. Blumenhagen, B. Körs, D. Lüst and T. Ott, The standard model from stable intersecting brane world orbifolds, Nucl. Phys. B 616 (2001) 3 [hep-th/0107138] [INSPIRE].

[5] M. Cvetič, G. Shiu and A.M. Uranga, Three family supersymmetric standard-like models from intersecting brane worlds, Phys. Rev. Lett. 87 (2001) 201801 [hep-th/0107143] [INSPIRE].

[6] J.P. Conlon, A. Maharana and F. Quevedo, Towards Realistic String Vacua, JHEP 05 (2009) 109 [arXiv: 0810.5660] [INSPIRE].

[7] R. Blumenhagen, V. Braun, T.W. Grimm and T. Weigand, GUTs in Type IIB Orientifold Compactifications, Nucl. Phys. B $\mathbf{8 1 5}$ (2009) 1 [arXiv:0811.2936] [INSPIRE].

[8] R. Donagi and M. Wijnholt, Model Building with F-theory, Adv. Theor. Math. Phys. 15 (2011) 1237 [arXiv: 0802.2969] [INSPIRE].

[9] C. Beasley, J.J. Heckman and C. Vafa, GUTs and Exceptional Branes in F-theory - I, JHEP 01 (2009) 058 [arXiv:0802.3391] [INSPIRE].

[10] C. Beasley, J.J. Heckman and C. Vafa, GUTs and Exceptional Branes in F-theory - II: Experimental Predictions, JHEP 01 (2009) 059 [arXiv:0806.0102] [INSPIRE].

[11] R. Donagi and M. Wijnholt, Breaking GUT Groups in F-theory, Adv. Theor. Math. Phys. 15 (2011) 1523 [arXiv:0808.2223] [InSPIRE]. 
[12] M. Cvetič, J. Halverson, L. Lin, M. Liu and J. Tian, Quadrillion F-Theory Compactifications with the Exact Chiral Spectrum of the Standard Model, Phys. Rev. Lett. 123 (2019) 101601 [arXiv: 1903.00009] [INSPIRE].

[13] S. Franco and A.M. Uranga, Dynamical SUSY breaking at meta-stable minima from D-branes at obstructed geometries, JHEP 06 (2006) 031 [hep-th/0604136] [INSPIRE].

[14] S. Franco, Bipartite Field Theories: from D-brane Probes to Scattering Amplitudes, JHEP 11 (2012) 141 [arXiv:1207.0807] [INSPIRE].

[15] S. Franco and A. Uranga, Bipartite Field Theories from D-branes, JHEP 04 (2014) 161 [arXiv:1306.6331] [INSPIRE].

[16] M. Bianchi, G. Inverso, J.F. Morales and D. Ricci Pacifici, Unoriented Quivers with Flavour, JHEP 01 (2014) 128 [arXiv:1307.0466] [INSPIRE].

[17] M. Bianchi, D. Bufalini, S. Mancani and F. Riccioni, Mass deformations of unoriented quiver theories, JHEP 07 (2020) 015 [arXiv: 2003.09620] [inSPIRE].

[18] M. Cicoli, S. Krippendorf, C. Mayrhofer, F. Quevedo and R. Valandro, D-Branes at del Pezzo Singularities: Global Embedding and Moduli Stabilisation, JHEP 09 (2012) 019 [arXiv:1206.5237] [INSPIRE].

[19] M. Cicoli, S. Krippendorf, C. Mayrhofer, F. Quevedo and R. Valandro, D3/D7 Branes at Singularities: Constraints from Global Embedding and Moduli Stabilisation, JHEP 07 (2013) 150 [arXiv: 1304.0022] [INSPIRE].

[20] M. Cicoli, S. Krippendorf, C. Mayrhofer, F. Quevedo and R. Valandro, The Web of D-branes at Singularities in Compact Calabi-Yau Manifolds, JHEP 05 (2013) 114 [arXiv: 1304.2771] [INSPIRE].

[21] M. Cicoli, D. Klevers, S. Krippendorf, C. Mayrhofer, F. Quevedo and R. Valandro, Explicit de Sitter Flux Vacua for Global String Models with Chiral Matter, JHEP 05 (2014) 001 [arXiv: 1312.0014] [INSPIRE].

[22] M. Cicoli, I. Garcìa-Etxebarria, C. Mayrhofer, F. Quevedo, P. Shukla and R. Valandro, Global Orientifolded Quivers with Inflation, JHEP 11 (2017) 134 [arXiv:1706.06128] [INSPIRE].

[23] M. Wijnholt, Geometry of Particle Physics, Adv. Theor. Math. Phys. 13 (2009) 947 [hep-th/0703047] [INSPIRE].

[24] L.E. Ibáñez, F. Marchesano and R. Rabadán, Getting just the standard model at intersecting branes, JHEP 11 (2001) 002 [hep-th/0105155] [INSPIRE].

[25] P. Anastasopoulos, T.P.T. Dijkstra, E. Kiritsis and A.N. Schellekens, Orientifolds, hypercharge embeddings and the Standard Model, Nucl. Phys. B 759 (2006) 83 [hep-th/0605226] [INSPIRE].

[26] D. Berenstein and S. Pinansky, The Minimal Quiver Standard Model, Phys. Rev. D 75 (2007) 095009 [hep-th/0610104] [INSPIRE].

[27] J.C. Pati and A. Salam, Lepton Number as the Fourth Color, Phys. Rev. D 10 (1974) 275 [Erratum ibid. 11 (1975) 703] [INSPIRE].

[28] R.N. Mohapatra and J.C. Pati, Left-Right Gauge Symmetry and an Isoconjugate Model of CP-violation, Phys. Rev. D 11 (1975) 566 [inSPIRE]. 
[29] G. Senjanović and R.N. Mohapatra, Exact Left-Right Symmetry and Spontaneous Violation of Parity, Phys. Rev. D 12 (1975) 1502 [INSPIRE].

[30] M. Cicoli, M. Kreuzer and C. Mayrhofer, Toric K3-Fibred Calabi-Yau Manifolds with del Pezzo Divisors for String Compactifications, JHEP 02 (2012) 002 [arXiv:1107.0383] [INSPIRE].

[31] M. Kreuzer and H. Skarke, Complete classification of reflexive polyhedra in four-dimensions, Adv. Theor. Math. Phys. 4 (2002) 1209 [hep-th/0002240] [InSPIRE].

[32] D.S. Freed and E. Witten, Anomalies in string theory with D-branes, Asian J. Math. 3 (1999) 819 [hep-th/9907189] [INSPIRE].

[33] R. Donagi, S. Katz and E. Sharpe, Spectra of D-branes with Higgs vevs, Adv. Theor. Math. Phys. 8 (2004) 813 [hep-th/0309270] [INSPIRE].

[34] S. Cecotti, C. Cordova, J.J. Heckman and C. Vafa, T-Branes and Monodromy, JHEP 07 (2011) 030 [arXiv: 1010.5780] [INSPIRE].

[35] M. Cicoli, F. Quevedo and R. Valandro, de Sitter from T-branes, JHEP 03 (2016) 141 [arXiv: 1512.04558] [INSPIRE].

[36] E. Witten, Nonperturbative superpotentials in string theory, Nucl. Phys. B 474 (1996) 343 [hep-th/9604030] [INSPIRE].

[37] V. Balasubramanian, P. Berglund, J.P. Conlon and F. Quevedo, Systematics of moduli stabilisation in Calabi-Yau flux compactifications, JHEP 03 (2005) 007 [hep-th/0502058] [INSPIRE].

[38] J.P. Conlon and F.G. Pedro, Moduli Redefinitions and Moduli Stabilisation, JHEP 06 (2010) 082 [arXiv: 1003.0388] [INSPIRE].

[39] J.P. Conlon and F. Quevedo, Kähler moduli inflation, JHEP 01 (2006) 146 [hep-th/0509012] [INSPIRE].

[40] M. Cicoli and A. Mazumdar, Reheating for Closed String Inflation, JCAP 09 (2010) 025 [arXiv: 1005.5076] [INSPIRE].

[41] M. Cicoli and A. Mazumdar, Inflation in string theory: A Graceful exit to the real world, Phys. Rev. D 83 (2011) 063527 [arXiv: 1010.0941] [InSPIRE].

[42] R. Allahverdi, I. Broeckel, M. Cicoli and J.K. Osiński, Superheavy dark matter from string theory, JHEP 02 (2021) 026 [arXiv: 2010.03573] [INSPIRE].

[43] R. Allahverdi, M. Cicoli and F. Muia, Affleck-Dine Baryogenesis in Type IIB String Models, JHEP 06 (2016) 153 [arXiv:1604.03120] [INSPIRE].

[44] M. Cicoli, J.P. Conlon and F. Quevedo, Dark radiation in LARGE volume models, Phys. Rev. D 87 (2013) 043520 [arXiv: 1208.3562] [INSPIRE].

[45] T. Higaki and F. Takahashi, Dark Radiation and Dark Matter in Large Volume Compactifications, JHEP 11 (2012) 125 [arXiv:1208.3563] [INSPIRE].

[46] M. Cicoli and F. Muia, General Analysis of Dark Radiation in Sequestered String Models, JHEP 12 (2015) 152 [arXiv: 1511.05447] [INSPIRE].

[47] P. Wilson, The kähler cone on calabi-yau threefolds, Invent. Math. 107 (1992) 561.

[48] Y. Hayakawa, Degenaration of Calabi-Yau Manifold with W-P Metric, alg-geom/9507016. 
[49] P.S. Aspinwall, An $N=2$ dual pair and a phase transition, Nucl. Phys. B 460 (1996) 57 [hep-th/9510142] [INSPIRE].

[50] M. Bershadsky, C. Vafa and V. Sadov, D strings on D manifolds, Nucl. Phys. B 463 (1996) 398 [hep-th/9510225] [INSPIRE].

[51] S.H. Katz, D.R. Morrison and M.R. Plesser, Enhanced gauge symmetry in type-II string theory, Nucl. Phys. B 477 (1996) 105 [hep-th/9601108] [InSPIRE].

[52] T.-m. Chiang, B.R. Greene, M. Gross and Y. Kanter, Black hole condensation and the web of Calabi-Yau manifolds, Nucl. Phys. B Proc. Suppl. 46 (1996) 82 [hep-th/9511204] [INSPIRE].

[53] D.R. Morrison and C. Vafa, Compactifications of F-theory on Calabi-Yau threefolds. 2, Nucl. Phys. B 476 (1996) 437 [hep-th/9603161] [inSPIRE].

[54] M.R. Douglas, S.H. Katz and C. Vafa, Small instantons, Del Pezzo surfaces and type-I-prime theory, Nucl. Phys. B 497 (1997) 155 [hep-th/9609071] [InSPIRE].

[55] H. Grauert, Über Modifikationen und exzeptionelle analytische Mengen, Math. Ann. 146 (1962) 331.

[56] C. Cordova, Decoupling Gravity in F-theory, Adv. Theor. Math. Phys. 15 (2011) 689 [arXiv:0910.2955] [INSPIRE].

[57] E.R. Sharpe, D-branes, derived categories, and Grothendieck groups, Nucl. Phys. B 561 (1999) 433 [hep-th/9902116] [INSPIRE].

[58] M.R. Douglas, D-branes, categories and N=1 supersymmetry, J. Math. Phys. 42 (2001) 2818 [hep-th/0011017] [INSPIRE].

[59] P.S. Aspinwall, D-branes on Calabi-Yau manifolds, in Theoretical Advanced Study Institute in Elementary Particle Physics (TASI 2003): Recent Trends in String Theory, (2004) [DOI] [hep-th/0403166] [INSPIRE].

[60] M.R. Douglas, B. Fiol and C. Romelsberger, Stability and BPS branes, JHEP 09 (2005) 006 [hep-th/0002037] [INSPIRE].

[61] M.R. Douglas, B. Fiol and C. Romelsberger, The Spectrum of BPS branes on a noncompact Calabi-Yau, JHEP 09 (2005) 057 [hep-th/0003263] [INSPIRE].

[62] F. Cachazo, B. Fiol, K.A. Intriligator, S. Katz and C. Vafa, A Geometric unification of dualities, Nucl. Phys. B 628 (2002) 3 [hep-th/0110028] [INSPIRE].

[63] M. Wijnholt, Large volume perspective on branes at singularities, Adv. Theor. Math. Phys. 7 (2003) 1117 [hep-th/0212021] [INSPIRE].

[64] C.P. Herzog, Seiberg duality is an exceptional mutation, JHEP 08 (2004) 064 [hep-th/0405118] [INSPIRE].

[65] P.S. Aspinwall and I.V. Melnikov, D-branes on vanishing del Pezzo surfaces, JHEP 12 (2004) 042 [hep-th/0405134] [INSPIRE].

[66] C.P. Herzog and R.L. Karp, Exceptional collections and D-branes probing toric singularities, JHEP 02 (2006) 061 [hep-th/0507175] [INSPIRE].

[67] A. Hanany, C.P. Herzog and D. Vegh, Brane tilings and exceptional collections, JHEP 07 (2006) 001 [hep-th/0602041] [INSPIRE]. 
[68] B.V. Karpov and D.Y. Nogin, Three-block exceptional collections over Del Pezzo surfaces, Izv. Math. 62 (1998) 429 [alg-geom/9703027].

[69] C.P. Herzog, Exceptional collections and del Pezzo gauge theories, JHEP 04 (2004) 069 [hep-th/0310262] [INSPIRE].

[70] C.P. Herzog and J. Walcher, Dibaryons from exceptional collections, JHEP 09 (2003) 060 [hep-th/0306298] [INSPIRE].

[71] H. Verlinde and M. Wijnholt, Building the standard model on a D3-brane, JHEP 01 (2007) 106 [hep-th/0508089] [INSPIRE].

[72] M. Buican, D. Malyshev, D.R. Morrison, H. Verlinde and M. Wijnholt, D-branes at Singularities, Compactification, and Hypercharge, JHEP 01 (2007) 107 [hep-th/0610007] [INSPIRE].

[73] L.E. Ibáñez, R. Rabadán and A.M. Uranga, Anomalous U(1)'s in type-I and type IIB $D=4, N=1$ string vacua, Nucl. Phys. B 542 (1999) 112 [hep-th/9808139] [INSPIRE].

[74] K.D. Kennaway, Brane Tilings, Int. J. Mod. Phys. A 22 (2007) 2977 [arXiv:0706.1660] [INSPIRE].

[75] D. Malyshev and H. Verlinde, D-branes at singularities and string phenomenology, Nucl. Phys. B Proc. Suppl. 171 (2007) 139 [arXiv:0711.2451] [inSPIRE].

[76] K.A. Intriligator and N. Seiberg, The Runaway quiver, JHEP 02 (2006) 031 [hep-th/0512347] [INSPIRE].

[77] S. Krippendorf, M.J. Dolan, A. Maharana and F. Quevedo, D-branes at Toric Singularities: Model Building, Yukawa Couplings and Flavour Physics, JHEP 06 (2010) 092 [arXiv: 1002.1790] [INSPIRE].

[78] M.J. Dolan, S. Krippendorf and F. Quevedo, Towards a Systematic Construction of Realistic D-brane Models on a del Pezzo Singularity, JHEP 10 (2011) 024 [arXiv:1106.6039] [INSPIRE].

[79] J.J. Heckman, C. Vafa, H. Verlinde and M. Wijnholt, Cascading to the MSSM, JHEP 06 (2008) 016 [arXiv: 0711.0387] [INSPIRE].

[80] M. Yamazaki, Brane Tilings and Their Applications, Fortsch. Phys. 56 (2008) 555 [arXiv:0803.4474] [INSPIRE].

[81] R. Argurio et al., Dimers, Orientifolds and Anomalies, JHEP 02 (2021) 153 [arXiv: 2009.11291] [INSPIRE].

[82] M. Wijnholt, Parameter space of quiver gauge theories, Adv. Theor. Math. Phys. 12 (2008) 711 [hep-th/0512122] [INSPIRE].

[83] I. García-Etxebarria, F. Quevedo and R. Valandro, Global String Embeddings for the Nilpotent Goldstino, JHEP 02 (2016) 148 [arXiv:1512.06926] [INSPIRE].

[84] S. AbdusSalam, S. Abel, M. Cicoli, F. Quevedo and P. Shukla, A systematic approach to Kähler moduli stabilisation, JHEP 08 (2020) 047 [arXiv:2005.11329] [INSPIRE].

[85] M. Cicoli, D. Ciupke, C. Mayrhofer and P. Shukla, A Geometrical Upper Bound on the Inflaton Range, JHEP 05 (2018) 001 [arXiv: 1801.05434] [INSPIRE].

[86] R. Altman, J. Gray, Y.-H. He, V. Jejjala and B.D. Nelson, A Calabi-Yau Database: Threefolds Constructed from the Kreuzer-Skarke List, JHEP 02 (2015) 158 [arXiv: 1411.1418] [INSPIRE]. 
[87] M. Demirtas, L. McAllister and A. Rios-Tascon, Bounding the Kreuzer-Skarke Landscape, arXiv: 2008.01730 [INSPIRE].

[88] R. Blumenhagen, B. Jurke, T. Rahn and H. Roschy, Cohomology of Line Bundles: A Computational Algorithm, J. Math. Phys. 51 (2010) 103525 [arXiv:1003.5217] [INSPIRE].

[89] R. Blumenhagen, B. Jurke and T. Rahn, Computational Tools for Cohomology of Toric Varieties, Adv. High Energy Phys. 2011 (2011) 152749 [arXiv:1104.1187] [INSPIRE].

[90] A. Collinucci and R. Savelli, On Flux Quantization in F-theory, JHEP 02 (2012) 015 [arXiv: 1011.6388] [INSPIRE].

[91] P. Berglund and I. Garcia-Etxebarria, D-brane instantons on non-Spin cycles, JHEP 01 (2013) 056 [arXiv: 1210.1221] [InSPIRE].

[92] S.H. Katz and E. Sharpe, D-branes, open string vertex operators, and Ext groups, Adv. Theor. Math. Phys. 6 (2003) 979 [hep-th/0208104] [INSPIRE].

[93] T. Gomez and E.R. Sharpe, D-branes and scheme theory, hep-th/0008150 [INSPIRE].

[94] A. Collinucci, F. Denef and M. Esole, D-brane Deconstructions in IIB Orientifolds, JHEP 02 (2009) 005 [arXiv: 0805.1573] [inSPIRE].

[95] F. Marchesano, R. Savelli and S. Schwieger, Compact T-branes, JHEP 09 (2017) 132 [arXiv: 1707.03797] [INSPIRE].

[96] F. Marchesano, R. Moraru and R. Savelli, A vanishing theorem for T-branes, JHEP 11 (2020) 002 [arXiv: 2007.02960] [InSPIRE].

[97] A. Collinucci and R. Savelli, T-branes as branes within branes, JHEP 09 (2015) 161 [arXiv: 1410.4178] [INSPIRE].

[98] J.P. Conlon, F. Quevedo and K. Suruliz, Large-volume flux compactifications: Moduli spectrum and D3/D7 soft supersymmetry breaking, JHEP 08 (2005) 007 [hep-th/0505076] [INSPIRE].

[99] M. Cicoli, J.P. Conlon and F. Quevedo, General Analysis of LARGE Volume Scenarios with String Loop Moduli Stabilisation, JHEP 10 (2008) 105 [arXiv:0805.1029] [INSPIRE].

[100] S. Gukov, C. Vafa and E. Witten, CFT's from Calabi-Yau four folds, Nucl. Phys. B 584 (2000) 69 [Erratum ibid. 608 (2001) 477] [hep-th/9906070] [INSPIRE].

[101] K. Becker, M. Becker, M. Haack and J. Louis, Supersymmetry breaking and alpha-prime corrections to flux induced potentials, JHEP 06 (2002) 060 [hep-th/0204254] [INSPIRE].

[102] J.P. Conlon, D. Cremades and F. Quevedo, Kähler potentials of chiral matter fields for Calabi-Yau string compactifications, JHEP 01 (2007) 022 [hep-th/0609180] [INSPIRE].

[103] L. Aparicio, D.G. Cerdeno and L.E. Ibáñez, Modulus-dominated SUSY-breaking soft terms in F-theory and their test at LHC, JHEP 07 (2008) 099 [arXiv:0805.2943] [INSPIRE].

[104] G. von Gersdorff and A. Hebecker, Kähler corrections for the volume modulus of flux compactifications, Phys. Lett. B 624 (2005) 270 [hep-th/0507131] [INSPIRE].

[105] M. Cicoli, J.P. Conlon and F. Quevedo, Systematics of String Loop Corrections in Type IIB Calabi-Yau Flux Compactifications, JHEP 01 (2008) 052 [arXiv:0708.1873] [INSPIRE].

[106] M. Berg, M. Haack and B. Körs, String loop corrections to Kähler potentials in orientifolds, JHEP 11 (2005) 030 [hep-th/0508043] [INSPIRE]. 
[107] M. Berg, M. Haack and E. Pajer, Jumping Through Loops: On Soft Terms from Large Volume Compactifications, JHEP 09 (2007) 031 [arXiv:0704.0737] [INSPIRE].

[108] D. Ciupke, J. Louis and A. Westphal, Higher-Derivative Supergravity and Moduli Stabilization, JHEP 10 (2015) 094 [arXiv:1505.03092] [INSPIRE].

[109] M. Cicoli, F. Quevedo, R. Savelli, A. Schachner and R. Valandro, Systematics of the $\alpha^{\prime}$ Expansion in F-theory, arXiv:2106.04592 [INSPIRE].

[110] F. Marchesano, D. Prieto and M. Wiesner, F-theory flux vacua at large complex structure, arXiv:2105.09326 [INSPIRE].

[111] S.B. Giddings, S. Kachru and J. Polchinski, Hierarchies from fluxes in string compactifications, Phys. Rev. D 66 (2002) 106006 [hep-th/0105097] [INSPIRE].

[112] M. Demirtas, M. Kim, L. Mcallister and J. Moritz, Vacua with Small Flux Superpotential, Phys. Rev. Lett. 124 (2020) 211603 [arXiv:1912.10047] [INSPIRE].

[113] R. Minasian, T.G. Pugh and R. Savelli, F-theory at order $\alpha^{\prime 3}$, JHEP 10 (2015) 050 [arXiv: 1506. 06756] [INSPIRE].

[114] A. Cole, A. Schachner and G. Shiu, Searching the Landscape of Flux Vacua with Genetic Algorithms, JHEP 11 (2019) 045 [arXiv: 1907.10072] [INSPIRE].

[115] M. Cicoli, C. Mayrhofer and R. Valandro, Moduli Stabilisation for Chiral Global Models, JHEP 02 (2012) 062 [arXiv:1110.3333] [INSPIRE].

[116] R. Blumenhagen, J.P. Conlon, S. Krippendorf, S. Moster and F. Quevedo, SUSY Breaking in Local String/F-Theory Models, JHEP 09 (2009) 007 [arXiv: 0906.3297] [InSPIRE].

[117] L. Aparicio, M. Cicoli, S. Krippendorf, A. Maharana, F. Muia and F. Quevedo, Sequestered de Sitter String Scenarios: Soft-terms, JHEP 11 (2014) 071 [arXiv:1409.1931] [InSPIRE].

[118] J.P. Conlon and E. Palti, Gauge Threshold Corrections for Local Orientifolds, JHEP 09 (2009) 019 [arXiv: 0906.1920] [INSPIRE].

[119] J.P. Conlon and E. Palti, On Gauge Threshold Corrections for Local IIB/F-theory GUTs, Phys. Rev. D 80 (2009) 106004 [arXiv:0907.1362] [INSPIRE].

[120] R. Kallosh and A.D. Linde, Landscape, the scale of SUSY breaking, and inflation, JHEP 12 (2004) 004 [hep-th/0411011] [INSPIRE].

[121] N. Barnaby, J.R. Bond, Z. Huang and L. Kofman, Preheating After Modular Inflation, JCAP 12 (2009) 021 [arXiv:0909.0503] [INSPIRE].

[122] S. Krippendorf, F. Muia and F. Quevedo, Moduli Stars, JHEP 08 (2018) 070 [arXiv: 1806.04690] [INSPIRE].

[123] M. Cicoli, K. Dutta, A. Maharana and F. Quevedo, Moduli Vacuum Misalignment and Precise Predictions in String Inflation, JCAP 08 (2016) 006 [arXiv: 1604.08512] [INSPIRE].

[124] M. Demirtas, M. Kim, L. McAllister and J. Moritz, Conifold Vacua with Small Flux Superpotential, Fortsch. Phys. 68 (2020) 2000085 [arXiv:2009.03312] [INSPIRE].

[125] R. Álvarez-García, R. Blumenhagen, M. Brinkmann and L. Schlechter, Small Flux Superpotentials for Type IIB Flux Vacua Close to a Conifold, arXiv:2009.03325 [INSPIRE].

[126] H. Ooguri, E. Palti, G. Shiu and C. Vafa, Distance and de Sitter Conjectures on the Swampland, Phys. Lett. B 788 (2019) 180 [arXiv:1810.05506] [INSPIRE]. 
[127] K.S. Babu and R.N. Mohapatra, Minimal Supersymmetric Left-Right Model, Phys. Lett. B 668 (2008) 404 [arXiv:0807.0481] [INSPIRE].

[128] S. Franco, A. Hanany, D. Krefl, J. Park, A.M. Uranga and D. Vegh, Dimers and orientifolds, JHEP 09 (2007) 075 [arXiv:0707.0298] [INSPIRE].

[129] A. Collinucci and I. García-Etxebarria, $E_{6}$ Yukawa couplings in F-theory as D-brane instanton effects, JHEP 03 (2017) 155 [arXiv: 1612.06874] [INSPIRE].

[130] A. Maleknejad, Axion Inflation with an $\mathrm{SU}(2)$ Gauge Field: Detectable Chiral Gravity Waves, JHEP 07 (2016) 104 [arXiv: 1604.03327] [INSPIRE].

[131] A. Maleknejad, $\mathrm{SU}(2)_{R}$ and its Axion in Cosmology: A common Origin for Inflation, Cold Sterile Neutrinos, and Baryogenesis, arXiv:2012.11516 [INSPIRE].

[132] A. Maleknejad, Chiral Anomaly in $\mathrm{SU}(2)_{R}$-Axion Inflation and the New Prediction for Particle Cosmology, arXiv:2103.14611 [INSPIRE].

[133] I. García-Etxebarria, B. Heidenreich and T. Wrase, New $N=1$ dualities from orientifold transitions - Part II: String Theory, JHEP 10 (2013) 006 [arXiv:1307.1701] [INSPIRE].

[134] M. Cicoli, D. Ciupke, V.A. Diaz, V. Guidetti, F. Muia and P. Shukla, Chiral Global Embedding of Fibre Inflation Models, JHEP 11 (2017) 207 [arXiv: 1709.01518] [INSPIRE].

[135] X. Gao and P. Shukla, On Classifying the Divisor Involutions in Calabi-Yau Threefolds, JHEP 11 (2013) 170 [arXiv:1307.1139] [INSPIRE]. 